\title{
Verzeichnis der Mitglieder der Vereinigung der Deutschen Staatsrechtslehrer e.V.
}

(Stand: 11. Januar 2012; ständige Aktualisierung unter www.staatsrechtslehrer.de)

Vorstand

1. Wieland, Dr. Joachim, LL.M., Universitätsprofessor, Gregor-Mendel-Straße 13, 53115 Bonn, (02 28) 923993 34, Fax (02 28) 32948 98;

Lehrstuhl für öffentliches Recht, Finanz- und Steuerrecht, Deutsche Hochschule für Verwaltungswissenschaften Speyer, Postfach 1409, 67324 Speyer, (0 62 32) 6543 55, Fax (0 62 32) 6543 06, E-Mailwieland@dhv-speyer.de

2. Biaggini, Dr. Giovanni, o. Professor, Kantstraße 12, CH-8044 Zürich, (00 41) 442511158 ;

Lehrstuhl für Staats-, Verwaltungs- und Europarecht, Rechtswissenschaftliches Institut, Freiestrasse 15, CH-8032 Zürich, (00 41) 446 34-30 11 oder -36 68, Fax (00 41) 446 34-43 89, E-Mail lst.biaggini@rwi.unizh.ch

3. Ruffert, Dr. Matthias, Professor, Naumannstraße 12, 07743 Jena, (0 36 41) 2072 63;

Rechtswissenschaftliche Fakultät, Universität Jena, Carl-Zeiss-Straße 3, 07743 Jena, (0 36 41) 9422 01, Fax (0 36 41) 9422 02, E-Mail m.ruffert@recht.uni-jena.de 
Mitglieder:

1. Adamovich, Dr. Dr. h.c. mult. Ludwig, o. Univ.-Prof., Präsident des Österreichischen Verfassungsgerichtshofs a.D., Rooseveltplatz 4, A-1090 Wien, (00 43) 66424275 26;

Österreichische Präsidentschaftskanzlei, Hofburg, Ballhausplatz, A-1014 Wien, (00 43) 1534 22-3 00, Fax (00 43) 1534 22-2 48, E-Mail ludwig.adamovich@hofburg.at

2. Albers, Dr. iur., Dipl. soz. Marion, Professorin, Sulzer Straße 21a, 86159 Augsburg; Universität Hamburg, Fakultät für Rechtswissenschaften, Lehrstuhl für Öffentliches Recht, Informationsund Kommunikationsrecht, Rechtstheorie, Rothenbaumchaussee 33, 20148 Hamburg, (040) 42838 - 57 52, Fax (040) 42838 - 2635 , E-Mail marion.albers@jura.uni-hamburg.de

3. Alexy, Dr. Dr. h.c. mult. Robert, o. Professor, Klausbrooker Weg 122, 24106 Kiel, (04 31) 5497 42;

Universität Kiel, 24098 Kiel, (04 31) 88035 43, Fax (04 31) 88037 45, E-Mail alexy@law.uni-kiel.de

4. Alleweldt, Dr. Ralf, LL.M., Privatdozent, Halbe Stadt 12, 15230 Frankfurt (Oder), (03 35) 622 47;

Europa-Universität Viadrina, Postfach 1786, 15207 Frankfurt (Oder), (03 35) 55347875 ,

E-Mail alleweld@euv-ffo.de

5. Anderheiden, Dr. Michael, Privatdozent, Stephanienstr. 32, 76133 Karlsruhe, (07 21) 47008 17;

Ruprecht-Karls-Universität Heidelberg, Juristisches Seminar, Friedrich-Ebert-Anlage 6-10, 69117 Heidelberg, (0 62 21) 5474 97, Fax (0 62 21) 5474 63, E-Mail anderheidenm@jurs.uni-heidelberg.de 
6. Appel, Dr. Ivo, Professor, Eisvogelweg 28, 82140 Olching, (0 81 42) 28423 17;

Universität Augsburg, Juristische Fakultät, 86135 Augsburg, (08 21) 59845 35, Fax (08 21) 59845 37, E-Mail Ivo.Appel@jura.uni-augsburg.de

7. Arnauld, Dr. Andreas von, Professor, Lange Reihe 103, 20099 Hamburg, (0 40) 318174 17, E-Mail andreas.arnauld@gmx.de; Helmut-Schmidt-Universität/Universität der Bundeswehr Hamburg, Fakultät für Wirtschafts- und Sozialwissenschaften, Professur für Öffentliches Recht, insb. Völker- und Europarecht, Holstenhofweg 85, 22043 Hamburg, (0 40) 65 41-27 71, Fax (0 40) 65 41-20 21, E-Mail arnauld@hsu-hh.de

8. Arnim, Dr. Hans Herbert von, o. Professor, Im Oberkämmerer 26, 67346 Speyer, (0 62 32) 981 23;

Deutsche Hochschule für Verwaltungswissenschaften Speyer, 67324 Speyer, (0 62 32) 654343 , E-Mail vonarnim@dhv-speyer.de

9. Arnold, Dr. Rainer, o. Professor, Plattenweg 7, 93055 Regensburg, (09 41) 744 65;

Universität Regensburg, 93053 Regensburg, (09 41) 9 43-26 54/5, E-Mail Rainer.Arnold@jura.uni-regensburg.de

10. Aschke, Dr. Manfred, Professor, Kantstr. 14, 99425 Weimar, (0 36 43) 4022 83, Fax (0 36 43) 4022 84;

E-Mail winckelmann.aschke@t-online.de; c/o Professur Öffentliches Recht II, Hein-Heckroth-Str. 5, 35390 Gießen oder Thüringer Oberverwaltungsgericht Kaufstr. 2-4, 99423 Weimar, (0 36 43) 2 06-2 69, 
11. Augsberg, Dr. Steffen, Professor, Semperstr. 20, 22303 Hamburg;

Universität des Saarlandes,

Rechts-. und Wirtschaftswissenschaftliche Fakultät,

Lehrstuhl für Öffentliches Recht, insb. Recht des Gesundheitswesens,

Campus Gebäude B 4.4, 66123 Saarbrücken, (0178) 8314000 ,

E-Mail steffen.augsberg@uni-koeln.de

12. Aulehner, Dr. Josef, Privatdozent, Hans-Böcker-Str. 8, 80995 München, (0 89) 12384 02, Fax (0 89) 127496 88; Ludwig-Maximilians-Universität München, Ref. I A 3 - Rechtsabteilung, Geschwister-Scholl-Platz 1, 80539 München, (0 89) 21 80-37 30, Fax (0 89) 21 80-29 85, E-Mail Aulehner@lmu.de

13. Axer, Dr. Peter, Professor, Ruprecht-Karls-Universität Heidelberg, Lehrstuhl für Sozialrecht in Verbindung mit dem Öffentlichen Recht, Friedrich-Ebert-Anlage 6-10, 69117 Heidelberg, (0 62 21) 54-77 68, Fax (0 62 21) 54-77 69, E-Mail axer@jurs.uni-heidelberg.de

14. Baade, Dr. Hans W., Professor, 6002 Mountain Climb Drive, Austin/Texas, USA, 78 731, (0 01-5 12) 4525071 ; dienstl., (0 01-5 12) 47151 51, E-Mail hbaade@mail.law.utexas.edu

15. Badura, Dr. Peter, o. Professor, Am Rothenberg Süd 4, 82431 Kochel am See, (0 88 51) 5289 ;

Universität München, Professor-Huber-Platz 2, 80539 München, (0 89) 21 80-35 76 
16. Baer, Dr. Susanne, LL.M., Professorin, Richterin des Bundesverfassungerichts, Bleibtreustrasse 55, 10623 Berlin;

Humboldt-Universität zu Berlin, Juristische Fakultät, Unter den Linden 9, 10099 Berlin, (0 30) 209334 67, Fax (0 30) 209334 31, E-Mail sekretariat.baer@rewi.hu-berlin.de

17. Baldus, Dr. Manfred, Universitätsprofessor, Herderstr. 41 A, 99096 Erfurt, (03 61) 55470 54;

Lehrstuhl für Öffentliches Recht und Neuere Rechtsgeschichte, Staatswissenschaftliche Fakultät, Universität Erfurt, Nordhäuserstr. 63, 99089 Erfurt, (03 61) 73747 11, E-Mail manfred.baldus@uni-erfurt.de

18. Barfu $\beta$, Dr. iur. Dr. rer. pol. Walter, o. Universitätsprofessor, Tuchlauben 11/31; 1010 Wien;

Präsident des Österreichischen Normungsinstituts, Generaldirektor für Wettbewerb a.D.

(Bundeswettbewerbsbehörde), Heinestraße 38, A-1020 Wien, (00 43) 1/213 00/612, Fax (00 43) 1/213 00/609, E-Mail walter.barfuss@as-institute.at

19. Bartlsperger, Dr. Richard, o. Professor, Schleifweg 55, 91080 Uttenreuth, (0 91 31) 599 16, Fax (0 91 31) 5333 04, E-Mail Bartlsperger.richard@t-online.de

20. Bast, Dr. Jürgen, Privatdozent, Humboldt-Universität zu Berlin, Professur i.V. für Öffentliches Recht, Völker- und Europarecht, Unter den Linden 6, 10099 Berlin, (030) 2093 3512, Fax (030) 2093 3384, E-Mail juergen.bast@rewi.hu-berlin.de 
21. Battis, Dr. Dr. h.c. Ulrich, Professor, Beiersdorfer Weg 42, 12589 Berlin-Rahnsdorf, (0 30) 64819 47;

Humboldt-Universität zu Berlin, Lehrstuhl für Staatsund Verwaltungsrecht sowie Verwaltungswissenschaften, Unter den Linden 6, 10099 Berlin, (0 30) 20 93-35 33, Fax (0 30) 20 93-36 89, E-Mail sekretariat.battis@rewi.hu-berlin.de

22. Bauer, Dr. Hartmut, Professor, Am Hegereiter 13, 01156 Cossebaude, (03 51) 45216 03;

Lehrstuhl für Europäisches und Deutsches Verfassungsrecht, Verwaltungsrecht, Sozialrecht und Öffentliches Wirtschaftsrecht, Universität Potsdam, August-Bebel-Straße 89, 14482 Potsdam, (03 31) 9 77-32 64, Fax (03 31) 9 77-33 10, E-Mail hbauer@rz.uni-potsdam.de

23. Baumeister, Dr. Peter, Professor, Langebrücker Str. 24, 68809 Neulußheim, (0 62 05) 3978 17;

SRH Hochschule Heidelberg, Ludwig-Guttmann-Str. 6, 69123 Heidelberg, (0 62 21) 8822 60, Fax (0 62 21) 8834 82, E-Mail peter.baumeister@fh-heidelberg.de; Schlatter Rechtsanwälte, Kurfürsten-Anlage 59, 69115 Heidelberg, (0 62 21) 9812 17, Fax (0 62 21) 1824 75, E-Mail p.baumeister@kanzlei-schlatter.de

24. Baumgartner, Dr. Gerhard, Univ. Prof., Institut für Rechtswissenschaft, Alpen-Adria-Universität Klagenfurt, Universitätsstr. 65-67, A-9020 Klagenfurt am Wörthersee, +43 4632700 3311, Fax +43 4632700993311 , E-Mail Gerhard.Baumgartner@aau.at

25. Bausback, Dr. Winfried, Univ.-Prof. a.D., MdL, Im Neurod 8, 63741 Aschaffenburg, (0 60 21) 4566 06, Fax (0 60 21) 4566 07; Büro: Roßmarkt 34, 63739 Aschaffenburg, (06021) 4423 20, Fax (06021) 4423 18;

E-Mail info@winfried-bausback.de 
26. Bayer, Dr. Hermann-Wilfried, Professor, Henkenbergstr. 45a, 44797 Bochum, (02 34) 7917 44;

Universität Bochum, 44780 Bochum, (02 34) 32-2 5724

27. Beaucamp, Dr. Guy, Professor, Nordstr. 21, 18107 Elmenhorst, (03 81) 76869 50;

Department Public Management, Fakultät Wirtschaft und Soziales, HAW Hamburg, Berliner Tor 5, 20099 Hamburg, E-Mail AnkeBeauc@aol.com

28. Becker, Dr. Florian, LL.M.(Cambridge), Professor, Universität Kiel, Olshausenstr. 75, Gebäude II, (Postanschrift: Olshausenstr. 40), 24098 Kiel, (04 31) 8 80-53 78 oder (04 31) 8 80-15 04, Fax (04 31) 8 80-53 74, E-Mail f.becker@law.uni-kiel.de

29. Becker, Dr. Joachim, Privatdozent, Kreuznacher Str. 6, 14197 Berlin, (0 30) 82240 12;

Humboldt-Universität zu Berlin, Juristische Fakultät, Unter den Linden 6, 10099 Berlin, (0 30) 20933383 , E-Mail Joachim.Becker@rewi.hu-berlin.de

30. Becker, Dr. Jürgen, o. Professor, Kellerstr. 7, 81667 München;

Stellvertretender Vorsitzender des Vorstands und Chefsyndikus der GEMA, Rosenheimer Straße 11, 81667 München, (0 89) 480 03-00, Fax (0 89) 480 03-6 20 E-Mail jbecker@gema.de

31. Becker, Dr. Ulrich, LL.M. (EHI), Professor, Pfarrsiedlungsstr. 9, 93161 Sinzing, (0 94 04) 34 78;

Max-Planck-Institut für ausländisches und internationales Sozialrecht, Amalienstr. 33, 80799 München, (0 89) 386 02-5 11, Fax (0 89) 386 02-5 90, E-Mail Becker@mpisoc.mpg.de 
32. Belser, Dr. Eva Maria, Professorin, Chemin du Riedelet 7, CH-1723 Marly, (00 41) 2643622 36;

Universität Freiburg i. Ue., Lehrstuhl für Staats- und Verwaltungsrecht, Institut für Föderalismus, Route d'Englisberg 7, CH-1763 Granges-Paccot, (00 41) 2630081 30, evamaria.belser@unifr.ch

33. Berchtold, Dr. Klaus, Universitätsprofessor, Bräunerstr. 4-6/22, A-1010 Wien, (00 43) 1531434

34. Berg, Dr. Wilfried, o. Professor, Waldsteinring 25, 95448 Bayreuth, (09 21) 99008 14; Universität Bayreuth, 95440 Bayreuth, (09 21) 5528 76, Fax (09 21) 55842875 oder 552985 , E-Mail wilfried@cwberg.de

35. Berka, Dr. Walter, o. Universitätsprofessor, Birkenweg 2, A-5400 Hallein, (00 43) 66245767 58;

Institut für Verfassungs- und Verwaltungsrecht der Universität Salzburg, Kapitelgasse 5-7, A-5020 Salzburg, (00 43) 6 62-80 4436 21, Fax (00 43) 6 62-80 4436 29, E-Mail Walter.Berka@sbg.ac.at

36. Bernhardt, Dr. Dr. h.c. Rudolf, o. Professor, Gustav-Kirchhoff-Str. 2a, 69120 Heidelberg, (0 62 21) 4136 99;

MPI für ausländisches öffentliches Recht und Völkerrecht, Im Neuenheimer Feld 535, 69120 Heidelberg, (0 62 21) 4822 53, E-Mail rbernhar@mpil.de

37. Bernstorff, Dr. Jochen von, Professor, Eberhard-Karls-Universität Tübingen, Juristische Fakultät, Lehrstuhl für Staatsrecht, Völkerrecht und Verfassungslehre, Geschwister-Scholl-Platz, 72074 Tübingen, E-Mail jvbernst@mpil.de 
38. Bethge, Dr. Herbert, o. Professor, Am Seidenhof 8, 94034 Passau, (08 51) 416 97, Fax (08 51) 49018 97, E-Mail H.I.Bethge@t-online.de

39. Beyerlin, Dr. Ulrich, apl. Professor, Luisenstr. 7, 69151 Neckargmünd; MPI für ausländisches öffentliches Recht und Völkerrecht, Im Neuenheimer Feld 535, 69120 Heidelberg, (0 62 21) 4822 30, E-Mail ubeyerli@mpil.de

40. Biaggini, Dr. Giovanni, o. Professor, Kantstraße 12, CH-8044 Zürich, (00 41) 442511158 ;

Lehrstuhl für Staats-, Verwaltungs- und Europarecht, Rechtswissenschaftliches Institut, Freiestrasse 15, CH-8032 Zürich, (00 41) 446 34-30 11 oder -36 68, Fax (00 41) 446 34-43 89, E-Mail lst.biaggini@rwi.unizh.ch

41. Bieber, Dr. Uwe Roland, o. Professor, Mainzer Str. 135, 53179 Bonn, (02 28) 357189 ;

Université de Lausanne, Faculté de Droit - CDCE BFSH 1, CH-1015 Lausanne-Dorigny, (00 41) 21-6 9227 90, Fax (00 41) 21-6 9227 85, E-Mail Roland.Bieber@unil.ch

42. Binder, Dr. Bruno, Universitätsprofessor, Wischerstr. 30, A-4040 Linz, (00 43) 7 32-71 77 72-0, Fax (00 43) 7 32-71 77 72-4;

Universität Linz, Altenbergerstr. 69, A-4020 Linz, (00 43) 732246 80, Fax (00 43) 7 32-24 68 10,

E-Mail vwrecht@jku.at

43. Birk, Dr. Dieter, o. Professor, Borkumweg 43, 48159 Münster, (02 51) 2184 78, Fax (02 51) 2184 76;

Universität Münster, 48143 Münster, (02 51) 83227 95, Fax (02 51) 83283 86, E-Mail Birk@uni-muenster.de 
44. Blanke, Dr. Hermann-Josef, Universitätsprofessor, Lehrstuhl für Öffentliches Recht, Völkerrecht und Europäische Integration, Universität Erfurt, Nordhäuser Straße 63, 99089 Erfurt, (03 61) 7 37-47 51, (03 61) 7 37-47 00 (Sekr.), Fax (03 61) 7 37-47 09, E-Mail LS_Staatsrecht@uni-erfurt.de

45. Blankenagel, Dr. Alexander, Professor, Türksteinstraße 10, 14167 Berlin, (0 30) 85495 82;

Humboldt-Universität zu Berlin, Juristische Fakultät, Unter den Linden 6, 10099 Berlin, (0 30) 20 93-33 81, Fax (0 30) 20 93-33 45, E-Mail blankenagel@rewi.hu-berlin.de

46. Blümel, Dr. Willi, Universitätsprofessor, Angelhofweg 65, 69259 Wilhelmsfeld, (0 62 20) 18 80;

Deutsche Hochschule für Verwaltungswissenschaften Speyer, 67324 Speyer, (0 62 32) 6 54-3 62 oder -360 , Fax (0 62 32) 9 10-2 08 oder 9 10-2 90

47. Bock, Dr. Wolfgang, Privatdozent, Richter am Landgericht Frankfurt am Main, Schalkwiesenweg 44, 60488 Frankfurt am Main, Privat: (0 69) 7657 17; (0163) 6362 552, Dienstlich: (0611) 720325

E-Mail wolfgang.bock@lfv.hessen.de

48. Böckenförde, Dr. iur. Dr. phil. Dr. h.c. Ernst-Wolfgang, o. Professor, Türkheimstr. 1, 79280 Au bei Freiburg, (07 61) 4056 23;

Albert-Ludwigs-Universität Freiburg, 79098 Freiburg, (07 61) 2032263 oder -2262 
49. Bogdandy, Dr. Armin von, M.A., Professor, Mühltalstr. 117, 69121 Heidelberg, (0 62 21) 5894 33;

Max-Planck-Institut für ausländisches öffentliches Recht und Völkerrecht, Im Neuenheimer Feld 535, 69120 Heidelberg, (0 62 21) 4826 02, Fax (0 62 21) 482603 , E-Mail sekreavb@mpil.de

50. Bogs, Dr. Harald, o. Professor, Anton-Bartl-Str. 4, 82327 Tutzing, (08158) 906583

51. Böhm, Dr. Monika, Professorin, Lerchenweg 7, 65719 Hofheim/Ts., (0 61 92) 248 29, Fax (0 61 92) 248 14;

Philipps-Universität Marburg, Institut für Öffentliches Recht, Savignyhaus, Raum 404, Universitätsstraße 6, 35032 Marburg/Lahn, (0 64 21) 2 82-38 08 oder -38 08, Fax (0 64 21) 2 82-89 82, E-Mail Monika.Boehm@staff.uni-marburg.de

52. Bohne, Dr. Eberhard, M.A., Professor, Conrad-Hist-Straße 35, 67346 Speyer, (0 62 32) 737 04, Fax (0 62 32) 60108 71; Deutsche Hochschule für Verwaltungswissenschaften Speyer, Freiherr-vom-Stein-Straße 2, 67346 Speyer, (0 62 32) 6 54-3 26, Fax (0 62 32) 6 54-4 16, E-Mail bohne@dhv-speyer.de

53. Borowski, Dr. Martin, Privatdozent, Senior Lecturer, 68 High Point, Richmond Hill Road, Edgbaston Birmingham B15 3RS, United Kingdom; University of Birmingham, School of Law, Edgbaston Birmingham B15 2TT, United Kingdom, (00 44) 12141432 33, Fax (00 44) 12141435 85, E-Mailm.borowski@bham.ac.uk

54. Bothe, Dr. Michael, Universitätsprofessor, Theodor-Heuss-Str. 6, 64625 Bensheim, (0 62 51) 43 45;

Universität Frankfurt am Main, Juridicum Zimmer 210, Senckenberganlage 31, 60054 Frankfurt am Main, (0 69) 79822264 , E-Mail bothe-bensheim@t-online.de 
55. Brandt, Dr. Edmund, Professor,

Technische Universität Braunschweig,

Carl-Friedrich-Gauß-Fakultät,

Institut für Rechtswissenschaften,

Lehrstuhl Staats- und Verwaltungsrecht sowie

Verwaltungswissenschaften,

Bienroder Weg 87, 38106 Braunschweig,

(05 31) 3 91-24 61, Fax (05 31) 3 91-24 66

E-Mail edmund.brandt@tu-bs.de

56. Breitenmoser, Dr. Stephan, Professor,

Ordinarius für Europarecht,

Juristische Fakultät der Universität Basel,

Peter Merian-Weg 8, Postfach, CH-4002 Basel,

(00 41) 6126725 51, Fax (00 41) 6126725 79,

E-Mail stephan.breitenmoser@unibas.ch

57. Brenner, Dr. Michael, Professor, Adlerstraße 29, 73550 Waldstetten, (0 71 71) 996742 Fax (0 71 71) 9968 65;

Lehrstuhl für Deutsches und Europäisches Verfassungsund Verwaltungsrecht, Universität Jena, Carl-Zeiss-Str. 3, 07743 Jena, (0 36 41) 942240 oder -41, Fax (0 36 41) 9422 42, E-Mail Prof.Brenner@t-online.de

58. Breuer, Dr. Marten, Privatdozent, Claussen-Simon-Dozent für Europäisches und Internationales Recht, Europa-Kolleg Hamburg

Windmühlenweg 27

22607 Hamburg

(040) 822727 26, Fax (040) 82272798

m-breuer@europa-kolleg-hamburg.de

59. Breuer, Dr. Rüdiger, Professor,

Buschstr. 56, 53113 Bonn,

(02 28) 2179 72, Fax (02 28) 2248 32;

Köhler \& Klett Rechtsanwälte, Köln, (02 21) 42 07-2 91, Fax (02 21) 42 07-2 55,

E-Mail breuer.ruediger@arcor.de 
60. Brinktrine, Dr. Ralf, Universitätsprofessor, Margaretenstr. 31, 97276 Margetshöchheim, (0931) 304458 84,

Lehrstuhl für Öffentliches Recht, deutsches und

europäisches Umweltrecht und Rechtsvergleichung,

Juristische Fakultät, Universität Würzburg,

Domerschulstraße 16, 97070 Würzburg,

(09 31) 3 18-23 31,

E-Mail Ralf.Brinktrine@jura.uni-wuerzburg.de

61. Britz, Dr. Gabriele, Professorin,

Richterin des Bundesverfassungsgerichts,

Justus-Liebig-Universität Gießen,

Professur für Öffentliches Recht und Europarecht,

Hein-Heckroth-Straße 5, 35390 Gießen, (06 41) 9921070 Fax (06 41) 99210 79,

E-Mail Gabriele.Britz@recht.uni-giessen.de

62. Brohm, Dr. Winfried, o. Professor,

Wydenmööslistr. 11, CH-8280 Kreuzlingen,

(00 41) 71-6 8815 25;

Universität Konstanz,

Postfach 5560 D 100, 78434 Konstanz,

(0 7531$) 882169$ oder -2176

63. Bröhmer, Dr. Jürgen, Professor,

11 Kennedy Street, 2350 Armidale,

(00 61) 2-67 72-46 47;

Head of School, School of Law, University of New England, Armidale, NSW, 2301, Australien, (00 61) 2-67 73-35 98,

E-Mail jurgen.brohmer@une.edu.au

64. Brosius-Gersdorf, Dr. Frauke, LL.M., Professorin, Alte Leipziger Str. 10, 10117 Berlin, (030) 206196 59, Fax (030) 206196 62;

Gottfried Wilhelm Leibniz Universität Hannover, Lehrstuhl für Öffentliches Recht, insb. Sozialrecht, Öffentliches Wirtschaftsrecht und Verwaltungswissenschaft,

Königsworther Platz 1, 30167 Hannover, (0511) 762-8225, Mobil (0173) 60914 54, Fax (0511) 762-8228;

E-Mail brosius-gersdorf@jura.uni-hannover.de 
65. Brühl-Moser, Dr. Denise, Privatdozentin, Unt. Batterieweg 167, CH-4059 Basel, (00 41) 765581042 ,

E-Mail d.bruehl-moser@unibas.ch

66. Brüning, Dr. Christoph, Professor, Kiebitzredder 12, 24220 Flintbek, (0 43 47) 71342 95;

Universität Kiel, Olshausenstr. 75, 24118 Kiel, (04 31) 8 80-45 40 oder -15 05, Fax (04 31) 8 80-45 82, E-Mail cbruening@law.uni-kiel.de

67. Brünneck, Dr. Alexander von, Professor, Blumenhagenstr. 5, 30167 Hannover, (05 11) 7169 11;

Europa-Universität Viadrina, 15207 Frankfurt (Oder), Postfach 17 86, (03 35) 55 34-22 64 oder -22 95, Fax (03 35) 55 34-24 18, E-Mail raue@euv-frankfurt-o.de

68. Bryde, Dr. Brun-Otto, o. Professor, Richter des Bundesverfassungsgerichts a.D., Universität Gießen, Hein-Heckroth-Str. 5, 35390 Gießen, (0 64 1) 99-2 10 60/61, Fax (06 41) 99-2 10 69, E-Mail Brun.O.Bryde@recht.uni-giessen.de

69. Bull, Dr. Hans Peter, o. Professor, Falckweg 16, 22605 Hamburg, (0 40) 8805652 , E-Mail HP-Bull@t-online.de

70. Bullinger, Dr. Dr. h.c. (Université de Dijon), Martin, o. Professor, Altschlößleweg 4, 79280 Au bei Freiburg, (07 61) 4023 89;

Universität Freiburg, 79085 Freiburg, (07 61) 2032248 oder -47 , E-Mail martin.bullinger@jura.uni-freiburg.de 
71. Bultmann, Dr. Peter Friedrich, Privatdozent, Am Pankepark 51, 10115 Berlin, (0 30) 440564 43;

Humboldt-Universität zu Berlin, Unter den Linden 6, 10099 Berlin, E-Mail piet.bultmann@rz.hu-berlin.de

72. Bumke, Dr. Christian, Professor, Apostel-Paulus-Str. 19, 10825 Berlin, (0 30) 78267 87;

Bucerius Law School, Jungiusstraße 6, 20355 Hamburg, (0 40) 307 06-2 37, Fax (0 40) 307 06-2 59, E-Mail christian.bumke@law-school.de

73. Bungenberg, Dr. Marc, LL.M. (Lausanne), Professor, Pirmasenser Str. 3, 30559 Hannover, (0511) 2193413 oder (0177) 43497 22; Universität Siegen, Fachbereich Wirtschaftswissenschaften, Wirtschaftsrecht und Wirtschaftsinformatik, Lehrstuhl für Öffentliches Recht und Europarecht, Hölderlinstr. 3, 57068 Siegen, (0271) 740 3219, Fax (0271) 740 2477, E-Mail marc.bungenberg@gmx.de

74. Burgi, Dr. Martin, Professor, Bernhard-Poether-Str. 59, 48165 Münster, (0 25 01) 9288 93; Lehrstuhl für Öffentliches Recht, Wirtschaftsverfassungs- und Wirtschaftsverwaltungsrecht, Ruhr-Universität Bochum, 44780 Bochum, (02 34) 32252 75, Fax (02 34) 32142 82, E-Mail Martin.Burgi@jura.ruhr-uni-bochum.de

75. Burkert, Dr. Herbert, Professor, Uferstr. 31, 50996 Köln-Rodenkirchen, (00 49) 2213977 00, Fax (00 49) 2213977 11; MCM-HSG, Universität St. Gallen, Müller-Friedberg-Str. 8, CH-9000 St. Gallen, (00 41) 71-2 22 48 75, Fax (00 41) 71-2 2248 75, E-Mail herbert.burkert@unisg.ch 
76. Bußjäger, Dr. Peter, Privatdozent, Institut für Föderalismus, Maria-Theresien-Straße 38b, A-6020 Innsbruck, (00 43) 5 12-57 45 94, Fax (00 43) 5 12-57 45 94-4

77. Butzer, Dr. iur. Hermann, Professor, Moltkestr. 4, 30989 Gehrden, (0 5108$) 8782323$; Leibniz-Universität Hannover, Lehrstuhl für Öffentliches Recht, insbesondere Recht der staatlichen Transfersysteme, Königsworther Platz 1, 30167 Hannover, (05 11) 76281 69, Fax (05 11) 76282 03, E-Mail butzer@jura.uni-hannover.de

78. Calliess, Dr. Christian, LL.M. Eur., M.A.E.S. (Brügge), Professor, (01 75) 20575 22;

Freie Universität Berlin, Lehrstuhl für Öffentliches Recht und Europarecht, Van't-Hoff-Str. 8, 14195 Berlin, (0 30) 83 8-5 14 56, Fax (0 30) 83 8-5 30 12, E-Mail europarecht@fu-berlin.de

79. Cancik, Dr. Pascale, Professorin, Martinistr. 33, 49080 Osnabrück, (05 41) 9 70-19 77;

Universität Osnabrück, Fachbereich Rechtswissenschaften, Martinistraße 8, 49069 Osnabrück, (05 41) 9 69-60 44, (05 41) 9 69-61 68 (Sekr.), E-Mail pcancik@uni-osnabrueck.de

80. Caspar, Dr. Johannes, Privatdozent, Tronjeweg 16, 22559 Hamburg, (0 40) 819611 95, Fax (0 40) 819611 21; Universität Hamburg, Fachbereich Rechtswissenschaft, Edmund-Siemers-Allee 1, Flügel West, 20146 Hamburg, (0 40) 428 38-57 60, Fax (0 40) 428 38-62 80, Der Hamburgische Beauftragte für Datenschutz und Informationsfreiheit, Klosterwall 6 (Block C), 20095 Hamburg, E-Mail johannes.caspar@datenschutz.hamburg.de 
81. Classen, Dr. Claus Dieter, Professor, Olchinger Str. $57 \mathrm{~g}, 82178$ Puchheim, (0 89) 894188 00, Fax (0 89) 894188 01; Ernst-Moritz-Arndt-Universität Greifswald, 17487 Greifswald, (0 38 34) 862121 oder 21 24, Fax (0 38 34) 8620 02, E-Mail Classen@uni-greifswald.de

82. Coelln, Dr. Christian von, Professor, Prinz-Georg-Str. 104, 40479 Düsseldorf, (02 11) 995438 38, E-Mail coelln@web.de; Universität zu Köln, Institut für Deutsches und Europäisches Wissenschaftsrecht, Albert-Magnus-Platz, 50923 Köln, (02 21) 4 70-40 66, Fax (02 21) 4 70-16 92, E-Mail cvcoelln@uni-koeln.de

83. Collin, Dr. Peter, Privatdozent, Rykestr. 18, 10405 Berlin; MPI für europäische Rechtsgeschichte, Hausener Weg 120, 60489 Frankfurt am Main, (0 69) 789 78-1 61, (0 30) 400562 92, Fax (0 69) 789 78-1 69, E-Mail collin@mpier.uni-frankfurt.de

84. Cornils, Dr. Matthias, Professor, Adelheidstr. 92, 65185 Wiesbaden, (06 11) 97199 97, (01 78) 49873 11, E-Mail mcornils@arcor.de;

Johannes Gutenberg-Universität Mainz, Fachbereich Rechts- und Wirtschaftswissenschaften, Jakob-Welder-Weg 9, 55099 Mainz, (0 61 31) 39-2 20 69, E-Mail cornils@uni-mainz.de

85. Cremer, Dr. Hans-Joachim, Universitätsprofessor, Steinritzstr. 21, 60437 Frankfurt am Main; Universität Mannheim, Fakultät für Rechtswissenschaft, Schloß, Westflügel, 68131 Mannheim, (06 21) 1 81-14 28, -14 29 (Sekr.), Fax (06 21) 1 81-14 30, E-Mail Hjcremer@rumms.uni-mannheim.de 
86. Cremer, Dr. Wolfram, Professor, Schellstraße 13, 44789 Bochum;

Ruhr-Universität Bochum, Lehrstuhl für Öffentliches Recht und Europarecht,

GC 8/160,

44780 Bochum,

(02 34) 32-2 28 18, Fax (02 34) 32-1 42 81,

E-Mail wolfram.cremer@rub.de

87. Czybulka, Dr. Detlef, Universitätsprofessor,

Bergstraße 24-25, 18107 Elmenhorst,

(03 81) 79539 44, Fax (03 81) 79539 45;

Universität Rostock, Lehrstuhl für Staats- und Verwaltungsrecht,

Umweltrecht und Öffentliches Wirtschaftsrecht,

Universitätsplatz 1, 18051 Rostock;

(03 81) 4 98-82 50, Fax (03 81) 4 98-82 52,

E-Mail detlef.czybulka@uni-rostock.de

88. Dagtoglou, Dr. Prodromos, Professor,

Hippokratous 33, GR-Athen 144,

(00 30) 132211 90;

dienstl.: (00 30) 13629065

89. Dann, Dr. Philipp, LL.M.,

Privat: Rohrbachstr. 54, 60389 Frankfurt,

(069) 3670 2331;

Dienstlich: Licher Str. 64, 35394 Gießen, (0641) 9921120,

E-Mail pdann@mpil.de

90. Danwitz, Dr. Thomas von, Professor,

Richter am Gerichtshof der Europäischen Gemeinschaften,

Klinkenbergsweg 1, 53332 Bornheim, (0 22 27) 9091 04, Fax (0 22 27) 9091 05;

Institut für Öffentliches Recht und Verwaltungslehre,

Lehrstuhl für Öffentliches Recht und Europarecht,

Wirtschaftsverfassungs- und Wirtschaftsverwaltungsrecht,

(02 21) 4 70-52 80, Fax (02 21) 4 70-51 26,

E-Mail Thomas.vonDanwitz@uni-koeln.de,

Sekretariat: Elke.Huebner@uni-koeln.de;

Gerichtshof der Europäischen Gemeinschaften,

L-2925 Luxemburg,

(0 03 52) 43 03-22 30, Fax (0 03 52) 43 03-20 71,

E-Mail thomas.vondanwitz@curia.europa.eu 
91. Davy, Dr. Benjamin, Universitätsprofessor, Korte Geitke 5, 44227 Dortmund, (02 31) 7799 94;

Technische Universität Dortmund, Fakultät Raumplanung, Lehrstuhl für Bodenpolitik, Bodenmanagement und kommunales Vermessungswesen, August-Schmidt-Str. 10, 44221 Dortmund, (02 31) 75522 28, Fax (02 31) 75548 86, E-Mail benjamin.davy@udo.edu

92. Davy, Dr. Ulrike, Universitätsprofessorin, Korte Geitke 5, 44227 Dortmund, (02 31) 779994 oder 79499 79;

Lehrstuhl für öffentliches Recht, deutsches und internationales Sozialrecht und Rechtsvergleichung, Universität Bielefeld, Postfach 1001 31, 33501 Bielefeld, (05 21) 1064400 oder 6893 (Sekr.), Fax (05 21) 10680 83, E-Mail udavy@uni-bielefeld.de

93. Dederer, Dr. Hans-Georg, Professor, Holländerstr. 13c, 94034 Passau; Universität Passau, Lehrstuhl für Staats- und Verwaltungsrecht, Völkerrecht, Europäisches und Internationales Wirtschaftsrecht, Innstr. 39, 94032 Passau, (08 51) 5 09-23 40, Fax (08 51) 5 09-23 42, E-Mail Hans-Georg.Dederer@uni-passau.de

94. De Wall, Dr. Heinrich, Professor, Schronfeld 108, 91054 Erlangen, (0 91 31) 9715 45;

Hans-Liermann-Institut für Kirchenrecht der Friedrich-Alexander-Universität Erlangen-Nürnberg, Hindenburgstraße 34, 91054 Erlangen, (0 91 31) 85-2 22 42, Fax (0 91 31) 85-2 40 64, E-Mail hli@jura.uni-erlangen.de

95. Degenhart, Dr. Christoph, Universitätsprofessor, Stormstr. 3, 90491 Nürnberg, (09 11) 5924 62, Fax (09 11) 5924 62; Juristenfakultät, Universität Leipzig, Burgstr. 27, 04109 Leipzig, (03 41) 97-3 51 91, Fax (03 41) 97-3 51 99, E-Mail degen@rz.uni-leipzig.de 
96. Delbanco, Dr. Heike, Privatdozentin, Großbeerenstraße 83 A, 28211 Bremen, (04 21) 24363 81, Fax (04 21) 33049 40;

Ärztekammer Bremen,

Schwachhauser Heerstraße 30, 28209 Bremen, (04 21) 34 04-2 00, Fax (04 21) 34 04-2 09, E-Mail delbanco@t-online.de

97. Delbrück, Jost, Dr. Dr. rer.pol.h.c., LL.D.h.c., Professor em., Schoolredder 20, 24161 Altenholz, (04 31) 3239 95;

Universität Kiel, 24098 Kiel, (04 31) 88021 88, Fax (04 31) 88016 19, E-Mail jdelbrueck@web.de

98. Denninger, Dr. Dr. h.c. Erhard, Professor em., Am Wiesenhof 1, 61462 Königstein, (0 61 73) 789 88;

Universität Frankfurt, Institut für Öffentliches Recht, Senckenberganlage 31, 60325 Frankfurt am Main, E-Mail Denninger@jur.uni-frankfurt.de

99. Depenheuer, Dr. Otto, Professor, Joachimstraße 4, 53113 Bonn, (0 22 8) 928943 63, Fax (02 28) 928943 64;

Universität zu Köln, Seminar für Staatsphilosophie und Rechtspolitik, Albertus-Magnus-Platz, 50923 Köln, (02 21) 47022 30, Fax (02 21) 47050 10, E-Mail Depenheuer@uni-koeln.de

100. Desens, Dr. Marc, Universitätsprofessor, Käthe-Kollwitz-Str. 13, 04109 Leipzig, (03 41) 24776 63;

Universität Leipzig, Juristenfakultät, Lehrstuhl für Öffentliches Recht, insb.

Steuerrecht und Öffentliches Wirtschaftsrecht, Otto-Schill-Str. 2, 04109 Leipzig, (03 41) 9735 -270, Fax (0341) 9735 -279 E-Mail marc.desens@uni-leipzig.de 
101. Determann, Dr. Lothar, Privatdozent, 1275 California Street, USA-San Francisco, CA 94109, E-Mail lothar.determann@bakernet.com;

Freie Universität Berlin, Ehrenbergstr. 17, 14195 Berlin

102. Detterbeck, Dr. Steffen, o. Professor, Stettiner Str. 60, 35274 Kirchhain, (0 64 22) 45 31;

Institut für Öffentliches Recht, Universität Marburg,

Savignyhaus, Raum 407, Universitätsstr. 6, 35032 Marburg, (0 64 21) 28231 23, Fax (0 64 21) 28232 09, E-Mail brechta@staff.uni-marburg.de

103. Di Fabio, Dr. Dr. Udo, Professor, Richter des Bundesverfassungsgerichts, Schloßbezirk 3, 76131 Karlsruhe, (07 21) 91 01-0, Fax (07 21) 91 01-3 82; Institut für Öffentliches Recht, Abt. Staatsrecht, Rheinische Friedrich Wilhelms-Universität, Adenauerallee 44, 53113 Bonn, (02 28) 73 55-73, Fax (02 28) 7379 35, E-Maildifabio@uni-bonn.de

104. Dietlein, Dr. Johannes, Professor, Heinrich-Heine-Universität, Lehrstuhl für Öffentliches Recht und Verwaltungslehre, Zentrum für Informationsrecht, Universitätsstr. 1, 40225 Düsseldorf, (02 11) 81-1 14 20, Fax (02 11) 81-1 14 55, E-Mail dietlein@uni-duesseldorf.de

105. Dietz, Dr. Andreas, Privatdozent, Bayerischer Verwaltungsgerichtshof, Postfach 3401 48, 80098 München, (089) 2130-338, E-Mail Andreas.Dietz@vgh.bayern.de 
106. Diggelmann, Dr. Oliver, Professor, Alte Landstrasse 49, 8802 Kilchberg, (00 41) 4324445 35;

Institut für Völkerrecht und ausländisches Verfassungsrecht; Lehrstuhl für Völkerrecht, Europarecht, Öffentliches Recht und Staatsphilosophie;

Rämistrasse 74/36, 8001 Zürich, (0041) 44634 -2054 oder -2033, Fax (0041) $44634-5399$

E-Mail oliver.diggelmann@rwi.uzh.ch

107. Dittmann, Dr. Armin, o. Professor, Karl-Brennenstuhl-Str. 11, 72074 Tübingen, (0 70 71) 824 56;

Universität Hohenheim - Schloß, Postfach 7005 62, 70593 Stuttgart, (07 11) 4 59-27 91, Fax (07 11) 4 59-34 82, E-Mail adittman@uni-hohenheim.de

108. Dolzer, Dr. Dr. Rudolf, Professor, Am Pferchelhang 4/1, 69118 Heidelberg, (0 62 21) 8033 44;

Rechts- und Staatswissenschaftliche Fakultät, Universität Bonn, Adenauerallee 24-42, 53113 Bonn, (02 28) 7391 72, Fax (02 28) 7391 71, E-Mail rdolzer@jura.uni-bonn.de

109. Dörr, Dr. Dieter, Universitätsprofessor, Am Stadtwald 6, 66123 Saarbrücken; Lehrstuhl für Öffentliches Recht, Völker- und Europarecht, Medienrecht, Johannes-Gutenberg-Universität Mainz, 55099 Mainz, (0 61 31) 3922681 oder 39230 44, Fax (0 61 31) 39256 97, E-Mail ddoerr@uni-mainz.de; Mainzer Medieninstitut (MMI): Mainzer Medieninstitut e.V., Jakob-Welder-Weg 4, 55128 Mainz, (0 61 31) 14492 50, Fax (0 61 31) 14492 60, E-Mail doerr@mainzer-medieninstitut.de

110. Dörr, Dr. Oliver, LL.M. (London), Professor, Universität Osnabrück, Fachbereich Rechtswissenschaft, European Legal Studies Institute, 49069 Osnabrück, (05 41) 9696050 oder -60 51, Fax (05 41) 96960 49, E-Mail odoerr@uos.de 
111. Dreier, Dr. Horst, o. Professor, Bismarckstr. 13, 21465 Reinbek, (0 40) 72258 34;

Lehrstuhl für Rechtsphilosophie, Staats- und Verwaltungsrecht, Universität Würzburg, Domerschulstraße 16, 97070 Würzburg, (09 31) 31-8 23 21, Fax (09 31) 31-29 11, E-Mail dreier@mail.uni-wuerzburg.de

112. Dreier, Dr. Ralf, o. Professor, Wilhelm-Weber-Str. 4, 37073 Göttingen, (05 51) 591 14;

Universität Göttingen, 37073 Göttingen, (05 51) 397384

113. Droege, Dr. Michael, Universitätsprofessor, Victor-Achard-Str. 14a, 61350 Bad Homburg v. d. H., (0 6172$) 85694$ 76, Fax (0 32 21) 12956 38;

Universität Osnabrück, Institut für Finanz- und Steuerrecht, Martinistr. 8, 49078 Osnabrück, (05 41) 96961 68, Fax (05 41) 96961 67, E-Mail Michael.Droege@uos.de

114. Drüen, Dr. Klaus-Dieter, Professor, Heinrich-Heine-Universität Düsseldorf, Lehrstuhl für Unternehmenssteuerrecht, Universitätsstraße 1, 40225 Düsseldorf, (02 11) 81-1 58 68, Fax (02 11) 81-1 58 70, E-Mail klaus-dieter.drueen@uni-duesseldorf.de

115. Durner, Dr. jur., Dr. phil. Wolfgang, LL.M. (London), Professor, Viktoriaplatz 1, 53173 Bonn-Bad Godesberg; Rheinische Friedrich-Wilhelms-Universität Bonn, Rechts- und Staatswissenschaftliche Fakultät, Adenauerallee 44, 53113 Bonn, (02 28) 7391 51, Fax (02 28) 7355 82, E-Mail durner@uni-bonn.de 
116. Eberhard, Dr. Harald, Universitätsprofessor, Troststr. 89/16, A-1100 Wien; Wirtschaftsuniversität Wien, Institut für Österreichisches und Europäisches Öffentliches Recht, Althanstraße 39-45, A-1090 Wien, (0043) 131336 -5088, Fax (0043) $131336-713$

E-Mail harald.eberhard@wu.ac.at

117. Eberle, Dr. Carl-Eugen, Professor, Justitiar des ZDF,

Kapellenstr. 68a, 65193 Wiesbaden, (06 11) 520468 ; ZDF, 55100 Mainz, (0 61 31) 70-41 00, Fax (0 61 31) 7054 52, E-Mail Eberle.ce@zdf.de

118. Ebsen, Dr. Ingwer, Professor, Alfred-Mumbächer-Str. 19, 55128 Mainz, (0 61 31) 3310 20;

FB Rechtswissenschaft, Universität Frankfurt, Postfach 1119 32, 60054 Frankfurt am Main, (0 69) 798227 03, E-Mail Ebsen@jur.uni-frankfurt.de

119. Eckhoff, Dr. Rolf, Professor, Bornwiesweg 37, 65388 Schlangenbad-Georgenborn, (0 61 29) 4893 70, Fax (0 61 29) 4893 72;

Lehrstuhl für Öffentliches Recht, insbesondere Finanz- und Steuerrecht, Universitätsstr. 31, 93040 Regensburg, (09 41) 94326 56/57, Fax (09 41) 94319 74, E-Mail Rolf.Eckhoff@jura.uni-regensburg.de

120. Egli, Dr. Patricia, LL.M. (Yale), Privatdozentin, Lehrbeauftragte an der Universität St. Gallen, Meienbergstr. 65, CH-8645 Jona, (0041) 797689465 , E-Mail patricia.egli@unisg.ch 
121. Ehlers, Dr. Dirk, Professor, Am Mühlenbach 14, 48308 Senden, (0 25 97) 84 15, Institut für öffentliches Wirtschaftsrecht, Westfälische Wilhelms-Universität Münster, Universitätsstr. 14-16, 48143 Münster, (02 51) 83-2 27 01, Fax (02 51) 83-2 83 15, E-Mail ehlersd@uni-muenster.de

122. Ehmke, Dr. Horst, o. Professor, Am Römerlager 4, 53117 Bonn

123. Ehrenzeller, Dr. Bernhard, o. Professor, Kirchlistraße 36a, CH-9010 St. Gallen; Institut für Rechtswissenschaft und Rechtspraxis (IRP-HSG), Bodanstr. 4, CH-9000 St. Gallen, (00 41) 71-2 242440 oder -46, Fax (00 41) 71-2 2424 41, E-Mail Bernhard.Ehrenzeller@unisg.ch

124. Eifert, Dr. Martin, Professor, Beethovenstr. 57, 53115 Bonn; Justus-Liebig-Universität Gießen, Professur für Öffentliches Recht II, Hein-Heckroth-Straße 5, 35390 Gießen, (06 41) 99210 90, Fax (06 41) 99210 99, E-Mail martin.eifert@recht.uni-giessen.de

125. Ekardt, Dr. Felix, LL.M., M.A., Professor, Könneritzstraße 41, 04229 Leipzig;

Universität Rostock, apl. Professur für öffentliches Recht und Rechtsphilosophie, Möllner Str. 10, 18109 Rostock, (03 41) 92608 83, Fax (03 41) 92608 83, E-Mail felix.ekardt@uni-rostock.de

126. Elicker, Dr. Michael, Privatdozent, Dunzweiler Straße 6, 66564 Ottweiler, (0 68 58) 6998 53, Fax (0 68 58) 6998 53;

Universität des Saarlandes, Lehrstuhl für Staats- und Verwaltungsrecht, Wirtschafts-, Finanz- u. Steuerrecht, Im Stadtwald, 66123 Saarbrücken, (06 81) 3 02-21 04, Fax (06 81) 3 02-47 79, E-Mail m.elicker@gmx.de 
127. Emmerich-Fritsche, Dr. Angelika, Privatdozentin, Hornschuchpromenade 17, 90762 Fürth, (09 11) 7066 60;

E-Mailinfo@emmerich-fritsche.de

128. Enders, Dr. Christoph, Universitätsprofessor, Prellerstraße 1A, 04155 Leipzig, (03 41) 56433 71, Fax (03 41) 56433 72;

Universität Leipzig, Juristenfakultät, Lehrstuhl für Öffentliches Rechts, Staats- und Verfassungslehre, Burgstr. 21, 04109 Leipzig, (03 41) 9735 350, Fax (03 41) 9735 359, E-Mail chenders@rz-uni-leipzig.de

129. Engel, Dr. Christoph, Professor, Königsplatz 25, 53173 Bonn, (02 28) 95634 49, Fax (02 28) 95639 44; Max-Planck-Institut zur Erforschung von Gemeinschaftsgütern, Kurt-Schumacher-Straße 10, 53113 Bonn, (02 28) 914 16-10, Fax (02 28) 914 16-11, E-Mail engel@coll.mpg.de

130. Englisch, Dr. Joachim, Professor, Nettelbeckstr. 11, 40477 Düsseldorf, (02 11) 41658735 , E-Mail jo.e@gmx.de; Westfälische Wilhelms-Universität Münster, Lehrstuhl für Öffentliches Recht und Steuerrecht, Universitätsstr. 14-16, 48143 Münster, (02 51) 83-2 27 95, Fax (02 51) 83-2 83 86, E-Mail jengl_01@uni-muenster.de

131. Ennuschat, Dr. Jörg, Professor, Elberfelder Str. 23, 58452 Witten, (0 2302$) 3900$ 28;

Lehrstuhl für Öffentliches Recht und Europarecht, Universität Konstanz, Universitätsstraße 10, 78464 Konstanz, (0 75 31) 88-36 54, Fax (0 75 31) 88-21 94, E-Mail lehrstuhl.ennuschat@uni-konstanz.de 
132. Epiney, Dr. Astrid, Professorin, Avenue du Moléson 18, CH-1700 Fribourg, (00 41) 26-3 2342 24;

Universität Fribourg i.Ue./CH, Lehrstuhl für Europa-, Völker- und Öffentliches Recht, Av. de Beauregard 11, CH-1700 Fribourg, (00 41) 26-3 0080 90, Fax (00 41) 26-3 0097 76, E-Mail Astrid.Epiney@unifr.ch

133. Epping, Dr. Volker, Professor, Neddernwanne 38, 30989 Gehrden, (0 5108$) 9126$ 97;

Leibniz Universität Hannover, Juristische Fakultät, Königsworther Platz 1, 30167 Hannover, (05 11) 76282 48/49, Fax (05 11) 76282 52, E-Mail epping@jura.uni-hannover.de

134. Erbel, Dr. Günter, Professor, Burbacher Str. 10, 53129 Bonn;

Universität Bonn, Adenauerallee 24-42, 53113 Bonn, (02 28) 735583

135. Erbguth, Dr. Wilfried, Professor, Friedrich-Franz-Str. 38, 18119 Rostock-Warnemünde, (03 81) 54867 09, Fax (03 81) 54867 15; Universität Rostock, Juristische Fakultät, Richard-Wagner-Str. 31 (Haus 1), 18119 Rostock-Warnemünde, (03 81) 49882 11, Fax (03 81) 49882 12, E-Mail Wilfried.Erbguth@jurfak.uni-rostock.de

136. Erichsen, Dr. Hans-Uwe, o. Professor, Falkenhorst 17, 48155 Münster, (02 51) 313 12;

Kommunalwissenschaftliches Institut, Universität Münster, Universitätsstr. 14-16, 48143 Münster, (02 51) 8327 41, E-Mail erichse@uni-muenster.de

137. Errass, Dr. Christoph, Privatdozent, Rossfeldstr. 1, 3004 Bern, (0041) 3174121 17, E-Mail christoph.errass@bluewin.ch 
138. Faber, Dr. Angela, apl. Professorin, Am Dörnchesweg 42, 50259 Pulheim, (0 22 34) 643 70, Fax (0 22 34) 8029 93, E-Mail faber@fair-relations.de; Hauptreferentin beim Deutschen Städtetag, Lindenallee 13-17, 50968 Köln, (02 21) 37 71-1 72, Fax (02 21) 37 71-2 00, E-Mail angela.faber@staedtetag.de

139. Faber, Dr. Heiko, Professor, Wunstorfer Str. 1, 30989 Gehrden, (0 51 08) 22 34;

Universität Hannover, Königsworther Platz 1, 30167 Hannover, (05 11) 7 62-82 06, E-Mail faber@jura.uni-hannover.de

140. Faßbender, Dr. Bardo, LL.M. (Yale), Universitätsprofessor, Institut für Öffentliches Recht und Völkerrecht, Universität der Bundeswehr München, Werner-Heisenberg-Weg 39, 85577 Neubiberg, (0 89) 60 04-42 62, E-Mail fassbender@unibw.de

141. Faßbender, Dr. Kurt, Professor, Pölitzstr. 25, 04155 Leipzig, (03 41) 58201 18; Universität Leipzig, Lehrstuhl für Öffentliches Recht, insb. Umwelt- und Planungsrecht, Otto-Schill-Str. 2, 04109 Leipzig, (03 41 ) 97 35-1 31, Fax (03 41) 97 35-1 39, E-Mail fassbender@uni-leipzig.de

142. Fastenrath, Dr. Ulrich, Professor, Liliensteinstraße 4, 01277 Dresden, (03 51) 25405 36;

Juristische Fakultät der TU Dresden, Bergstr. 53, 01069 Dresden, (03 51) 46 33-73 33, Fax (03 51) 46 33-72 13, E-Mail quaas@jura.tu-dresden.de 
143. Fechner, Dr. Frank, Professor, Fischersand 57, 99084 Erfurt, (03 61) 64456 96;

TU Ilmenau, Institut für Rechtswissenschaft, Postfach 100 565, 98684 Ilmenau, (0 36 77) 69-40 22,

E-Mail Frank.Fechner@tu-ilmenau.de

144. Fehling, Dr. Michael, LL.M. (Berkeley), Professor, Farmsener Landstr. 39 B, 22359 Hamburg, (0 40) 609514 65, Bucerius Law School, Hochschule für Rechtswissenschaft, Jungiusstraße 6, 20355 Hamburg, Postfach 3010 30, (0 40) 307 06-2 31, Fax (0 40) 307 06-2 35, E-Mail michael.fehling@law-school.de

145. Feik, Dr. Rudolf, Ao. Univ.-Prof., Hans Sperl Straße 7, A-5020 Salzburg, (00 43) 67673043374 ;

Universität Salzburg, Fachbereich Öffentliches Recht, Kapitelgasse 5-7, A-5020 Salzburg, (00 43) 662804436 03, Fax (00 43) 662804436 29, E-Mail rudolf.feik@sbg.ac.at

146. Felix, Dr. Dagmar, Professorin, An den Fischteichen 47, 21227 Bendestorf, (0 41 83) 5006 67, Fax (0 41 83) 5007 29;

Universität Hamburg, Öffentliches Recht und Sozialrecht, Fakultät für Rechtswissenschaft, Rothenbaumchaussee 33, 20148 Hamburg, (0 40) 428 38-26 65, Fax (0 40) 428 38-29 30, E-Mail dagmar.felix@jura.uni-hamburg.de

147. Fetzer, Dr. Thomas, LL.M., Professor, Technische Universität Dresden, Juristische Fakultät, Lehrstuhl für Steuerrecht und Wirtschaftsrecht, 01062 Dresden, (0351) 46337 374, Fax (0351) 46337 798, E-Mail fetzer@jura.tu-dresden.de 
148. Fiedler, Dr. Wilfried, o. Professor, Am Löbel 2, 66125 Saarbrücken-Dudweiler, (0 68 97) 7664 01;

Forschungsstelle Internationaler Kulturgüterschutz, Universität des Saarlandes, Gebäude 16, Postfach 1511 50, 66041 Saarbrücken, (06 81) 3 02-32 00, Fax (06 81) 3 02-43 30, E-Mail w.fiedler@mx.uni-saarland.de

149. Fink, Dr. Udo, Univ.-Professor, Johannes-Gutenberg-Universität Mainz, Fachbereich Rechts- und Wirtschaftswissenschaften, 55099 Mainz, (0 61 31) 39223 84, E-Mail pfink@uni-mainz.de

150. Fisahn, Dr. Andreas, Professor, Grüner Weg 83, 32130 Enger;

Universität Bielefeld, Fakultät für Rechtswissenschaft, Postfach 1001 31, 33501 Bielefeld, (05 21) 1 06-43 84, E-Mail andreas.fisahn@uni-bielefeld.de

151. Fischer, Dr. Kristian, Privatdozent, Deidesheimer Str. 52, 68309 Mannheim, (06 21) 7382 45;

Lehrstuhl für Öffentliches Recht und Steuerrecht, Universität Mannheim, Schloss, Westflügel, 68131 Mannheim, (06 21) $181-1435$, Fax (06 21) 1 81-14 37, E-Mail kfischer@jura.uni-mannheim.de

152. Fischer-Lescano, Dr. Andreas, LL.M. (EUI, Florenz), Professor, Hobrechtsstr. 48, 12047 Berlin, Zentrum für Europäische Rechtspolitik (ZERP), Universität Bremen, Fachbereich Rechtswissenschaft, Universitätsallee GW 1, 28359 Bremen, (04 21) 21866222 , Fax (04 21) 21866 230, E-Mail fischer-lescano@zerp.uni-bremen.de 
153. Fleiner, Dr. Dr. h.c. Thomas, o. Professor, rte. Beaumont 9, $\mathrm{CH}-1700$ Fribourg, (00 41) 26-4 2466 94, Fax (00 41) 26-4 2466 89;

Institut für Föderalismus, Universität Fribourg, Route d' Englisberg 7, CH-1763 Granges-Paccot, (00 41) 26-3 008125 oder -28, Fax (00 41) 26-3 0097 24, E-Mail Thomas.Fleiner@unifr.ch

154. Folz, Dr. Hans-Ernst, Professor, Bispinger Weg 11, 30625 Hannover, (05 11) 575719 oder 5628 92;

Universität Hannover, Lehrstuhl für Öffentliches Recht, Völker- und Europarecht, Königsworther Platz 1, 30167 Hannover, (05 11) 7 62-82 48 oder -82 49, Fax (05 11) 7 62-82 52

155. Folz, Dr. Hans-Peter, Privatdozent, Christoph von Schmid-Straße 11, 86159 Augsburg, (08 21) 5894183 ;

Juristische Fakultät, Universität Augsburg, Universitätsstraße 24, 86159 Augsburg, (08 21) 59845 73, Fax (08 21) 59845 72, E-Mail Hans-Peter.Folz@jura.uni-augsburg.de

156. Frank, Dr. Dr. h.c. Götz, Professor, Cäcilienplatz 4, 26122 Oldenburg, (04 41) 756 89;

Carl von Ossietzky Universität Oldenburg, Juristisches Seminar, Öffentliches Wirtschaftsrecht, 26111 Oldenburg, Paketanschrift: Ammerländer Heerstraße 114-118, 26129 Oldenburg; (04 41) 7 98-41 43, Fax (04 41) 7 98-41 51, E-Mail Goetz.Frank@uni-oldenburg.de

157. Frankenberg, Dr. Dr. Günter, Professor, Buchrainweg 17, 63069 Offenbach; Institut für Öffentliches Recht, Goethe-Universität Frankfurt, Rechtswissenschaft, Grüneburgplatz 1, 60629 Frankfurt am Main, (0 69) 7983 4-2 70 oder -2 69, E-Mail Frankenberg@jur.uni-frankfurt.de 
158. Franzius, Dr. Claudio, Privatdozent, Goernestr. 13, 20249 Hamburg, (040) 238029 52;

Universität Hamburg, Fakultät für Rechtswissenschaft, Seminar für Öffentliches Rechts und Staatslehre, Rothenbaumchaussee 33, 20148 Hamburg, (040) 42838 5443, Fax (040) 428382930 , E-Mail claudio.franzius@jura.uni-hamburg.de

159. Friauf, Dr. Karl Heinrich, o. Professor, Eichenhainallee 17, 51427 Bergisch-Gladbach, (0 22 04) 619 84;

Universität Köln, 50923 Köln

160. Fromont, Dr. Dr. h.c. mult. Michel, Professor, 12, Boulevard de Port Royal, F-75005 Paris, (00 33) 145357371 , E-Mail Fromont.michel@wanadoo.fr

161. Frotscher, Dr. Werner, Professor, Habichtstalgasse 32, 35037 Marburg/Lahn, (0 64 21) 329 61; Universität Marburg, Universitätsstr. 6, 35032 Marburg/Lahn, (0 64 21) 28-2 31 22/1 26 (Sekr.), Fax (0 64 21) 2 82-38 40, E-Mail Frotsche@staff.uni-marburg.de

162. Frowein, Dr. Dr. h.c. Jochen Abr., o. Professor, Blumenthalstr. 53, 69120 Heidelberg, (0 62 21) 4746 82, Fax (0 62 21) 4139 71;

MPI für ausländisches öffentliches Recht und Völkerrecht, Im Neuenheimer Feld 535, 69120 Heidelberg, (0 62 21) 4 82-2 58, Fax (0 62 21) 4 82-6 77, E-Mail jfrowein@mpil.de

163. Frye, Dr. Bernhard, Richter am Finanzgericht, Privatdozent, (0361) 34621 04;

Thüringer Finanzgericht, Bahnhofstr. 3 a, 99867 Gotha, (03621) 432 -221, -235, Fax (03621) 432 -199, E-Mail b.frye@gmx.de 
164. Führ, Dr. Martin, Professor, Rostockerstr. 17, 63303 Dreieich/Sprendlingen, (0 6103 ) 9366 17, Fax (0 61 03) 9366 19;

Fachhochschule Darmstadt, Haardtring 100, 64295 Darmstadt, E-Mail fuehr@sofia-darmstadt.de

165. Funk, Dr. Bernd-Christian, o. Professor, Franz Grassler Gasse 23, A-1230 Wien, (00 43) 188929 35, Fax (00 43) 188929 35;

Institut für Staats- und Verwaltungsrecht, Universität Wien, Juridicum, Schottenbastei 10-16, A-1010 Wien;

E-Mail bernd-christian.funk@univie.ac.at Institut für Universitätsrecht und Universitätsmanagement, Johannes Kepler Universität Linz, Altenbergerstr. 69, A-4040 Linz, (00 43) 7 32-24 68-93 36, Fax (00 43) 7 32-24 6893 99, E-Mail bernd-christian.funk@jku.at

166. Funke, Dr. Andreas, Privatdozent, Lechenicher Str. 18, 50937 Köln, (0221) 374829;

Universität zu Köln, Institut für Völkerrecht und ausländisches öffentliches Recht, Gottfried-Keller-Str. 2, 50931 Köln, (0221) 470 -2616, Fax (0221) 470 -4992, E-Mail andreas.funke@uni-koeln.de

167. Gächter, Dr. Thomas, Professor, Ausserdorferstr. 12g, CH-8052 Zürich, (0041) 136337 24;

Universität Zürich, Treichlerstr. 10, CH-8032 Zürich, (00 41) 4463430 62,

E-Mail thomas.gaechter@rwi.unizh.ch

168. Gaitanides, Dr. Charlotte, LL.M. (Barcelona), Professorin, 22041 Hamburg, (0 40) 682848 77;

Universität Flensburg, Internationales Institut für Management, Campusallee 3, 24943 Flensburg, (0461) 805 -2766, Fax (0461) 805 -2561

E-Mail charlotte.gaitanides@uni-flensburg.de 
169. Galetta, Dr. Diana-Urania, LL.M., Professorin, Via Galla Placidia 6, I-20131 Milano;

Università degli Studi di Milano, Dipartimento giuridico-politico, Via Conservatorio 7, I-20122 Milano, (0039) 02-503 212 59, Fax (0039) 02-503 212 80, E-Mail diana.galetta@unimi.it

170. Gallwas, Dr. Hans-Ullrich, Universitätsprofessor, Hans-Leipelt-Str. 16, 80805 München, (0170) 21672 08;

Obermaisperg, 84323 Massing, (08724) 1386, E-Mail hu-gallwas@t-online.de; Universität München, Professor-Huber-Platz 2, 80539 München

171. Gamper, Dr. Anna, Univ.-Prof., Universität Innsbruck, Institut für Öffentliches Recht, Staats- und Verwaltungslehre, Innrain 52d, A-6020 Innsbruck, (00 43) 5125 07-82 24, Fax (00 43) 5125 07-28 28, E-Mail Anna.Gamper@uibk.ac.at

172. Gärditz, Dr. Klaus Ferdinand, Professor, Kastanienweg 48, 53177 Bonn, (02 28) 280527 97;

Rheinische Friedrich-Wilhelms-Universität Bonn, Institut für Öffentliches Recht, Adenauerallee 24-42, 53113 Bonn, (02 28) 73-91 76, E-Mail gaerditz@jura.uni-bonn.de

173. Gas, Dr. Tonio, Professor, Bertolt-Brecht-Str. 59, 49088 Osnabrück, E-Mail tgas@uos.de; Niedersächsisches Studieninstitut für kommunale Verwaltung e.V., Kommunale Hochschule für Verwaltung in Niedersachsen, Professur für Staats-, Verfassungs- und Europarecht, Wielandstr. 8, 30169 Hannover, (0511) 1609-448, Fax (0511) 15 537, E-Mail tonio.gas@nds-sti.de 
174. Gassner, Dr. Ulrich M., Mag.rer.publ., M.Jur. (Oxon), Professor, Scharnitzer Weg 9, 86163 Augsburg, (08 21) 632 50, E-Mail ugassner@web.de;

Universität Augsburg, Universitätsstr. 2, 86135 Augsburg, (08 21) 5 98-45 46, Fax (08 21) 5 98-45 47, E-Mail Ulrich.Gassner@jura.uni-augsburg.de

175. Geis, Dr. Max-Emanuel, o. Professor, Valentin-Rathgeber-Str. 1, 96049 Bamberg, (09 51) 51 93-3 05 oder -3 06, Fax (09 51) 51 93-3 08;

Friedrich-Alexander-Universität Erlangen, Institut für Staats- und Verwaltungsrecht, Schillerstr. 1, 91054 Erlangen, (0 91 31) 85228 18, Fax (0 91 31) 85263 82, E-Mail max-emanuel.geis@jura.uni-erlangen.de

176. Gellermann, Dr. Martin, apl. Professor, Schlesierstraße 14, 49492 Westerkappeln, (0 54 04) 20 47, Fax (0 54 04) 9194 75; Universität Osnabrück, Fachbereich Rechtswissenschaften, 49069 Osnabrück, (0 54 04) 9196 95, E-Mail M.Gellermann@t-online.de

177. Germann, Dr. Michael, Professor, Rathenauplatz 13, 06114 Halle, (03 45) 52389 32;

Martin-Luther-Universität Halle-Wittenberg, Lehrstuhl für Öffentliches Recht, Staatskirchenrecht und Kirchenrecht, Universitätsplatz 5, 06108 Halle, (03 45) 55-2 32 20, Fax (03 45) 55-2 76 74, E-Mail Germann@jura.uni-halle.de

178. Gersdorf, Dr. Hubertus, Professor, Alte Leipziger Str. 10, 10117 Berlin, (0 30) 206196 61, Fax (0 30) 206196 62; Universität Rostock, Juristische Fakultät, Gerd Bucerius-Stiftungsprofessur, Richard-Wagner-Straße 7, 18055 Rostock, (03 81) 20360 76, Fax (03 81) 20360 75, E-Mail hubertus.gersdorf@uni-rostock.de 
179. Giegerich, Dr. Thomas, Professor, LL.M. (University of Virginia), Birkenweg 90, 24211 Preetz;

Walther-Schücking-Institut für Internationales Recht, Universität Kiel,

Westring 400, 24098 Kiel, (04 31) 8 80-21 89 (-17 33 [Sekr.]),

E-Mail tgiegerich@internat-recht.uni-kiel.de

180. Goerlich, Dr. Helmut, Professor,

Universität Leipzig,

Institut für Staats- und Verwaltungsrecht,

Burgstr. 27, 04109 Leipzig,

(03 41) 97-3 51 71, Fax (03 41) 97-3 51 79,

E-Mail helmut.goerlich@gmx.de

181. Gornig, Dr. Dr. h c. mult. Gilbert, Professor,

Pfarracker 4, 35043 Marburg-Bauerbach,

(0 64 21) 1635 66, Fax (0 64 21) 1637 66;

Institut für Öffentliches Recht, Universität Marburg,

Universitätsstr. 6, 35032 Marburg,

(0 64 21) 2823131 oder 28-31 27, Fax (0 64 21) 28238 53,

E-Mail Gornig@voelkerrecht.com

182. Görisch, Dr. Christoph, Privatdozent,

Martin-Niemöller-Str. 61,

48159 Münster;

Westfälische Wilhelms-Universität, Institut für Öffentliches Recht und Politik,

Wilmergasse 28, 48143 Münster,

(02 51) 83-2 18 61, Fax (02 51) 510 49-19

E-Mail gorisch@uni-muenster.de

183. Götz, Dr. Volkmar, o. Professor,

Geismarlandstr. 17a, 37083 Göttingen,

(05 51) 431 19;

Universität Göttingen,

Abt. Europarecht des Instituts für Völkerrecht,

Platz der Göttinger Sieben 5, 37073 Göttingen,

(05 51) 39-47 61, Fax (05 51) 39-21 96,

E-Mail europa@uni-goettingen.de 
184. Grabenwarter, Dr. Dr. Christoph, Universitätsprofessor, Institut für Österreichisches und Europäisches Öffentliches Recht, Wirtschaftsuniversität Wien, Althanstr. 39-45, A-1090 Wien, (00 43) 1313 36-44 23, Fax (00 43) 1313 36-92 05;

Mitglied des Verfassungsgerichtshofs, Verfassungsgerichtshof, Judenplatz 11, A-1010 Wien, (00 43) 1531 22,

E-Mail christoph.grabenwarter@wu-wien.ac.at

185. Gramlich, Dr. Ludwig, Professor, Justus-Liebig-Str. 38 A, 64839 Münster;

Fakultät für Wirtschaftswissenschaften, TU Chemnitz-Zwickau, Postfach 9 64, 09009 Chemnitz, (03 71) 53141 64, -65, Fax (03 71) 53139 61, E-Mail l.gramlich@wirtschaft.tu-chemnitz.de

186. Gramm, Dr. Christof, Privatdozent, MinRat, Wilhelmstraße 10, 53604 Bad Honnef, (0 22 24) 48 34;

Bundesministerium der Verteidigung, Postfach 1328, 53003 Bonn, (02 28) 12-93 70, E-Mail ChristofGramm@bmvg.bund.de

187. Graser, Dr. Alexander, Professor, Brennereistraße 66, 85662 Hohenbrunn, (0 8102$) 7788$ 55;

Hertie School of Governance, Schlossplatz 1, 10178 Berlin, (0 30) 2123123 14, Fax (0 30) 2123128 88, E-Mail graser@hertie-school.org

188. Grawert, Dr. Dr. h.c. Rolf, o. Professor, Aloysiusstrasse 28, 44795 Bochum, (02 34) 4736 92, Fax (02 34) 51691 36;

Ruhr-Universität Bochum, Juristische Fakultät, Universitätsstrasse 150, GC 8/59, 44721 Bochum, (02 34) 322252 65, Fax (02 34) 32142 36, E-Mail Rolf.Grawert@ruhr-uni-bochum.de 
189. Grewe, Dr. Constance, Universitätsprofessorin, 4 Rue Louis Apffel - F 67000 Strasbourg, (00 33) 388373784 , E-Mail Constance.Grewe@orange.fr Université de Strasbourg, Faculté de droit 1 Place d'Athénes, BP 66, F-67045 Strasbourg Cedex

190. Grewlich, Dr. Dr. Klaus W., LL.M. (Berkeley), Professor, Botschafter der Bundesrepublik Deutschland a.D., Colmantstr. 43, 53115 Bonn;

Rechts- und Staatswissenschaftliche Fakultät und Zentrum für Europäische Integrationsforschung (ZEI), Rheinische Friedrich-Wilhelms-Universität, Bonn; Hertie School of Governance, Berlin; Europakolleg Brügge \& Warschau/Schloss Natolin; E-Mailkwgr@gmx.de

191. Grigoleit, Dr. Klaus Joachim, Universitätsprofessor, Eisenacher Str. 65, 10823 Berlin; TU Dortmund, Fakultät Raumplanung, Fachgebiet Raumplanungs- und Umweltrecht, August-Schmidt-Straße 10, 44227 Dortmund, (0231) 75532 17, Fax (0231) 75534 24, E-Mail grigoleit@kjgrigoleit.de

192. Griller, Dr. Stefan, Universitätsprofessor, Hungerbergstr. 11-13, A-1190 Wien, (00 43) 13224 05;

Europainstitut, Wirtschaftsuniversität Wien, Althanstr. 39-45, A-1090 Wien, (00 43) 1313 36-41 35 oder 41 36, Fax (00 43) 1313 36-7 58, E-Mail Stefan.Griller@wu-wien.ac.at

193. Grimm, Dr. Dr. h.c. mult., LL.M. (Harvard),

Dieter, o. Professor (em.), Humboldt-Universität zu Berlin, Juristische Fakultät, Unter den Linden 6, 10099 Berlin, (0 30) 20 93-35 66, Fax (0 30) 20 93-35 78;

Wissenschaftskolleg zu Berlin, Institute for Advanced Study, Wallotstr. 19, 14193 Berlin,

(0 30) 890 01-0 (Zentrale), (0 30) 890 01-1 24, Fax (0 30) 890 01-1 00, E-Mail grimm@wiko-berlin.de 
194. Gröpl, Dr. Christoph, Professor, Lehrstuhl für Staats- und Verwaltungsrecht, Rechts- und Wirtschaftswissenschaftliche Fakultät, Universität des Saarlandes, Postfach 1511 50, 66041 Saarbrücken, (06 81) 3 02-32 00, Fax (06 81) 3 02-43 30, E-Mail groepl@mx.uni-saarland.de

195. Gröschner, Dr. Rolf, o. Professor, Stormstr. 39, 90491 Nürnberg, (09 11) 5914 08;

Rechtswissenschaftliche Fakultät, Universität Jena, Carl-Zeiss-Straße 3, 07743 Jena, (0 36 41) 942220 oder -21, Fax (0 36 41) 9422 22, E-Mail r.groeschner@recht.uni-jena.de

196. Groh, Dr. Kathrin, Privatdozentin, Rohrteichstr. 44, 33602 Bielefeld, (05 21) 56004 45;

Universität Bielefeld, Fakultät für Rechtswissenschaft, Universitätsstr. 25, 33615 Bielefeld, (05 21) 1 06-43 97, Fax (05 21) 1 06-15 43 97, E-Mail kathgroh@gmx.de

197. Gromitsaris, Dr. Athanasios, Privatdozent, E-Mail gromitsaris@hotmail.com;

Friedrich-Schiller-Universität, Rechtswissenschaftliche Fakultät, Carl-Zeiss-Str. 3, 07737 Jena, (0 36 41) 942230 , E-Mail R.Ullrich@recht.uni-jena.de

198. Groß, Dr. Thomas, Professor, Universität Osnabrück, European Legal Studies Institute, Süsterstr. 28, 49069 Osnabrück, (0541) 969 - 4500, E-Mail thgross@uni-osnabrueck.de 
199. Grote, Dr. Rainer, LL.M. (Edinburgh), Privatdozent, Im Sand 3A, 69115 Heidelberg, (0 62 21) 1643 46, Fax (0 62 21) 9147 35;

Max-Planck-Institut für ausländisches öffentliches Recht und Völkerrecht, Im Neuenheimer Feld 535, 69120 Heidelberg, (0 62 21) 4822 44, Fax (0 62 21) 4822 88, E-Mail rgrote@mpil.de

200. Grupp, Dr. Klaus, Universitätsprofessor, Stephanieufer 5, 68163 Mannheim, (06 21) 8221 97, Fax (06 21) 8221 97;

Universität des Saarlandes, Rechts- und Wirtschaftswissenschaftliche Fakultät, Abteilung Rechtswissenschaft, Postfach 1511 50, 66041 Saarbrücken, (06 81) 3 02-48 53, Fax (06 81) 3 02-39 49, E-Mail k.grupp@mx.uni-saarland.de

201. Grzeszick, Dr. Bernd, LL.M. (Cambridge), Professor, Henkestraße 74-76, 91052 Erlangen, (0 91 31) 12328 14, E-Mail Grzeszick@web.de; Universität Heidelberg, Institut für Öffentliches Recht, Verfassungslehre und Rechtsphilosophie, Friedrich-Ebert-Anlage 6-10, 69117 Heidelberg, (06221) 547432

202. Guckelberger, Dr. Annette, Professorin, Lehrstuhl für Öffentliches Recht, Rechts- und Wirtschaftswissenschaftliche Fakultät, Universität des Saarlandes, Postfach 1511 50, 66041 Saarbrücken, (06 81) 3 02-5 74 01, E-Mail a.guckelberger@mx.uni-saarland.de

203. Gundel, Dr. Jörg, Professor,

Lehrstuhl für Öffentliches Recht, Völker- und Europarecht, Universität Bayreuth, 95440 Bayreuth, (09 21) 55-29 43, E-Mail joerg.gundel@uni-bayreuth.de 
204. Gurlit, Dr. Elke, Universitätsprofessorin, Rüdesheimer Straße 18, 65197 Wiesbaden, (06 11) 375152 oder (01 79) 59222 15;

Fachbereich Rechts- und Wirtschaftswissenschaft, Johannes Gutenberg-Universität Mainz, Jakob-Welder-Weg 9, 55099 Mainz, (0 61 31) 39231 14, Fax (0 61 31) 39240 59, E-Mail gurlit@uni-mainz.de

205. Gusy, Dr. Christoph, Professor, Wendischhof 14, 33619 Bielefeld, (05 21) 96779 67;

Universität Bielefeld, Fakultät für Rechtswissenschaft, Universitätsstr. 25, 33615 Bielefeld, (05 21) 10643 97, Fax (05 21) 10680 61, E-Mail christoph.gusy@uni-bielefeld.de

206. Haack, Dr. Stefan, Professor, Rheinische Friedrich-Wilhelms-Universität Bonn, Professur für Öffentliches Recht, Adenauerallee 18-22, 53113 Bonn, (02 28) 73-62 411, E-Mail haack@jura.uni-bonn.de E-Mail StefanHaack@web.de

207. Häberle, Dr. Dr. h.c. mult. Peter, o. Professor, Forschungsstelle für Europäisches Verfassungsrecht, Universität Bayreuth, Universitätsstraße 30, Postfach, 95440 Bayreuth, (09 21) 5570 88, Fax (09 21) 5570 99, E-Mail Peter.Haeberle@uni-bayreuth.de

208. Häde, Dr. Ulrich, Universitätsprofessor, Lennéstraße 15, 15234 Frankfurt (Oder), (03 35) 6857438 ;

Europa-Universität Viadrina, Lehrstuhl für Öffentliches Recht, insb. Verwaltungsrecht, Finanzrecht und Währungsrecht, Postfach 17 86, 15207 Frankfurt/Oder, Hausanschrift: Große Scharrnstr. 59, 15230 Frankfurt (Oder), (03 35) 55 34-26 70, Fax (03 35) 55 34-25 25,

E-Mail haede@euv-frankfurt-o.de 
209. Haedrich, Dr. Martina, Professorin, Im Ritzetal 20, 07749 Jena, (0 36 41) 4485 25, Fax (0 36 41) 4444 14;

Rechtswissenschaftliche Fakultät, Friedrich-Schiller-Universität, Carl-Zeiss-Straße 3, 07743 Jena, (0 36 41) 9422 15, Fax (0 36 41) 942 002, E-Mail m.haedrich@recht.uni-jena.de

210. Hafner, Dr. Felix, Professor, Hirzbrunnenschanze 67, CH-4058 Basel, (00 41) 61-6 9140 64;

Universität Basel, Lehrstuhl für Öffentliches Recht, Peter Merian-Weg 8, Postfach, 4002 Basel, (00 41) 6126725 64, Fax (00 41) 6126707 95, E-Mail Felix.Hafner@unibas.ch

211. Hailbronner, Dr. Kay, o. Professor, Toggenbühl, CH-8269 Fruthwilen, (00 41) 71-6 6419 46, Fax (00 41) 71-6 6416 26;

Universität Konstanz, (0 75 31) 8822 47, E-Mail migration@uni-konstanz.de

212. Hain, Dr. Karl-E., Professor, Herrenstr. 10, 57627 Hachenburg, (0 26 62) 9420 64; Universität zu Köln, Institut für Medienrecht und Kommunikationsrecht, Lehrstuhl für Öffentliches Recht und Medienrecht, Aachener Str. 197-199, 50931 Köln, (02 21) 285 56-1 12, Fax (02 21) 2 85 56-1 22, E-Mail haink@uni-koeln.de

213. Haller, Dr. Herbert, Universitätsprofessor, Felix-Mottl-Str. 48, Haus 2, A-1190 Wien, (00 43) 134293 82;

Wirtschaftsuniversität Wien, (00 43) 13133646 68, E-Mail h.haller@vfgh.gv.at 
214. Haller, Dr. Walter, o. Professor, Burgstrasse 264, $\mathrm{CH}-8706$ Meilen, (00 41) 4492310 14;

Forchstr. 59, CH-8032 Zürich, (00 41) 433439600 , E-Mail w-haller@bluewin.ch

215. Haltern, Dr. Ulrich, LL.M. (Yale), Universitätsprofessor, Bölschestr. 2, 30173 Hannover, (05 11) 3576259 ;

Universität Hannover, Lehrstuhl für deutsches und europäisches Staats- und Verwaltungsrecht, Königsworther Platz 1, 30167 Hannover, (05 11) 76281 86, Fax (05 11) 7628173 , E-Mail LS.Haltern@jura.uni-hannover.de

216. Hammer, Dr. Felix, apl. Prof., Gelber Kreidebusen 33/5, 72108 Rottenburg;

Justitiar und Kanzler der Diözese Rottenburg-Stuttgart, Bischöfliches Ordinariat, Eugen-Bolz-Platz 1, 72108 Rottenburg, (0 74 72) 169361 Fax (0 74 72) 169833 61, E-Mail kanzler@bo.drs.de

217. Hammer, Dr. Stefan, Univ.-Doz., Anton Frank-Gasse 17, A-1180 Wien, (00 43) 147059 76;

Universität Wien, Institut für Staats- und Verwaltungsrecht, Schottenbastei 10-16, A-1010 Wien, (00 43) 142 77-3 54 65, Fax (00 43) 142 77-3 54 69, E-Mail stefan.hammer@univie.ac.at

218. Hangartner, Dr. Yvo, o. Professor, Am Gozenberg 2, CH-9202 Gossau, (00 41) 71-85 15 11; Hochschule St. Gallen

219. Hänni, Dr. Peter, o. Professor, Stadtgraben 6, CH-3280 Murten, (00 41) 26-6 7058 15;

Lehrstuhl für Staats- und Verwaltungsrecht, Universität Fribourg, Les Portes de Fribourg, Route d'Englisberg 7, $\mathrm{CH}-1763$ Granges-Paccot, (00 41) 26-3 0081 29, Fax (00 41) 26-3 0097 24, E-Mail Peter.Haenni@unifr.ch 
220. Hanschel, Dr. Dirk, M.C.L., Privatdozent, Hauptstr. 154, 69117 Heidelberg, (06221) 166 958, hanschel@googlemail.com; Universität Mannheim, Fakultät für Rechtswissenschaft und Volkswirtschaftslehre, 68131 Mannheim

221. Haratsch, Dr. Andreas, Universitätsprofessor, Berliner Straße 18c, 58313 Herdecke, (0 23 30) 9267 13; Lehrstuhl für Deutsches und Europäisches Verfassungs- und Verwaltungsrecht sowie Völkerrecht, FernUniversität in Hagen, Universitätsstraße 21, 58084 Hagen, (0 23 31) 9 87-28 77 oder -43 89, Fax (0 23 31) 9 87-3 24, E-Mail Andreas.Haratsch@fernuni-hagen.de

222. Härtel, Dr. Ines, Professorin, Schinkelstr. 13, 44801 Bochum, (02 34) 89033 63;

Ruhr-Universität Bochum, Lehrstuhl für Öffentliches Recht, Verwaltungs-, Europa-, Agrar- und Umweltrecht, GC 8/39, Universitätsstr. 150, 44780 Bochum, (02 34) 32222 65, Mobil (01 79) 66364 22, E-Mail LS-Haertel@ruhr-uni-bochum.de

223. Hartmann, Dr. Bernd J., LL.M.(Virginia), Privatdozent, Max-Planck-Str. 5 B, 45657 Recklinghausen, (02361) 9377 259, Fax (02361) 4075 186;

Universität Münster, Institut für Öffentliches Recht und Politik, Wilmergasse 28, 48143 Münster, (0251) 51049 0, Fax (0251) 51049 19,

E-Mail hartmab@uni-muenster.de

224. Hase, Dr. Friedhelm, Professor, Bandelstraße 10 b, 28359 Bremen, (0421) 242784 40;

Universität Bremen,

Fachbereich 6, Rechtswissenschaft, Universitätsallee, 28359 Bremen, (0421) 218-66 010, Fax (0421) 218-66 052, E-Mail fhase@uni-bremen.de 
225. Hatje, Dr. Armin, Professor, Universität Hamburg, Fakultät für Rechtswissenschaft, Abt. Europäisches Gemeinschaftsrecht, Schlüterstr. 28, 20146 Hamburg, (0 40) 428 38-30 46, Fax (0 40) 428 38-43 67, E-Mail armin.hatje@jura.uni-hamburg.de

226. Hebeler, Dr. Timo, Professor, Universität Trier, Professur für Öffentliches Recht, 54286 Trier, (06 51) 2012 588, E-Mail hebeler@uni-trier.de

227. Heckel, Dr. iur. Dr. theol. h.c. Martin, o. Universitätsprofessor, Lieschingstr. 3, 72076 Tübingen, (0 70 71) 61427

228. Hecker, Dr. Jan, LL.M. (Cambridge), apl. Professor, Richter am Bundesverwaltungsgericht, Platanenstr. 25, 13156 Berlin, (0176) 232928 26;

Bundesverwaltungsgericht, Simsonplatz 1, 04107 Leipzig, (0341) 2007-2065,

E-Mail jan.hecker@bverwg.bund.de

229. Heckmann, Dr. Dirk, Universitätsprofessor, stv. Mitglied des Bayerischen Verfassungsgerichtshofs, Schärdinger Straße 11e, 94032 Passau, (08 51) 7538 83, Fax (08 51) 49058 20;

Universität Passau, Ordinarius für Internet- und Sicherheitsrecht, Innstraße 40, 94032 Passau, (08 51) 5 09-22 90, Fax (08 51) 5 09-22 92, E-Mail Heckmann@uni-passau.de

230. Heinig, Dr. Hans Michael, Professor, Rochstr. 17, 10178 Berlin, (01 71) 64611 21; Kirchenrechtliches Institut der EKD, Goßlerstr. 11, 37073 Göttingen, (05 51) 39-1 06 02; Universität Göttingen, Lehrstuhl für Öffentliches Recht, insb. Kirchen- und Staatskirchenrecht, Goßlerstr. 11, 37073 Göttingen, (05 51) 39-1 06 02, Fax (05 51) 39-1 06 07, E-Mail info@kirchenrechtliches-institut.de 
231. Heintschel von Heinegg, Dr. Wolff, Professor, Europa-Universität Viadrina, Frankfurt (Oder), Lehrstuhl für Öffentliches Recht, August-Bebel-Str. 12, 15234 Frankfurt (Oder), (03 35) 55 34-29 16, Fax (03 35) 55 34-29 15, E-Mail Heinegg@euv-frankfurt-o.de

232. Heintzen, Dr. Markus, Professor, Freie Universität Berlin, Fachbereich Rechtswissenschaft, Van't-Hoff-Str. 8, 14195 Berlin, (0 30) 8 38-5 24 79, Fax (0 30) 8 38-5 21 05, E-Mail Heintzen@zedat.fu-berlin.de

233. Heitsch, Dr. Christian, apl. Professor, 72 Queens Road, Caversham, Reading, Berks., RG4 8DL, U.K., (00 44) 11894749 13, E-Mail c_heitsch@web.de; Lecturer in Law, Brunel Law School, Brunel University West London, Kingston Lane, Uxbridge, Middlesex UB8 3PH, United Kingdom, (00 44) 18952676 50,

E-Mail christian.heitsch@brunel.ac.uk

234. Hellermann, Dr. Johannes, Universitätsprofessor, Hardenbergstr. 12a, 33615 Bielefeld, (05 21) 1600 38;

Universität Bielefeld, Fakultät für Rechtswissenschaft, Universitätsstr. 25, 33615 Bielefeld, (05 21) 1 06-44 22, Fax (05 21) 1 06-60 48, E-Mail Johannes.Hellermann@uni-bielefeld.de

235. Hendler, Dr. Reinhard, Universitätsprofessor, Laurentius-Zeller-Str. 12, 54294 Trier, (06 51) 93729 44;

Universität Trier, Fachbereich Rechtswissenschaft, Universitätsring 15, 54286 Trier, (06 51) 2 01-25 56 oder 25 58, Fax (06 51) 2 01-39 03, E-Mail Hendler@uni-trier.de 
236. Hengstschläger, Dr. Johannes, o. Universitätsprofessor, Steinfeldgasse 7, A-1190 Wien, (00 43) $132-817$ 27;

Johannes-Kepler-Universität, Altenbergerstr. 69, A-4040 Linz, (00 43) 7 32-24 68-4 01, Fax (00 43) 7 32-2 46 43,

E-Mail johannes.hengstschlaeger@jku.at

237. Hense, Dr. Ansgar, Privatdozent, Austraße 5, 53179 Bonn, (02 28) 42953 72;

Institut für Staatskirchenrecht der Diözesen Deutschlands, Adenauerallee 19, 53111 Bonn, (02 28) 26 74-3 61, E-Mail ansgar.hense@web.de

238. Herbst, Dr. Tobias, Privatdozent, Seehofstr. 116, 14167 Berlin, (030) 8171104 ;

Humboldt-Universität zu Berlin, Juristische Fakultät, Unter den Linden 6, 10099 Berlin, (030) 2093 -3426 oder -3381, Fax (030) 2093 -3345,

E-Mail tobias.herbst@rewi.hu-berlin.de

239. Herdegen, Dr. Matthias, Professor, Friedrich-Wilhelm-Str. 35, 53113 Bonn;

Rechts- und Staatswissenschaftliche Fakultät, Universität Bonn, Adenauerallee 44, 53113 Bonn, (02 28) 7355 70/-80, Fax (02 28) 7379 01, E-Mail Herdegen@uni-bonn.de

240. Hermes, Dr. Georg, Professor, Berliner Str. 14a, 61440 Oberursel, (0 61 71) 50819 91, Fax (0 61 71) 69475 70;

Universität Frankfurt, Fachbereich Rechtswissenschaft, Campus Westend, Grüneburgplatz 1 (RuW), Postfach 1119 31, 60054 Frankfurt am Main, (0 69) 7 98-3 42 75, Fax (0 69) 7 98-3 45 12, E-Mail G.Hermes@jur.uni-frankfurt.de 
241. Herrmann, Dr. Christoph, LL.M., Professor, Florianstr. 18, 94034 Passau, (0851) 23020 18, (01 77) 84275 43;

Universität Passau, Lehrstuhl für Staats- und Verwaltungsrecht, Europarecht, Europäisches und Internationales Wirtschaftsrecht, Innstraße 39, 94032 Passau, (0851) 509-2330, Fax (0851) 509-2332, E-Mail christoph.herrmann@eui.eu

242. Herrmann, Dr. Günter, Professor, Intendant i.R. Wankweg 13, 87642 Buching/Allgäu, (0 83 68) 16 96; Fax (08368) 1297 herrmann.medienrecht@t-online.de

243. Herzog, Dr. Roman, Professor, Bundespräsident a.D., Im Stahlbühl 20,74074 Heilbronn, (07131) 3952 160, Fax (07131) 3952 168, E-Mail buerobprherzog@gmx.de

244. Heselhaus, Dr. Sebastian, Professor, M.A., Kehlhofweg 10, CH-6043 Adligenswil, Schweiz, (00 41) 4137025 00;

Universität Luzern, Rechtswissenschaftliche Fakultät, Lehrstuhl für Europarecht, Völkerrecht und Öffentliches Recht, Hofstr. 9, Postfach 7464, CH-6000 Luzern 7, Schweiz, (00 41) 4122874 11, Fax (00 41) 412287431

E-Mail sebastian.heselhaus@unilu.ch

245. Heun, Dr. Werner, Professor,

Bürgerstraße 5, 37073 Göttingen, (05 51) 7062 48;

Universität Göttingen, Institut für Allgemeine Staatslehre und Politische Wissenschaften, Goßlerstraße 11, 37073 Göttingen, (05 51) 39-46 93, Fax (05 51) 39-22 39, E-Mail staats1@gwdg.de

246. Hey, Dr. Johanna, Professorin, Wiethasestraße 73, 50933 Köln, (02 21) 49117 38, Fax (02 21) 49117 34;

Universität zu Köln, Institut für Steuerrecht, Albertus-Magnus-Platz, 50923 Köln, (02 21) 4 70-22 71, Fax (02 21) 4 70-50 27, E-Mail johanna.hey@uni-koeln.de 
247. Heyen, Dr. iur. Lic. phil. Erk Volkmar, Universitätsprofessor, Arndtstraße 22, 17489 Greifswald, (0 38 34) 5027 16;

Ernst Moritz Arndt-Universität, Domstr. 20, 17489 Greifswald, E-Mail lsheyen@uni-greifswald.de

248. Hidien, Dr. Jürgen W., Professor, Goebenstr. 33, 48151 Münster, (02 51) 47877

249. Hilf, Dr. Meinhard, Universitätsprofessor, Bahnsenallee 71, 21465 Reinbek bei Hamburg, (0 40) 781075 10, Fax (0 40) 781075 12; Bucerius Law School, Jungiusstraße 6, 20355 Hamburg, (0 40) 307 06-1 58, Fax (0 40) 307 06-2 46, E-Mail meinhard.hilf@law-school.de

250. Hill, Dr. Hermann, Professor, Kilianstraße 5, 67373 Dudenhofen;

Deutsche Hochschule für Verwaltungswissenschaften Speyer, Postfach 14 09, 67324 Speyer, (0 62 32) 6 54-3 28, E-Mail Hill@dhv-speyer.de

251. Hillgruber, Dr. Christian, Professor, Zingsheimstr. 25, 53359 Rheinbach; Institut für Öffentliches Recht, Adenauerallee 24-42, 53113 Bonn, (02 28) 7379 25, Fax (02 28) 7348 69, E-Mail lshillgruber@jura.uni-bonn.de

252. Hobe, Dr. Stephan, LL.M., Universitätsprofessor, In der Asbach 32, 53347 Alfter-Impekoven, (02 28) 9489300 ; Universität zu Köln, Institut für Luft- und Weltraumrecht und Lehrstuhl für Völker- und Europarecht, europäisches und internationales Wirtschaftsrecht, Albertus-Magnus-Platz, 50923 Köln, (02 21) 47023 37, E-Mail Stephan.Hobe@uni-koeln.de 
253. Hochhuth, Dr. Martin, Privatdozent, Kaiser-Joseph-Straße 268, 79098 Freiburg;

Albert-Ludwigs-Universität Freiburg, Institut für Öffentliches Recht, Abteilung III, Staatsrecht, Platz der Alten Synagoge 1, 79085 Freiburg, (07 61) 2 03-22 43, Fax (07 61) 2 03-22 40, E-Mail hochhuth@jura.uni-freiburg.de

254. Hoffmann-Riem, Dr. Wolfgang, Professor, Richter des Bundesverfassungsgerichts a.D., Auguststr. 15, 22085 Hamburg, (0 40) 642258 48, Fax (0 40) 69645 806, E-Mail whoffmann-riem@gmx.de;

Universität Hamburg, Rothenbaumchaussee 33, 20148 Hamburg, (0 40) 428 38-30 33, Fax (0 40) 428 38-26 35

E-Mail ceri@jura.uni-hamburg.de

255. Höfling, Dr. Wolfram, M.A., Professor, Bruchweg 2, 52441 Linnich, (0 24 62) 36 16; Universität zu Köln, Institut für Staatsrecht, Albertus-Magnus-Platz, 50923 Köln, (02 21) 4 70-33 95, Fax (02 21) 4 70-50 75, E-Mail Sekretariat@institut-staatsrecht.de

256. Hofmann, Dr. Ekkehard, Professor, Koselstr. 51, 60318 Frankfurt am Main, (069) 174989 27;

Julius-Maximilians-Universität Würzburg, Institut für Staats- und Verwaltungsrecht, Rechtsphilosophie, Domerschulstr. 16, 97070 Würzburg, (0931) 31-83 738, Fax (0931) 31-82 988, E-Mail ekkehard.hofmann@jura.uni-wuerzburg.de

257. Hofmann, Dr. Dr. h. c. Hasso, o. Professor, Christoph-Mayer-Weg 5, 97082 Würzburg, (09 31) 873 88, Fax (09 31) 7832 88, oder Torstr. 176, 10115 Berlin, (0 30) 2813075 , E-Mail hasso-hofmann@gmx.de; Humboldt-Universität zu Berlin, Unter den Linden 6, 10099 Berlin, (0 30) 20 93-35 53 
258. Hofmann, Dr. Dr. Rainer, Universitätsprofessor, Bergstr. 83, 69121 Heidelberg, (0 62 21) 4010 04;

Johann-Wolfgang-Goethe-Universität Frankfurt am Main, Lehrstuhl für Öffentliches Recht, Völkerrecht und Europarecht, Senckenberganlage 31, 60325 Frankfurt am Main, (0 69) 7 98-2 53 17, Fax (0 69) 7 98-2 53 18, E-Mail R.Hofmann@jur.uni-frankfurt.de

259. Hohmann, Dr. Harald, Privatdozent, Furthwiese 10, 63654 Büdingen, (0 60 49) 9529 12, Fax (0 60 49) 9529 13;

Hohmann \& Partner Rechtsanwälte, Schloßgasse 2, 63654 Büdingen, (0 60 42) 95 67-0, Fax (0 60 42) 95 67-67, E-Mail harald.hohmann@hohmann-partner.com

260. Hollerbach, Dr. Dr. h.c. Alexander, o. Professor, Runzstraße 86, 79102 Freiburg i.Br., (07 61) 2171413

261. Holoubek, Dr. Michael, Universitätsprofessor, Zehenthofgasse 36/8, A-1190 Wien, (00 43) 131773 72, Fax (00 43) 13177372 18;

Institut für Österreichisches und Europäisches Öffentliches Recht, Wirtschaftsuniversität Wien, Althanstraße 39-45, A-1090 Wien, (00 43) 13 13 36-46 60, Fax (00 43) 1313 36-7 13, E-Mail michael.holoubek@wu.ac.at

262. Hölscheidt, Dr. Sven, Minsterialrat, apl. Professor, Westfälische Straße 45, 10711 Berlin;

Deutscher Bundestag, Fachbereich Verfassung und Verwaltung, Platz der Republik 1, 11011 Berlin, (0 30) 2 27-3 24 25/3 23 25, Fax (0 30) 2 27-3 64 71/3 62 07, E-Mail vorzimmer.wd3@bundestag.de

263. Holzinger, Dr. Gerhart, Professor, Präsident des Österreichischen Verfassungsgerichtshofs, Judenplatz 11, A-1010 Wien, (00 43) 1531224 12, Fax (00 43) 153122512 
264. Holznagel, Dr. Bernd, LL.M., Professor, Kronprinzenstraße 105, 44135 Dortmund, (02 31) 58987 06, Fax (02 31) 58987 09;

WWU Münster, Juristische Fakultät, ITM, Abt. II, Leonardo-Campus 9, 48149 Münster, (02 51) 83-3 86 41, Fax (02 51) 83-3 86 44, E-Mail holznagel@uni-muenster.de

265. Horn, Dr. Hans-Detlef, Professor, Am Heier 22, 35096 Weimar (Lahn)-Roth, (0 64 26) 9671 41, Fax (0 64 26) 9671 44;

Philipps-Universität Marburg, Fachbereich Rechtswissenschaften, Institut für Öffentliches Recht, Universitätsstr. 6, 35032 Marburg, (0 64 21) 2823810 oder 28231 26, Fax (0 64 21) 28238 39, E-Mail Hornh@staff.uni-marburg.de

266. Hösch, Dr. Ulrich, apl. Professor, RA, Kirchenstraße 72, 81675 München; Kanzlei Dr. Gronefeld, Thoma \& Kollegen, Prinzregentenplatz 23, 81675 München, (0 89) 9607 13 80, Fax (03212) 8463724 E-Mail uhoesch@juradvance.de

267. Huber, Dr. Peter M., o. Professor, Richter des Bundesverfassungsgerichts, Josef-Heppner-Str. 2, 82049 Pullach i. I., (0 89) 744246 62, Fax (0 89) 744248 52;

Universität München, Lehrstuhl für Öffentliches Recht und Staatsphilosophie,

Professor-Huber-Platz 2, 80539 München, (0 89) 21 80-35 76, Fax (0 89) 21 80-50 63, E-Mail peter.m.huber@jura.uni-muenchen.de

268. Hufeld, Dr. Ulrich, Universitätsprofessor, Stratenbarg 40a, 22393 Hamburg, (0 40) 210074 40;

Helmut-Schmidt-Universität/

Universität der Bundeswehr Hamburg, Fakultät für Wirtschafts- und Sozialwissenschaften, Professur für Öffentliches Recht und Steuerrecht, Holstenhofweg 85, 22043 Hamburg, (0 40) 65 41-28 59, Fax (0 40) 65 41-37 33, E-Mail Hufeld@hsu-hh.de 
269. Hufen, Dr. Friedhelm, o. Professor, Backhaushohl 62, 55128 Mainz, (0 61 31) 3 44 44, Fax (0 61 31) 3614 49;

Johannes-Gutenberg-Universität Mainz, Fachbereich Rechts- und Wirtschaftswissenschaften, 55099 Mainz,

(0 61 31) 39-2 2354 oder -2 30 45,Fax (0 61 31) 39-2 42 47, E-Mail hufen@uni-mainz.de

270. Huster, Dr. Stefan, Professor, Ruhr-Universität Bochum, Lehrstuhl für Öffentliches Recht II: Staats- und Verwaltungsrecht mit bes. Berücksichtigung des Sozialrechts, Universitätsstraße 150, 44780 Bochum, Gebäude GC 7/135, (02 34) 32-2 22 39, Fax (02 34) 32-1 42 71,

E-Mail stefan.huster@rub.de

271. Ibler, Dr. Martin, Professor, Lindauer Straße 3, 78464 Konstanz;

Universität Konstanz, Fachbereich Rechtswissenschaften, Postfach D 106, Universitätsstraße 10, 78457 Konstanz, (0 75 31) 88-24 80/-23 28, E-Mail Martin.Ibler@uni-konstanz.de

272. Iliopoulos-Strangas, Dr. Julia, Professorin,

A.Metaxa 2, GR-10681 Athen, (00 30) 2 10-3 826083 oder -3823344 , Fax (00 30) 2 10-3 8054 13, Mobil (00 30) 69445952 00;

Universität Athen, Juristische Fakultät, Ippokratous 33 (5. Stock), GR-10680 Athen, (00 30) 2 10-3 6884 22, E-Mail juliostr@law.uoa.gr

273. Ipsen, Dr. Jörn, o. Professor, Präsident des Niedersächsischen Staatsgerichtshofs, Luisenstr. 41, 49565 Bramsche, (0 54 61) 44 96, Fax (0 54 61) 634 62; Institut für Kommunalrecht, Universität Osnabrück, 49069 Osnabrück, (05 41) 9 69-61 69 oder -61 58, Fax (05 41) 9 69-61 70, E-Mail instkr@uos.de 
274. Ipsen, Dr. Dr. h.c. mult. Knut, o. Professor, Nevelstr. 59, 44795 Bochum, (02 34) 4312 66;

Deutsches Rotes Kreuz (DRK), Königswinterer Str. 29, 53227 Bonn

275. Isensee, Dr. Dr. h.c. Josef, o. Professor, Meckenheimer Allee 150, 53115 Bonn, (02 28) 6934 69;

Universität Bonn, Adenauerallee 24-42, 53113 Bonn, (02 28) 7358 50, Fax (02 28) 7348 69, E-Mail isensee@uni-bonn.de

276. Ismer, Dr. Roland, Professor, Werderstr. 11, 86159 Augsburg;

Lehrstuhl für Steuerrecht und Öffentliches Recht, Friedrich-Alexander-Universität Erlangen-Nürnberg, Lange Gasse 20, 90403 Nürnberg, (09 11) 53 02-3 53, Fax (09 11) 53 02-1 65, E-Mail Roland.Ismer@wiso.uni-erlangen.de

277. Jaag, Dr. Tobias, o. Professor, Bahnhofstr. 22, Postfach 2957, CH-8022 Zürich, (00 41) 4421363 63, Fax (00 41) 4421363 99;

Rechtswissenschaftliches Institut, Rämistraße 74/18, CH-8001 Zürich, (00 41) 4463430 20, Fax (00 41) 4463443 85, E-Mail tobias.jaag@rwi.uzh.ch

278. Jachmann, Dr. Monika, Universitätsprofessorin, Richterin am Bundesfinanzhof, Am Feldkreuz 2a, 82467 Garmisch-Partenkirchen, (01 72) 74044 48, E-Mail jm@jachmann.com; Bundesfinanzhof München, Ismaninger Straße 109, 81675 München, (0 89) 92 31-0, Fax (0 89) 92 31-2 01

279. Jaeckel, Dr. Liv, Privatdozentin, Augustusweg 27, 01445 Radebeul, (03 51) 56362 86, Mobil (01 70) 70654 80, E-Mail jaeckel@rz.uni-leipzig.de 
280. Jahndorf, Dr. Christian, Privatdozent, Brunnenweg 18, 48153 Münster, (02 51) 7619683 ;

Institut für Steuerrecht, Universitätsstr. 14-16, 48143 Münster, (02 51) 83227 95, Fax (02 51) 8328386 , E-Mail c.jahndorf@fides-treuhand.de

281. Janko, Dr. Andreas, Univ.-Prof., Schwindstraße 4, A-4040 Linz/Auhof;

Institut für Staatsrecht und Politische Wissenschaften, Johannes Kepler Universität Linz, Altenberger Straße 69, A-4040 Linz/Auhof, (00 43) 732246884 56, Fax (00 43) 732246889 01, E-Mail andreas.janko@jku.at oder Elisabeth.Kamptner@jku.at

282. Janssen, Dr. Albert, apl. Professor, Landtagsdirektor i.R., Langelinienwall 16, 31134 Hildesheim, (0 51 21) 1311 12;

E-Mail a.a.janssen@t-online.de

283. Janz, Dr. Norbert, Privatdozent, Koch-Gotha-Str. 10, 18055 Rostock, (0381) 87729313 ,

Landesrechnungshof Brandenburg,

Dortusstr. 30-34, 14467 Potsdam

(03 31) 8 66-85 35, Fax (03 31) 8 66-85 18,

E-Mail janz@uni-potsdam.de

284. Jarass, Dr. Hans D., LL.M. (Harvard), o. Professor, Baumhofstr. 37 d, 44799 Bochum, (02 34) 7720 25;

Institut für Umwelt- und Planungsrecht, Universität Münster, Universitätsstr. 14-16, 48143 Münster, (02 51) 83297 93, Fax (02 51) 83292 97, E-Mail jarass@uni-muenster.de

285. Jestaedt, Dr. Matthias, Professor, Röntgenstraße 12a, 91080 Uttenreuth, (0 91 31) 8146 846;

Albert-Ludwigs-Universität, Rechtswissenschaftliche Fakultät, 79085 Freiburg i. Br., (0761) 203978 00, Fax (0761) 20397802

E-Mail rechtstheorie@jura.uni-freiburg.de 
286. Jouanjan, Dr. Olivier, Professor,

32, rue de Vieux Marché aux Poissons, F-97000 Strasbourg, (00 33) 6613325 59;

Université de Strasbourg,

Institut de Recherches Carré de Malberg,

11, rue du Maréchal Juin B.P. 68, F-67046 Strasbourg Cedex, (00 33) 3881430 34;

Albert-Ludwigs-Universität, Rechtswissenschaftliche Fakultät, Institut für öffentliches Recht (Abt. 2),

Platz der Alten Synagoge, 79085 Freiburg i. Br.,

E-Mail olivier.jouanjan@unistra.fr

287. Jochum, Dr. Georg, Professor,

Oberhofstraße 92, 88045 Friedrichshafen,

(01 70) 23867 58;

Zeppelin University, Lehrstuhl für Europarecht \& Internationales Recht der Regulierung,

Maybachplatz 5, 88045 Friedrichshafen,

(0 75 41) 600914 81, Fax (0 75 41) 600914 99,

E-Mail Georg.Jochum@zeppelin-university.de

288. Jochum, Dr. jur. Heike, Mag. rer. publ., Professorin, Buchsweilerstraße 77, 66953 Pirmasens;

Institut für Finanz- und Steuerrecht

an der Universität Osnabrück,

Martinistraße 10, 49080 Osnabrück,

(05 41) 9 69-61 68 (Sek.), -61 61 (direkt),

Fax (05 41) 9 69-61 67,

E-Mail Heike.Jochum@gmx.net

289. Kadelbach, Dr. Stefan, LL.M., Professor,

Goethe-Universität, Institut für Öffentliches Recht,

Lehrstuhl für Öffentliches Recht,

Europarecht und Völkerrecht,

Grüneburgplatz 1, 60323 Frankfurt am Main,

(0 69) 798 34295, Fax (0 69) 79834516 ,

E-Mail s.kadelbach@jur.uni-frankfurt.de

290. Kägi-Diener, Dr. Regula, Professorin, Rechtsanwältin, Marktgasse 14, CH-9004 St. Gallen, (00 41) 71-2 2381 21, Fax (00 41) 71-2 2381 28, E-Mail switzerland@ewla.org oder regula.kaegi-diener@ewla.org 
291. Kahl, Dr. Arno, Privatdozent, Lärchenstraße 4a, A-6063 Rum, (00 43) 5 12-26 55 00;

Universität Innsbruck, Institut für Öffentliches Recht, Finanzrecht und Politikwissenschaft, Innrain 82, A-6020 Innsbruck, (00 43) 51250782 04, Fax (00 43) 51250727 48, E-Mailarno.kahl@uibk.ac.at

292. Kahl, Dr. Wolfgang, M.A., o. Professor, Albert-Schweitzer-Straße 2, 95447 Bayreuth, (09 21) 15092 87;

Universität Heidelberg, Institut für deutsches und europäisches Verwaltungsrecht, Friedrich-Ebert-Anlage 6-10, 69117 Heidelberg, (0 62 21) 5474 28, Fax (0 62 21) 5477 43, E-Mailkahl@jurs.uni-heidelberg.de

293. Kaltenborn, Dr. Markus, Universitätsprofessor, Neue Tremoniastr. 30, 44137 Dortmund, (02 31) 18159 09; Ruhr-Universität Bochum, Juristische Fakultät 44780 Bochum, (02 34) 32-2 5252 oder -2 52 63, Fax (02 34) 32-1 44 21, E-Mail markus.kaltenborn@ruhr-uni-bochum.de

294. Kämmerer, Dr. Jörn Axel, Professor, Am Kaiserkai 53, 20457 Hamburg, (0 40) 480922 23;

Bucerius Law School, Hochschule für Rechtswissenschaft, Jungiusstraße 6, 20335 Hamburg, (0 40) 307 06-1 90, Fax (0 40) 3070 6-1 95, E-Mail axel.kaemmerer@law-school.de

295. Karpen, Dr. Ulrich, Professor, Ringstr. 181, 22145 Hamburg, (0 40) 67783 98, E-Mail ulrich.karpen@yahoo.de; Universität Hamburg, Schlüterstr. 28, 20146 Hamburg, (0 40) $42838-3023$ oder -4514 od. -4555 
296. Kästner, Dr. Karl-Hermann, o. Professor, Alt-Rathausstr. 5, 72511 Bingen, (0 75 71) 32 23, Fax (0 75 71) 32 12;

Universität Tübingen, Juristische Fakultät, Geschwister-Scholl-Platz, 72074 Tübingen, (0 70 71) 29729 71, Fax (0 70 71) 2950 96, E-Mail Kaestner@jura.uni-tuebingen.de

297. Kaufmann, Dr. Christine, Professorin, Lehrstuhl für Staats- und Verwaltungsrecht, Völker- und Europarecht, Universität Zürich, Rämistrasse 74/5, CH-8001 Zürich, (00 41) 4463448 65, Fax (00 41) 4463443 78, E-Mail Lst.kaufmann@rwi.uzh.ch

298. Kaufmann, Dr. Marcel, Privatdozent, Rechtsanwalt, Senefelderstraße 7, 10437 Berlin; Freshfields Bruckhaus Deringer, Environment, Planning and Regulatory (EPR), Potsdamer Platz 1, 10785 Berlin, (0 30) 202 83-8 57(Sekretariat), (0 30) 202 83-6 00, Fax (0 30) 202 83-7 66, E-Mail marcel.kaufmann@freshfields.com

299. Keller, Dr. Helen, Professorin,

Eigenstraße 16, CH-8008 Zürich, (00 41) 4442223 20;

Universität Zürich, Rechtswissenschaftliches Institut, Rämistraße 74, CH-8001 Zürich, (00 41) 4463436 89, Fax (00 41) 4463443 39, E-Mail Helen.keller@rwi.unizh.ch

300. Kempen, Dr. Bernhard, o. Professor, Rheinblick 1, 53424 Remagen/Oberwinter, (0 22 28) 9132 91, Fax (0 22 28) 9132 93; Institut für Völkerrecht und ausländisches öffentliches Recht, Universität zu Köln, Gottfried-Keller-Straße 2, 50931 Köln, (02 21) 47023 64, Fax (02 21) 47049 92, E-Mail Bernhard.Kempen@uni-koeln.de 
301. Kersten, Dr. Jens, Professor, Hoheneckstr. 28, 81243 München, (089) 954793 40;

Ludwig-Maximilians-Universität München, Lehrstuhl für Öffentliches Recht und Verwaltungswissenschaften, Professor-Huber-Platz 2, 80539 München, (0 89) 21 80-21 13, Fax (0 89) 21 80-1 35 15, E-Mail jens.kersten@jura.uni-muenchen.de

302. Khakzadeh-Leiler, Dr. Lamiss, ao. Univ.-Professorin, Mähderweg 37 c, A-6841 Mäder, (0043) 6764338765 ;

Universität Innsbruck, Institut für Öffentliches Recht, Staats- und Verwaltungslehre, Innrain $52 \mathrm{~d}, \mathrm{~A}-6020$ Innsbruck, (0043) 507-8232, Fax (0043) 507-2828,

E-Mail lamiss.khakzadeh@uibk.ac.at

303. Khan, Dr. Daniel-Erasmus, Professor, Institut für Öffentliches Recht und Völkerrecht Universität der Bundeswehr München, Werner-Heisenberg-Weg 39, 85579 Neubiberg (0 89) 60 04-46 90 oder -4262 oder -2048 , Fax (0 89) 60 04-46 91, E-Mail Khan@unibw.de

304. Kilian, Dr. Michael, Professor, Am Burgwall 15, 06198 Brachwitz; Juristische Fakultät, Universität Halle-Wittenberg, Universitätsplatz 3-5, Juridicum, 06099 Halle (Saale), (03 45) 55-2 31 70, Fax (03 45) 55-2 72 69, E-Mail Kilian@jura.uni-halle.de

305. Kingreen, Dr. Thorsten, Professor, Agnes-Miegel-Weg 10, 93055 Regensburg, (09 41) 70402 41;

Lehrstuhl für Öffentliches Recht, Sozialrecht und Gesundheitsrecht, Universität Regensburg, Universitätsstr. 31, 93053 Regensburg, (09 41) 9432607 od. 26 08, Fax (09 41) 94336 34, E-Mail king@jura.uni-regensburg.de 
306. Kirchhof, Dr. Ferdinand, o. Professor, Walther-Rathenau-Str. 28, 72766 Reutlingen, (0 71 21) 4902 81, Fax (0 71 21) 4794 47; Universität Tübingen, Juristische Fakultät, Geschwister-Scholl-Platz, 72074 Tübingen, (0 70 71) 2 97-25 61 oder -81 18, Fax (0 70 71) 2943 58, E-Mail Kirchhof@uni-tuebingen.de

307. Kirchhof, Dr. Gregor, LL.M., Universitätsprofessor, Fasanenstr. 12, 85591 Vaterstetten, (08106) 34809 34;

Ludwig-Maximilians-Universität München, Institut für Politik und Öffentliches Recht, Professor-Huber-Platz 2, 80539 München, (089) 218050 75,

E-Mail mail@gregorkirchhhof.de

308. Kirchhof, Dr. Dr. h.c. mult. Paul, o. Professor, Am Pferchelhang 33/1, 69118 Heidelberg, (0 62 21) 8014 47; Universität Heidelberg, Friedrich-Ebert-Anlage 6-10, 69117 Heidelberg, (0 62 21) 5474 57, E-Mail paul.kirchhof@paul-kirchhof.de

309. Kirn, Dr. Michael, o. Professor, Rummelsburgerstr. 3, 22147 Hamburg, (0 40) 64738 43;

Universität der Bundeswehr, Institut für Öffentliches Recht, Postfach 7008 22, 22043 Hamburg, (0 40) 65 41-27 82 oder (0 40) 65 41-2590

310. Kirste, Dr. Stephan, Professor, Am Gutleuthofhang 18, 69118 Heidelberg, (0 62 21) 804503 Fax (0 62 21) 8045 03; Andrássy Gyula Deutschsprachige Universität, Fakultät für Vergleichende Staats- und Rechtswissenschaft, Professur für Öffentliches Recht, Europarecht und Rechtsphilosophie, H-1088 Budapest, Pollack Mihály tér 3, (+36) 1266 4408-137, Fax (+36) 1266 3099, E-Mail kirste.stephan@andrassyuni.hu 
311. Kischel, Dr. Uwe, LL.M. (Yale), Attorney-at-law (New York), o. Professor, Dorfstraße 34, 17121 Düvier, (03 9998$) 315$ 46;

Ernst-Moritz-Arndt-Universität Greifswald, Domstr. 20a, 17489 Greifswald, (0 38 34) 86-21 80, Fax (0 38 34) 86-21 82, E-Mail kischel@uni-greifswald.de

312. Klaushofer, Dr. Reinhard, az. Prof., Universität Salzburg, Kapitelgasse 5-7, 5020 Salzburg, 0043 (0) 662 8044-3634, Fax 0043 (0) 662 8044-303, E-Mail reinhard.klaushofer@sbg.ac.at

313. Klein, Dr. Eckart, Universitätsprofessor, Heideweg 45, 14482 Potsdam, (03 31) 7058 47;

Lehrstuhl für Staatsrecht, Völkerrecht und Europarecht, Universität Potsdam, August-Bebel-Str. 89, 14482 Potsdam, (03 31) 9 77-35 16, oder-35 11, Fax (03 31) 9 77-32 24, E-Mail Klein@rz.uni-potsdam.de

314. Klein, Dr. Hans Hugo, Universitätsprofessor, Heilbrunnstr. 4, 76327 Pfinztal, (0 72 40) 73 00, E-Mail hanshklein@web.de

315. Kley, Dr. Andreas, Professor, Stallikerstr. 10a, CH-8142 Uitikon Waldegg; Rechtswissenschaftliches Institut, Rämistr. 74/34, CH-8001 Zürich, (0041) 44-634 50 20, Fax (0041) 44-634 5029 E-Mail andreas.kley@bluewin.ch

316. Kloepfer, Dr. Michael, o. Professor, Taubertstraße 19, 14193 Berlin, (0 30) 82524 90, Fax (0 30) 82526 90; Institut für Öffentliches Recht und Völkerrecht, Humboldt-Universität zu Berlin, Unter den Linden 9-11 (Palais), 10099 Berlin, (0 30) 20 93-33 40 oder -33 31, Fax (0 30) 20 93-34 38, E-Mail Michael.Kloepfer@rewi.hu-berlin.de 
317. Kluth, Dr. Winfried, Professor, Blumenstr. 17, 06108 Halle (Saale), (03 45) 29085 10;

Martin-Luther-Universität Halle-Wittenberg, Juristische Fakultät, Universitätsplatz 3-5, 06108 Halle, Raum: Juridicum 1.13, 06099 Halle (Saale),

(03 45) 55232 23, Fax (03 45) 55272 93,

E-Mail winfried.kluth@jura.uni-halle.de

318. Kment, Dr. Martin, LL.M. (Cambridge), Professor, Mergelberg 89, 48161 Münster, (02 51) 2953 54;

EBS Universität für Wirtschaft und Recht, HEUSSEN-Stiftungslehrstuhl für Öffentliches Recht und Europarecht, Recht der erneuerbaren Energien sowie Umwelt- und Planungsrecht, Gustav-Stresemann-Ring 3, 65189 Wiesbaden, (0611) 7102 2234, Fax (0611) 7102102234 , E-Mail martin.kment@ebs.edu

319. Knauff, Dr. Matthias, LL.M. Eur., Professor, Am Scheuerberg 5, 97222 Rimpar, (0 93 65) 8890 57;

EBS Universität für Wirtschaft und Recht, Lehrstuhl für Öffentliches Recht, insbesondere deutsches und europäisches öffentliches Wirtschaftsrecht, Gustav-Stresemann-Ring 3, 65189 Wiesbaden, (0611) 7102 2237, Fax (0611) 710210 2237, Email matthias.knauff@ebs.edu

320. Kneihs, Dr. Benjamin, Univ. Professor,

Niederland 73, A-5091 Unken, Österreich; Universität Salzburg, Fachbereich öffentliches Recht, Kapitelgasse 5-7, A-5020 Salzburg, (00 43) 66280 44-36 11, Fax (00 43) 66280 44-3 03, E-Mail benjamin.kneihs@sbg.ac.at

321. Knemeyer, Dr. Franz-Ludwig, o. Professor, Unterdürrbacher Str. 353, 97080 Würzburg, (09 31) 961 18;

Universität Würzburg,

Domerschulerstr.16, 97070 Würzburg, (09 31) 31-8 28 99, Fax (09 31) 31-23 17, E-Mail aseubert@jura.uni-wuerzburg.de 
322. Knies, Dr. Wolfgang, o. Professor, Am Botanischen Garten 5, 66123 Saarbrücken, (06 81) 3998 88, Fax (06 81) 3998 88;

Universität Saarbrücken, Postfach 1511 50, 66041 Saarbrücken, (06 81) 3 02-31 58,

E-Mail w.knies@mx.uni-saarland.de

323. Knöpfle, Dr. Franz, em. Professor, Höhenweg 22, 86391 Stadtbergen;

Universität Augsburg, Universitätsstr. 2, 86159 Augsburg, (08 21) 5 98-46 59, Fax (08 21) 5 98-45 47

324. Koch, Dr. Hans-Joachim, Professor, Wendlohstr. 80, 22459 Hamburg, (0 40) 55188 04, Fax (0 40) 55188 04; Universität Hamburg, Fakultät für Rechtswissenschaft, Edmund-Siemers-Allee 1, 20146 Hamburg, (0 40) 428 38-39 77 oder -54 43, Fax (0 40) 428 38-62 80, E-Mail hans-joachim.koch@jura.uni-hamburg.de

325. Koch, Dr. Thorsten, Privatdozent, Emanuel-Geibel-Str. 4, 49143 Bissendorf-Schledehausen, (0 54 02) 77 74;

Institut für Kommunalrecht Universität Osnabrück, Martinistr. 12, 49069 Osnabrück, (0 54 1) 9 69-61 69, Fax (0 54 1) 9 69-61 64, E-Mail tkoch@uos.de

326. Köck, Dr. Wolfgang, Professor, UFZ-Umweltforschungszentrum Leipzig-Halle $\mathrm{GmbH}$, Permoserstraße 15, 04318 Leipzig; Universität Leipzig, Lehrstuhl für Umweltrecht, Postfach 1009 20, 04009 Leipzig, (03 41) 2 35-31 40, Fax (03 41) 2 35-28 25, E-Mail Wolfgang.Koeck@ufz.de

327. Koenig, Dr. Christian, LL.M. (London), Universitätsprofessor, Zentrum für Europäische Integrationsforschung, Rheinische Friedrich-Wilhelms-Universität, Walter-Flex-Str. 3, 53113 Bonn, (02 28) 73-18-91/-92/-95, Fax (02 28) 73-18 93, E-Mail sekretariat.zeia@uni-bonn.de 
328. Kokott, Dr. Juliane, LL.M. (Am. Un.), S.J.D. (Harvard), Universitätsprofessorin, Generalanwältin, (0 62 21) 45 16-17;

Gerichtshof der Europäischen Gemeinschaften, Th. More 2214, Bd. Konrad Adenauer, L-2925, Luxemburg, (0 03 52) 430322 21, E-Mail juliane.kokott@curia.europa.eu

329. Kolonovits, Dr. Dieter, Mag., M.C.J., ao. Univ.-Professor, Berggasse 17/41 A-1090 Wien, (00 43) 699192028 95;

Universität Wien, Institut für Staats- und Verwaltungsrecht, Schottenbastei 10-16, A-1010 Wien (Juridicum), (00 43) 14277354 16, Fax (00 43) 14277354 19, E-Mail dieter.kolonovits@univie.ac.at

330. König, Dr. Doris, Professorin, Bucerius Law School, Hochschule für Rechtswissenschaft, Jungiusstr. 6, 20355 Hamburg, (0 40) 307 06-2 01 Fax (0 40) 307 06-1 90, E-Mail doris.koenig@law-school.de

331. König, Dr. Dr. Klaus, Universitätsprofessor, Albrecht-Dürer-Str. 20, 67346 Speyer, (0 62 32) 2902 16;

Deutsche Hochschule für Verwaltungswissenschaften Speyer, Postfach 14 09, 67324 Speyer, (0 62 32) 6 54-3 69 oder -350 oder -3 55, Fax 06232654 306, E-Mail k.koenig@dhv-speyer.de

332. Kopetzki, DDr. Christian, Universitätsprofessor, Institut für Staats- und Verwaltungsrecht, Medizienrecht, Universität Wien, Schottenbastei 10-16, A-1010 Wien, (00 43) 14277354 11, Fax (00 43) 14277354 19, E-Mail christian.kopetzki@univie.ac.at

333. Korinek, Dr. Dr. h.c. Karl, o. Professor, Präsident des Österreichischen Verfassungsgerichtshofs a.D., Auhofstr. 225-227, A-1130 Wien, (00 43) 187648 76;

Institut für Staats- und Verwaltungsrecht, Universität Wien, Schottenbastei 10-16, A-1010 Wien, (00 43) 142 77-3 54 42, Fax (00 43) 142 77-3 5449 
334. Korioth, Dr. Stefan, Professor, Institut für Politik und Öffentliches Recht der Universität München, Professor-Huber-Platz 2/III, 80539 München, (0 89) 21 80-27 37, Fax (0 89) 21 80-39 90, E-Mail Korioth@jura.uni-muenchen.de

335. Kotulla, Dr. Michael, M.A., Professor, Universität Bielefeld, Fakultät für Rechtswissenschaft, Postfach 1001 31, 33501 Bielefeld, (05 21) 1 06-25 00, Fax (05 21) 1 06-80 91, E-Mail Michael.Kotulla@uni-bielefeld.de

336. Kotzur, Dr. Markus, LL.M. (Duke Univ.), o. Professor, Thomasgasse 4, 04109 Leipzig, (03 41) 22487 96;

Lehrstuhl für Europarecht, Völkerrecht, Öffentliches Recht, Universität Leipzig, Burgstr. 27, 04109 Leipzig, (03 41) 97-35 2 10, Fax (03 41) 97-3 52 19, E-Mail kotzur@rz.uni-leipzig.de

337. Krajewski, Dr. Markus, Professor, Frommannstr. 19, 90419 Nürnberg, Friedrich-Alexander-Universität Erlangen-Nürnberg, Fachbereich Rechtswissenschaft, Schillerstr. 1, 91054 Erlangen, (09131) 85222 60, Fax (09131) 85269 50, E-Mail markus.krajewski@jura.uni-erlangen.de

338. Krause, Dr. Peter, o. Professor, Weinbergstr. 12, 54317 Korlingen, (0 65 88) 73 33;

Universität Trier, 54286 Trier, (06 51) 2 01-25 87, Fax (06 51) 2 01-38 03, E-Mail Krausepe@uni-trier.de

339. Krausnick, Dr. Daniel, Privatdozent,

Bubenreutherstr. 19 b, 91094 Bräuningshof, (09133) 6048 60;

Friedrich-Alexander-Universität Erlangen-Nürnberg, Institut für Staats- und Verwaltungsrecht, Schillerstr. 1, 91054 Erlangen, (09131) 85230 26, Fax (09131) 85263 82, E-Mail Daniel.Krausnick@jura.uni-erlangen.de 
340. Krawietz, Dr. Werner, o. Professor, Nienbergweg 29, 48161 Münster, (02 51) 8614 51;

Lehrstuhl für Rechtssoziologie, Universität Münster, Bispinghof 24-25, 48143 Münster, (02 51) 8325 91, E-Mail Krawiet@uni-muenster.de

341. Krebs, Dr. Walter, Professor, Waldmannstr. 19, 12247 Berlin, (0 30) 77107 58, Fax (0 30) 77107 58;

Freie Universität Berlin, Boltzmannstr. 4, 14195 Berlin, (0 30) 8 38-59 21, Fax (0 30) 8 38-59 22, E-Mail lehrstuhl-krebs@fu-berlin.de

342. Kreßel, Dr. Eckhard, Professor, Körschtalstr. 21, 73760 Ostfildern, (09 31) 313 05;

Juristische Fakultät der Universität Würzburg, Domerschulstr. 16, 97070 Würzburg, E-Mail eckhard.kressel@daimler.com

343. Krieger, Dr. Heike, Professorin, Freie Universität Berlin, Fachbereich Rechtswissenschaft, Van't-Hoff-Straße 8, 14195 Berlin, (0 30) 83851453 , E-Mail hkrieger@zedat.fu-berlin.de

344. Kriele, Dr. Martin, o. Professor, Dorf 11, A-6900 Möggers, (00 43) 55 73-8 24 96, Fax (00 43) 55 73-8 24 97; Universität Köln, Albertus-Magnus-Platz 1, 50923 Köln, (02 21) 4 70-22 30, Fax (02 21) 4 70-50 10

345. Kröger, Dr. Klaus, Universitätsprofessor, Hölderlinweg 14, 35396 Gießen, (06 41) 522 40; Universität Gießen, 35394 Gießen, (06 41) 99 23-1 30, Fax (06 41) 99 23-0 59 
346. Krugmann, Dr. Michael, Privatdozent, Stellaustieg 3, 22143 Hamburg, (0 40) 67788 60, Fax (0 40) 67788 60, E-Maildr@michaelkrugmann.de

347. Kube, Dr. Hanno, LL.M. (Cornell), Universitätsprofessor, Am Langenstück 23, 65343 Eltville, (0 61 23) 79578 48;

Lehrstuhl für Öffentliches Recht und Steuerrecht, Fachbereich Rechts- und Wirtschaftswissenschaften, Johannes Gutenberg-Universität Mainz, Jakob Welder-Weg 9, Zimmernr. 03.214, 55099 Mainz, (0 61 31) $3922725 / 3923043$ (Sekr.), Fax (0 61 31) 39238 26, E-Mail hkube@uni-mainz.de

348. Kucsko-Stadlmayer, Dr. Gabriele, Ao. Universitätsprofessorin, Rooseveltplatz 4-5, A-1090 Wien, (00 43) 140838 59;

Universität Wien, Institut für Staats- und Verwaltungsrecht, Schottenbastei 10-16, A-1010 Wien, (00 43) 14277354 18, Fax (00 43) 1427793 54, E-Mail gabriele.kucsko-stadlmayer@univie.ac.at

349. Kugelmann, Dr. Dieter, Professor, Am Klosterwald 24, 48165 Münster, DieterKugelmann@web.de; Deutsche Hochschule der Polizei, Zum Roten Berge 18-24, 48165 Münster, (0 25 01) 8 06-4 37, -2 79 (Sekr.), -5 25 (Fax), E-Mail dieter.kugelmann@dhpol.de

350. Kühling, Dr. Jürgen, LL.M. (Brüssel), Universitätsprofessor, Kellerweg 12 b, 93053 Regensburg, (09 41) 70560 79; Universität Regensburg, Lehrstuhl für Öffentliches Recht und Immobilienrecht, Universitätsstr. 31, 93053 Regensburg, (09 41) 9 43-60 60, Fax (09 41) 9 43-60 62, E-Mail juergen.kuehling@jura.uni-regensburg.de 
351. Kühne, Dr. Jörg-Detlef, Professor, Münchhausenstr. 2, 30625 Hannover, (05 11) 5565 63;

Universität Hannover, Königsworther Platz 1, 30167 Hannover, (05 11) 7 62-81 48, Fax (05 11) 7 62-82 28, E-Mail Kuehne@oera.uni-hannover.de

352. Kunig, Dr. Philip, Professor, Freie Universität Berlin, Institut für Staatslehre, Boltzmannstraße 3, 14195 Berlin, (0 30) 83853 0-10, Fax (0 30) 83853 0-11, E-Mail Kunig@zedat.fu-berlin.de

353. Küpper, Dr. Herbert, Professor, Arcostr. 1, 80333 München; Institut für Ostrecht, Landshuter Str. 4, 93047 Regensburg, (09 41) 94354 50, Fax (09 41) 94354 65, E-Mail Herbert.Kuepper@ostrecht.de

354. Lachmayer, Dr. Konrad, Privatdozent, Weitlofgasse 12/10, 1180 Wien; Universität Wien, Rechtswissenschaftliche Fakultät, Institut für Staats- und Verwaltungsrecht, Schottenbastei 10-16, 1010 Wien, (0043) 14277 35471, Fax (0043) 14277 35479, E-Mail konrad.lachmayer@univie.ac.at

355. Ladeur, Dr. Karl-Heinz, Professor, Universität Hamburg, Fakultät für Rechtswissenschaft, Schlüterstraße 28, 20146 Hamburg, (0 40) 428 38-57 52, Fax (0 40) 428 38-26 35, E-Mail karl-heinz.ladeur@jura.uni-hamburg.de

356. Lang, Dr. Heinrich, Professor, Dipl.-Sozialpädagoge, Nikolausstraße 48, 51149 Köln;

Universität Rostock, Lehrstuhl für Öffentliches Recht, Sozial- u. Gesundheitsrecht, Möllner Straße 10, 18109 Rostock, (03 81) 4 98-81 70, Fax (03 81) 4 98-81 72, E-Mail lehrstuhl.lang@uni-rostock.de 
357. Lange, Dr. Klaus, Universitätsprofessor,

Lilienweg 22, 35423 Lich,

(0 64 04) 56 81;

Universität Gießen, Fachbereich Rechtswissenschaften,

Hein-Heckroth-Straße 5, 35390 Gießen, (06 41) 992 11-80 oder -81, Fax (06 41) 992 11-89,

E-Mail Klaus.Lange@recht.uni-giessen.de

358. Langenfeld, Dr. Christine, Professorin,

Schillerweg 34, 04155 Leipzig,

E-Mail Dr.Langenfeld@t-online.de;

Juristisches Seminar der Georg-August-Universität,

Platz der Göttinger Sieben 6, 37073 Göttingen,

(05 51) 39-73 84, Fax (05 51) 39-1 23 92,

E-Mail enomiko@gwdg.de

359. Laskowski, Dr. Silke Ruth, Professorin,

Gertigstraße 13, 22303 Hamburg,

(0 40) 3666 15, Fax (0 40) 3666 15, Mobil (01 79) 23156 63,

E-Mail Silke.Laskowski@jura.uni-hamburg.de;

Universität Kassel, Institut für Wirtschaftsrecht - FB 07,

Nora-Platiel-Str. 5, 34109 Kassel, (05 61) 80428 74,

E-Mail s-laskowski@uni-kassel.de

360. Laubinger, Dr. Hans-Werner, M.C.L., Professor,

Philipp-Wasserburg-Str. 45, 55122 Mainz,

(0 61 31) 431 91;

Universität Mainz, 55099 Mainz,

(0 61 31) 3959 42,

E-Mail Laubinger@mail.jura.uni-mainz.de

361. Laurer, Dr. Hans René, a.o. Universitätsprofessor,

Scheffergasse 27a, A-2340 Mödling,

(00 43) 2636204 02;

Wirtschafts-Universität,

Augasse 2-6, A-1190 Wien,

(00 43) 131336 oder 4669 oder 4158

362. Lecheler, Dr. Helmut, o. Professor,

Freie Universität Berlin, Fachbereich Rechtswissenschaft,

Institut für Völkerrecht, Europarecht und ausl. öffentl. Recht,

Vant'-Hoff-Str. 8, 14195 Berlin,

(030)7 73-63 17, Fax (030)7 73-58 23

E-Mail mail@lecheler.eu 
363. Lege, Dr. Joachim, Professor, Fischstr. 19, 17489 Greifswald, (0 38 34) 7739 41;

Rechts- und Staatswissenschaftliche Fakultät, Ernst-Moritz-Arndt-Universität, Domstr. 20, 17489 Greifswald, (0 38 34) 86-21 50, Fax (0 38 34) 86-21 56, E-Mail lege@uni-greifswald.de

364. Lehner, Dr. Moris, Universitätsprofessor, Kaiserplatz 7, 80803 München, (0 89) 340206 46;

Ludwig-Maximilians-Universität, Lehrstuhl für Öffentliches Recht, insbesondere öffentliches Wirtschaftsrecht und Steuerrecht, Ludwigstr. 28 (Rgb.), 80539 München, (0 89) 218027 18, Fax (0 89) 3335 66, E-Mail Moris.Lehner@jura.uni-muenchen.de

365. Leisner, Dr. mult. Dr. h.c. Walter, o. Professor, Pienzenauerstr. 99, 81925 München, (0 89) 9894 05, Fax (0 89) 982909 97; Universität Erlangen, Schillerstr. 1, 91054 Erlangen, (0 91 31) 852260

366. Leisner, Dr. Walter Georg, Privatdozent, Knöbelstr. 16, 80538 München, (0 89) 9894 24;

Universität Hamburg, Fakultät für Rechtswissenschaft, Schlüterstr. 28, 20146 Hamburg, E-Mail leisner@1z-legal.de

367. Leisner-Egensperger, Dr. Anna, Universitätsprofessorin, Bachmairstrasse 15, 81243 München-Pasing; (089) 88909 356, Mobil (0173) 39241 45; Lehrstuhl für Öffentliches Recht und Steuerrecht, Friedrich-Schiller-Universität Jena, Carl-Zeiss-Straße 3, 07743 Jena, (0 36 41) 9422 51, Fax (0 36 41) 9422 52, E-Mail A.Leisner@recht.uni-jena.de 
368. Leitl-Staudinger, Dr. Barbara, Universitätsprofessorin, Hohe Straße 135, A-4040 Linz;

Institut für Fernunterricht in den Rechtswissenschaften, Johannes Kepler Universität Linz, Petrinumstraße 12, A-4040 Linz, (00 43) 732246819 00, Fax (00 43) 732246819 10, E-Mail barbara.leitl-staudinger@jku.at

369. Lenze, Dr. Anne, Privatdozentin, Sandstraße 19, 64625 Bensheim, (0 62 51) 5808 52;

Fachhochschule Darmstadt, Adelungstraße 51, 64283 Darmstadt, (0 61 51) 1689 65, Fax (0 61 51) 1689 90, E-Mail anne.lenze@t-online.de

370. Lepsius, Dr. Oliver, LL.M. (Chicago), Professor, Romanstr. 53, 80639 München, (0 89) 14332482 und (0921) 150844 30;

Lehrstuhl für Öffentliches Recht, Allgemeine und Vergleichende Staatslehre, Universität Bayreuth, Universitätsstr. 30, 95440 Bayreuth, (09 21) 55-62 61, (09 21) 55-62 62, E-Mail oliver.lepsius@uni-bayreuth.de

371. Lerche, Dr. Dr. h. c. Peter, o. Professor, Junkersstr. 13, 82131 Gauting, (0 89) 85020 88, Fax (0 89) 8502088

372. Lewinski, Dr. Kai von, Privatdozent, Bismarckstr. 70, 10627 Berlin, (030) 373055 13;

Humboldt-Universität zu Berlin, Juristische Fakultät, Unter den Linden 9, 10099 Berlin, (030) 2093-3331; Fax (030) 209320213 341, E-Mail kai.lewinski@rewi.hu-berlin.de

373. Lienbacher, Dr. Georg, Universitätsprofessor, Obere Donaustr. 43/2/44, A-1020 Wien; Institut für Österreichisches und Europäisches Öffentliches Recht, Wirtschaftsuniversität Wien, Althanstraße 39-45 (UZA 3), A-1090 Wien, (00 43) 1313 36-54 02, Fax (00 43) 1313 36-92 22, E-Mail Georg.Lienbacher@wu.ac.at 
374. Lindner, Dr. Josef Franz, Privatdozent, Großhaderner Straße 14 b, 81375 München, (0 89) 7032 45, Fax (0 89) 740093 85, E-Mail josef.lindner@stmwfk.bayern.de

375. Link, Dr. jur. Dres. theol. h.c. Heinz-Christoph, em. Professor, Rühlstraße 35, 91054 Erlangen, (0 91 31) 2093 35, Fax (0 91 31) 5345 66; Hans-Liermann-Institut für Kirchenrecht, Hindenburgstr. 34, 91054 Erlangen, (0 91 31) 85228 25, Fax (0 91 31) 8524064

376. Lipphardt, Dr. Hanns-Rudolf, apl. Professor, Auf der Weide 7, 69126 Heidelberg, (0 62 21) 3823 12; Universität Heidelberg, 69120 Heidelberg, (0 62 21) 4111 98, Fax (0 62 21) 400675

377. Listl, Dr. Joseph, o. Professor, Jesuitengemeinschaft Pedro Arrupe, Bibergerstr. 8, 82008 Unterhaching; dienstlich (stets für die Post benutzen!): Institut für Staatskirchenrecht der Diözesen Deutschlands, Adenauerallee 19, 53111 Bonn

378. Lorenz, Dr. Dieter, o. Professor, Bohlstr. 21, 78465 Konstanz, (0 75 33) 68 22; Universität Konstanz, Postfach 5560 D 100, 78434 Konstanz, (0 75 31) 882530 , E-Mail Dieter.Lorenz@uni-konstanz.de

379. Lorz, Dr. Ralph Alexander, LL.M. (Harvard), Attorney-at-Law (New York), Universitätsprofessor, Eickelscheidt 8 a, 40883 Ratingen, (01 70) 41218 66;

Universität Düsseldorf, Lehrstuhl für deutsches und ausländisches öffentliches Recht, Völker- und Europarecht, Universitätsstraße 1, 40225 Düsseldorf, (02 11) 8 11-14 35, Fax (02 11) 8 11-14 56, E-Mail Al.Lorz@uni-duesseldorf.de 
380. Losch, Dr. Dr. Bernhard, Professor, Dürerstr. 9, 42119 Wuppertal, (02 02$) 423525$

381. Loschelder, Dr. Wolfgang, Professor, Sonnenlandstr. 5, 14471 Potsdam, (03 31) 9736 80, Fax (03 31) 95119 95;

Universität Potsdam, August-Bebel-Str. 89, Haus 1, 14482 Potsdam, (03 31) 9 77-34 12

382. Löwer, Dr. Wolfgang, Professor, Hobsweg 15, 53125 Bonn, (02 28) 2506 92, Fax (02 28) 2504 14; Universität Bonn, Adenauerallee 24-42, 53113 Bonn, (02 28) 7392 78/73 92 80, Fax (02 28) 7339 57, E-Mail w.loewer@uni-bonn.de

383. Lübbe-Wolff, Dr. Gertrude, Professorin, (05 21) 8826 59;

Universität Bielefeld, Fakultät Rechtswissenschaft, Universitätsstr. 25, Postfach 100131, 33615 Bielefeld, (05 21) 1 06-43 86, Fax (05 21) 1 06-80 85, E-Mail Gertrude.Luebbe-Wolff@uni-bielefeld.de

384. Luchterhandt, Dr. Otto, Professor, Im Wendischen Dorfe 28, 21335 Lüneburg, (0 41 31) 2329 65, Fax (0 41 31) 2329 65; Universität Hamburg, Schlüterstr. 28 (Rechtshaus), 20146 Hamburg, (0 40) 428 38-45 62, E-Mail ottolucht@arcor.de

385. Lühmann, Dr. Hans, Privatdozent, Pannebäcker Str. 7a, 40593 Düsseldorf, (02 11) 2399534

386. Luther, Dr. Jörg, Professor

Via Roero di Cortanze 2, I-10124 Torino, (0039) 011835 607, Dipartimento POLIS Via Cavour 84, I-15100 Alessandria, (0039) 0131283 745, Fax (0039) 0131283704 luther@sp.unipmn.it 
387. Mächler, Dr. iur. August, Professor, Schindellegistrasse 15, CH-8808 Pfäffikon, (00 41) 5541043 20;

Sicherheitsdepartement des Kt. Schwyz, Postfach 1200, 6431 Schwyz, (00 41) 4181920 02, Fax (00 41) 4181920 19, E-Mail august-maechler@swissonline.ch

388. Magen, Dr. Stefan, M.A., Professor, Kallenweg 6, 53129 Bonn, (0228) 909176 79;

Ruhr-Universität Bochum, Lehrstuhl für Öffentliches Recht, Rechtsphilosophie und Rechtsökonomik, Universitätsstr. 150, 44780 Bochum, (0234) 32 -22 809, Fax (0234) $32-14327$ E-Mail magen@rub.de

389. Mager, Dr. Ute, Universitätsprofessorin, Universität Heidelberg, Juristische Fakultät, Friedrich-Ebert-Anlage 6-10, 69117 Heidelberg, (0 62 21) 547737 oder (01 71) 55400 78, E-Mail ute.mager@jurs.uni-heidelberg.de

390. Magiera, Dr. Siegfried, Universitätsprofessor, Feuerbachstr. 1, 67354 Römerberg, (0 6232$) 848$ 98;

Deutsche Hochschule für Verwaltungswissenschaften Speyer, Freiherr-vom-Stein-Str. 2, 67346 Speyer, (0 62 32) 6543 48, E-Mail s.magiera@dhv-speyer.de

391. Mahlmann, Dr. Matthias, Professor, Rindermarkt 7, CH-8001 Zürich; Lehrstuhl für Rechtstheorie, Rechtssoziologie und Internationales Öffentliches Recht, Universität Zürich, Treichlerstr. 10, CH-8032 Zürich, (00 41) 4463415 87, Fax (00 41) 4463443 91, E-Mail matthias.mahlmann@rwi.uzh.ch

392. Majer, Dr. jur. utr. Diemut, Rechtsanwältin, Universitätsprof., Universität Bern;

Welfenstr. 35, 76137 Karlsruhe, (07 21) 816650 Fax (07 21) 8176 63, E-Mail majer@kanzlei-karlstr62.de 
393. Mangoldt, Dr. Hans von, Professor,

Goetheweg 1, 72147 Nehren,

(0 74 73) 79 08;

Universität Tübingen, Juristische Fakultät,

Geschwister-Scholl-Platz, 72074 Tübingen,

(0 70 71) 2973302

394. Mann, Dr. Thomas, Professor,

Im Torfveen 19, 46147 Oberhausen,

(02 08) 6754 98;

Juristisches Seminar der Georg-August-Universität Göttingen,

Platz der Göttinger Sieben 6, 37073 Göttingen,

(05 51) 39-47 23, Fax (05 51) 39-79 78,

E-Mail sekretariatmann@jura.uni-goettingen.de

395. Manssen, Dr. Gerrit, Universitätsprofessor,

Konrad-Adenauer-Allee 15, 93051 Regensburg,

(09 41) 928 45;

Juristische Fakultät, Universität Regensburg,

93040 Regensburg,

(09 41) 9 43-32 55, Fax (09 41) 9 43-32 57,

E-Mail Gerrit.Manssen@jura.uni-regensburg.de

396. Mantl, Dr. Dr. h.c. Wolfgang, em. o. Universitätsprofessor,

Wiener Str. 256/XI/33, A-8051 Graz,

(00 43) 3 16-68 13 06;

Institut für Österreichisches, Europäisches und Vergleichendes

Öffentliches Recht,

Politikwissenschaft und Verwaltungslehre,

Karl-Franzens-Universität Graz,

Universitätsstr. 15/K3, A-8010 Graz,

(00 43) 3 16-3 8033 70,

E-Mail wolfgang.mant1@uni-graz.at

397. Marauhn, Dr. Thilo, M.Phil., Professor,

An der Fels 20, 35435 Wettenberg,

(06 41) 8773275 , Fax (06 41) 8773275 ,

E-Mail thilo.marauhn@recht.uni-giessen.de;

Professur für Öffentliches Recht, Völkerrecht

und Europarecht, Justus-Liebig-Universität Gießen,

Licher Straße 76, 35394 Gießen,

(06 41) 99211 50/51, Fax (06 41) 99211 59,

E-Mail intlaw@recht.uni-giessen.de 
398. Marko, Dr. Joseph, o. Professor, Kasernstr. 35, A-8010 Graz, (00 43) 3 16-46 22 38;

Institute of Austrian, European and Comparative Public Law and Political Sciences, University of Graz, Universitätsstraße 15/B4, A-8010 Graz, (00 43) 3163 80-33 74, Fax (00 43) 3163 80-94 52, E-Mail josef.marko@uni-graz.at

399. Marti, Dr. Arnold, Titularprofessor der Universität Zürich, Fernsichtstraße 5, CH-8200 Schaffhausen, (00 41) 52-6 2418 10, E-Mail a.g.marti@swissonline.ch;

Obergericht des Kantons Schaffhausen, Frauengasse 17, CH-8200 Schaffhausen, (00 41) 52-6 3274 24, Fax (00 41) 52-6 3278 36, E-Mail arnold.marti@ktsh.ch

400. Martínez Soria, Dr. José, Privatdozent, Braschzeile 9, 14109 Berlin, (0 30) 80586766 ; Institut für Völkerrecht der Universität Göttingen, Platz der Göttinger Sieben 5, 37073 Göttingen, (05 51) 3921 97, Fax (05 51) 3921 96, E-Mail jmartin@gwdg.de

401. Martini, Dr. Mario, Professor, Adams-Lehmann-Str. 8, 80797 München, (0 89) 320026 10;

Deutsche Hochschule für Verwaltungswissenschaften Speyer, Lehrstuhl für Verwaltungswissenschaft, Freiherr-vom-Stein-Straße 2, 67346 Speyer, (0 62 32) 6 54-33 8, Fax (0 62 32) 6 54-40 4, E-Mail martini@dhv-speyer.de

402. März, Dr. Wolfgang, Professor, Zelckstraße 1, 18055 Rostock, (03 81) 37792 55, Fax (03 81) 37792 56; Lehrstuhl für Öffentliches Recht und Verfassungsgeschichte, Universität Rostock, Möllner Str. 10, 18109 Rostock, (03 81) 49881 90, Fax (03 81) 4988002 , E-Mail wolfgang.maerz@uni-rostock.de 
403. Masing, Dr. Johannes, Professor, Richter des Bundesverfassungsgerichts, Schlossbezirk 3, 76131 Karlsruhe; Albert-Ludwigs-Universität Freiburg, Platz der Alten Synagoge, 79085 Freiburg, (07 61) 2 03-22 52, Fax (07 61) 20322 93, E-Mail johannes.masing@jura.uni-freiburg.de

404. Mastronardi, Dr. Philippe Andrea, Professor, Stadlstrasse $2 \mathrm{CH}-6048$ Horw, (00 41) 4134027 67, Fax (00 41) 413408 54;

Universität St. Gallen, Rechtswissenschaftliche Abteilung, Bodanstr. 3, CH-9000 St. Gallen, (00 41) 7122423 34, Fax (00 41) 7122439 08, E-Mail Philippe.Mastronardi@unisg.ch

405. Maurer, Dr. Hartmut, o. Professor, Säntisblick 10, 78465 Konstanz, (0 75 33) 13 12;

Universität Konstanz, 78464 Konstanz, (0 75 31) 8836 57, Fax (0 75 31) 8831 96, E-Mail hartmut.maurer@uni-konstanz.de

406. Mayer, Dr. Franz, LL.M. (Yale), Universitätsprofessor, Universität Bielefeld, Lehrstuhl für Öffentliches Recht, Europarecht, Völkerrecht, Rechtsvergleichung und Rechtspolitik, Postfach 1001 31, 33501 Bielefeld, (05 21) 1 06-44 12, Fax (05 21) 1 06-8 90 16, E-Mail franz.mayer@uni-bielefeld.de

407. Mayer-Tasch, Dr. Peter Cornelius, Professor, Am Seeberg 13, 86938 Schondorf, (0 81 92) 86 68;

Hochschule für Politik München, Ludwigstr. 8, 80539 München, (0 89) 28803 99-0, Fax (0 89) 28803 99-22

408. Meessen, Dr. Karl Matthias, Professor, Rotterdamer Straße 45, 40474 Düsseldorf, (02 11) 15942 11, Fax (02 11) 15942 12, E-Mail Karl.Meessen@t-online.de 
409. Mehde, Dr. Veith, Mag.rer.publ., Professor, Lilienstr. 23, 30167 Hannover, (05 11) 89829 06;

Lehrstuhl für Öffentliches Recht, insbesondere Verwaltungsrecht, Leibniz Universität Hannover, Königsworter Platz 1, 30167 Hannover, (05 11) 7 62-82 06, Sekr.: -82 07, Fax (05 11) 7 62-1 91 06, E-Mail mehde@jura.uni-hannover.de

410. Meng, Dr. Werner, Universitätsprofessor, Im Herrengarten 8, 55263 Wackernheim, (0 6132$) 9770$ 65;

Europa-Institut, Universität des Saarlandes, Postfach 1511 50, 66041 Saarbrücken, (06 81) 30266 60, Fax (06 81) 30266 62, E-Mail w.meng@kabelmail.de

411. Menzel, Dr. Jörg, Privatdozent, The Senate of the Kingdom of Cambodia, Chamcar Morn, State Palace, Norodom Blvd., Phnom Penh, Cambodia, (0 08 55) 123335 28, Fax (0 08 55) 232114 46, E-Mail joergmenzel@gmx.net

412. Merli, Dr. Franz, Universitätsprofessor, Institut für Österreichisches, Europäisches und Vergleichendes Öffentliches Recht, Politikwissenschaft und Verwaltungslehre, Karl-Franzens-Universität Graz, Universitätsstraße 15, A-8010 Graz, (00 43) 3163 80-36 02, Fax (00 43) 3163 80-94 50, E-Mail merli@uni-graz.at

413. Merten, Dr. Dr. Detlef, o. Professor, Von-Dalberg-Str. 8, 67487 St. Martin, (0 63 23) 18 75;

Deutsche Hochschule für Verwaltungswissenschaften Speyer, Freiherr-vom-Stein-Str. 2-6, 67346 Speyer, (0 62 32) 6 54-3 49; oder -3 30, E-Mail Merten@dhv-speyer.de 
414. Meßerschmidt, Dr. Klaus, Privatdozent, Hynspergstr. 29, 60322 Frankfurt am Main, (0 69) 554587 ;

University of Latvia, EuroFaculty, Raina bulv. 19, LV-1586 Riga/Lettland, (00 371) 78202 78, Fax (00 371) 78202 60, E-Mail Messerschmidtk1@aol.com

415. Meyer, Dr. Dr. h. c. Hans, Professor, Georg-Speyer-Str. 28, 60487 Frankfurt am Main, (0 69) 770129 26, Fax (0 69) 770129 27;

Humboldt-Universität zu Berlin, Juristische Fakultät, Unter den Linden 6, 10099 Berlin, (0 30) 20 93-35 28 (Sekr.) oder -33 47, Fax (0 30) 20 93-27 29, E-Mail Hans.Meyer@rewi.hu-berlin.de

416. Meyer, Dr. Stephan, Privatdozent, Friedrich-Engels-Straße 62, 99086 Erfurt, (0163) 2900 557;

Universität Erfurt, Nordhäuser Str. 63, 99089 Erfurt, (0361)737-4762, Fax (0361) 737-4709, E-Mail stephan.meyer@uni-erfurt.de

417. Meyn, Dr. Karl-Ulrich, Professor, Leyer Str. 36, 49076 Osnabrück, (05 41) 1264 82;

Universität Jena, Schillerhaus, Schillergässchen 2, 07745 Jena, (0 36 41) 9311 85, Fax (0 36 41) 9311 87, E-Mail karl-ulrich.meyn@t-online.de

418. Michael, Dr. Lothar, Professor, Niederkasseler Kirchweg 124, 40547 Düsseldorf; Professur für Öffentliches Recht, Universitätsstraße 1, Geb. 24.91, 40225 Düsseldorf, (02 11) 81114 12, E-Mail Lothar.Michael@uni-duesseldorf.de 
419. Möllers, Dr. Christoph, LL.M., Professor, Zehdenicker Str. 14, 10119 Berlin;

Humboldt-Universität zu Berlin, Lehrstuhl für Öffentliches Recht, insbesondere Verfassungsrecht, und Rechtsphilosophie, Unter den Linden 6, 10099 Berlin, (0 30) 20 93-35 85, Fax (0 30) 20 93-35 52, E-Mail sekretariat.moellers@rewi.hu-berlin.de

420. Morgenthaler, Dr. Gerd, Professor, Tilsiter Str. 33, 57250 Netphen; Universität Siegen, Fachbereich 5, Hölderlinstr. 3, 57068 Siegen, (02 71) 74024 02, E-Mail morgenthaler@recht.wiwi.uni-siegen.de

421. Morlok, Dr. Martin, Professor, Poßbergweg 51, 40629 Düsseldorf, (02 11) 2868 68;

Heinrich-Heine-Universität, Juristische Fakultät, Universitätsstr. 1, Gebäude 24.91, 40225 Düsseldorf, (02 11) 81153 51, Fax (02 11) 8111460 , E-Mail 1s.morlok@uni-duesseldorf.de

422. Morscher, Dr. Siegbert, em. Universitätsprofessor, Tschiggyfreystr. 11a, A-6020 Innsbruck, (00 43) 5 12-28 62 10;

Leopold-Franzens-Universität, Institut für Öffentliches Recht, Staats- und Verwaltungslehre, Innrain 52d, A-6020 Innsbruck, (00 43) 5 12-5 078210 oder -11, Fax (00 43) 5 12-5 072828 , E-Mail siegbert.morscher@uibk.ac.at

423. Möstl, Dr. Markus, Professor, Birkenstraße 77, 95447 Bayreuth, (09 21) 50717 68, E-Mail Markus.Moestl@t-online.de; Rechts- und Wirtschaftswissenschaftliche Fakultät Universitätsstr. 30, 95440 Bayreuth, (09 21) 55-28 66, Fax (09 21) 5520 41, E-Mail markus.moestl@uni-bayreuth.de 
424. Muckel, Dr. Stefan, Universitätsprofessor,

Ringstraße 122, 42929 Wermelskirchen,

(0 21 93) 5310 74;

Universität zu Köln, Institut für Kirchenrecht, 50923 Köln, (02 21) 4 70-37 77 oder 4 70-26 79, E-Mail Kirchenrecht@uni-koeln.de

425. Mückl, Dr. Stefan, apl. Professor, Adenauerallee 129, 53115 Bonn;

Albert-Ludwigs-Universität Freiburg i.Br., Institut für Öffentliches Recht IV, Platz der Universität 3, 79085 Freiburg i.Br., (07 61) 2 03-22 64, Fax (07 61) 2 03-22 97, E-Mail s.mueck1@gmx.de

426. Müller, Dr. Bernhard, Privatdozent, Lisseeweg 36/2, A-1210 Wien, (0043) 676934 934-3, E-Mail b_mueller@tele2.at; Dorda Brugger Jordis Rechtsanwälte $\mathrm{GmbH}$, Dr.-Karl-Lueger-Ring 10, A-1010 Wien, (0043) 15334795 57, Fax (0043) 15334795 5057, E-Mail bernhard.mueller@dbj.at

427. Müller, Dr. Dr. h.c. Georg, o. Professor em., Sugenreben 10, CH-5018 Erlinsbach, (00 41) 6284438 73, Fax (00 41) 628444204 E-Mail georg-mueller@sunrise.ch

428. Müller, Dr. Dr. h.c. Jörg Paul, o. Professor em., Universität Bern, Kappelenring 42a, $\mathrm{CH}-3032$ Hinterkappelen bei Bern, (00 41) 319010570 , E-Mail jpmueller@bluewin.ch

429. Müller-Franken, Dr. Sebastian, Professor, Schützenstr. 1c, 35039 Marburg/Lahn, (0 64 21) 2070340 oder

Westermühlstr. 28, 80469 München, (0 89) 202398 28;

Professur für Öffentliches Recht, Philipps-Universität Marburg, Universitätsstraße 6, 35032 Marburg/Lahn, (0 64 21) 28231 22, Fax (0 64 21) 28238 40, E-Mail mueller-franken@staff.uni-marburg.de 
430. Müller-Terpitz, Dr. Ralf, Professor, Albrecht-Dürer-Str. 11, 40489 Düsseldorf, (02 03) 72818 97, Fax (02 03) 72818 98;

Universität Passau, Lehrstuhl für Staats- und Verwaltungsrecht sowie Wirtschaftsverwaltungs-, Medien- und Informationsrecht, Innstr. 40 (Nikolakloster), 94032 Passau, (08 51) 50922 20, Fax (08 51) 50922 22, E-Mail mueller-terpitz@uni-passau.de

431. Münch, Dr. Dr. h.c. Ingo von, Professor, Hochrad 9, 22605 Hamburg, (0 40) 8296 24, Fax (0 40) 823449

432. Murswiek, Dr. Dietrich, o. Professor, Lindenaustr. 17, 79199 Kirchzarten, (0 76 61) 992 37; Institut für Öffentliches Recht, Universität Freiburg, 79085 Freiburg,

(07 61) 2 03-22 37 oder -41, Fax (07 61) 2 03-22 40, E-Mail Dietrich.Murswiek@jura.uni-freiburg.de

433. Musil, Dr. Andreas, Professor, Mendelssohn-Bartholdy-Str. 34, 14480 Potsdam, (03 31) 74534 53;

Universität Potsdam, Lehrstuhl für Öffentliches Recht, insbesondere Verwaltungs- und Steuerrecht, August-Bebel-Str 89, 14482 Potsdam, (03 31) 97732 33,

E-Mail musil@uni-potsdam.de

434. Mußgnug, Dr. Reinhard, o. Professor, Keplerstr. 40, 69120 Heidelberg, (0 62 21) 4362 22, Fax (0 62 21) 4083 09;

Universität Heidelberg, Institut für Finanz- und Steuerrecht, Friedrich-Ebert-Anlage 6-10, 69117 Heidelberg, (0 62 21) 5474 66, Fax (0 62 21) 5476 54, E-Mail Reinhard.Mussgnug@urz.uni-heidelberg.de

435. Mutius, Dr. Albert von, o. Professor, Hof „Frankenthaler Moor“, Poseritz-Ausbau Nr. 8, 18574 Poseritz auf Rügen, (03 83 07) 405 99, Mobil (01 76) 218205 81, Fax (03 83 07) 403 49, E-Mail avm.law@gmx.de 
436. Nettesheim, Dr. Martin, o. Professor, Horemer 13, 72076 Tübingen, (0 70 71) 2546 04;

Universität Tübingen, Juristische Fakultät, Geschwister-Scholl-Platz, 72074 Tübingen, (0 70 71) 29725 60, Fax (0 70 71) 2958 47, E-Mail Nettesheim@jura.uni-tuebingen.de

437. Neumann, Dr. Volker, Professor, Neckarstaden 10, 69117 Heidelberg, (0 62 21) 1612 66;

Humboldt-Universität zu Berlin, Lehrstuhl für

Öffentliches Recht, Sozialrecht und Staatstheorie, Unter den Linden 6, 10099 Berlin, (0 30) 209334 60, Fax (0 30) 209334 52,

E-Mail volker.neumann@rewi.hu-berlin.de

438. Nicolaysen, Dr. Gert, Professor, Bockhorst 68a, 22589 Hamburg, (0 40) 87017 47;

Universität Hamburg, Seminar für Öffentliches Recht und Staatslehre, Abteilung Europarecht, Schlüterstraße 28, 20146 Hamburg, E-Mail gert.nicolaysen@jura.uni-hamburg.de

439. Niedobitek, Dr. Matthias, Universitätsprofessor, Weststr. 99, 09116 Chemnitz, (0371) 275504 18;

Professur für Europäische Integration mit dem Schwerpunkt Europäische Verwaltung, Technische Universität Chemnitz, Thüringer Weg 9, 09126 Chemnitz, (03 71) 5 31-3 49 12, E-Mail matthias.niedobitek@phil.tu-chemnitz.de

440. Nierhaus, Dr. Michael, Professor, Am Moosberg 1c, 50997 Köln, (0 22 36) 636 29, Fax (0 22 36) 9637 95, E-Mail michael@nierhaus.org 
441. Nolte, Dr. Georg, Professor, Ansbacher Str. 73, 10777 Berlin, (0 30) 536741 92;

Institut für Völker- und Europarecht, Humboldt-Universität zu Berlin, Unter den Linden 6, 10099 Berlin, (0 30) 20 93-33 49, Fax (0 30) 20 93-33 84, E-Mail georg.nolte@rewi.hu-berlin.de

442. Nolte, Dr. Martin, Privatdozent, Düppelstraße 1, 24105 Kiel, (04 31) 5658 22, Fax (04 31) 5658 22;

Christian-Albrechts-Universität zu Kiel, Lehrstuhl für Öffentliches Recht und Verwaltungswissenschaften, Olshausenstraße 40, 24098 Kiel, (04 31) 8 80-45 46, Fax (04 31) 8 80-45 82, E-Mail drnolte@lvstein.uni-kiel.de

443. Novak, Dr. Richard, o. Professor, Thadd. Stammel-Str. 8, A-8020 Graz, (00 43) 3 16-5 35 16; Universität (00 43) 3 16-3 80-33 71, E-Mail richard.novak@uni-graz.at

444. Nowak, Dr. Carsten, Universitätsprofessor, Jevenstedter Str. 69g, 22547 Hamburg, (0 40) 88003 17;

Lehrstuhl für Öffentliches Recht, insb. Europarecht, Europa-Universität Viadrina Frankfurt (Oder), Große Scharrnstr. 59, 15230 Frankfurt (Oder), (03 35) 55 34-27 10, -27 11, Fax (03 35) 55 34-7 27 11, E-Mail cnowak@euv-frankfurt-o.de

445. Nußberger, Dr. Angelika, Professorin, Eichenhainallee 15, 51427 Bergisch Gladbach; Institut für Ostrecht an der Universität zu Köln, Klosterstr. 79 d, 50931 Köln, (02 21) 47055 83, Fax (02 21) 47055 82, E-Mail angelika.nussberger@uni-koeln.de 
446. Odendahl, Dr. Kerstin, Professorin,

Goethestrasse 43, CH-9008 St. Gallen;

Christian-Albrechts-Universität zu Kiel,

Walther-Schücking-Institut für Internationales Recht,

Westring 400, 24118 Kiel,

(04 31) 880-2149, Fax (04 31) 880-1619,

E-Mail odendahl@internat-recht.uni-kiel.de

447. Oebbecke, Dr. Janbernd, Universitätsprofessor,

Huberstr. 13a, 48155 Münster,

(02 51) 23051 70;

Kommunalwissenschaftliches Institut,

Universität Münster,

Universitätsstr. 14-16, 48143 Münster,

(02 51) 83-2 18 06, Fax (02 51) 83-2 18 33,

E-Mail oebbecke@uni-muenster.de

448. Oeter, Dr. Stefan, Professor,

Wulfsdorfer Weg 122, 22359 Hamburg,

(0 40) 609519 57;

Universität Hamburg,

Institut für Internationale Angelegenheiten,

Rothenbaumchaussee 33, 20148 Hamburg,

(0 40) 4283845 65, Fax (0 40) 4283862 62,

E-Mail S-Oeter@jura.uni-hamburg.de

449. Ohler, Dr. Christoph, LL.M., Professor,

Berghoffsweg 4, 07743 Jena,

(0 36 41) 2070 81;

Rechtswissenschaftliche Fakultät, Friedrich-Schiller-Universität Jena, Carl-Zeiß-Str. 3, 07743 Jena

(0 36 41) 9422 60, Fax (0 36 41) 9422 62,

E-Mail christoph.ohler@recht.uni-jena.de

450. Öhlinger, Dr. Theo, o. Universitätsprofessor,

Tolstojgasse 5/6, A-1130 Wien,

(00 43-1) 87712 60;

Universität Wien, Schottenbastei 10-16, A-1010 Wien,

E-Mail theodor.oehlinger@univie.ac.at 
451. Oldiges, Dr. Martin, Universitätsprofessor, August-Bebel-Straße 31, 04275 Leipzig, (03 41) 21192 33, Fax (03 41) 14968 16, E-Mail martin@oldiges.de;

Universität Leipzig, Juristenfakultät, Otto-Schill-Str. 2, 04109 Leipzig, (03 41) 973 51 31, Fax (03 41) 9735139

452. Oppermann, Dr. Dres. h.c. Thomas, o. Professor, Burgholzweg 122, 72070 Tübingen, (0 70 71) 495 33, Fax (0 70 71) 447 02, E-Mail oppermannprof@aol.com; Universität Tübingen, Juristische Fakultät, Geschwister-Scholl-Platz, 72074 Tübingen, (0 70 71) 29725 58, Fax (0 70 71) 2958 47, E-Mail Thomas.Oppermann@uni-tuebingen.de

453. Ossenbühl, Dr. Fritz, Professor, Im Wingert 12, 53340 Meckenheim, (0 22 25) 174 82;

Universität Bonn, 53113 Bonn, (02 28) 73 55-72 oder -73

454. Osterloh, Dr. Lerke, Professorin, Richterin des Bundesverfassungsgerichts a.D., Institut für Öffentliches Recht, Universität Frankfurt, Postfach 1119 32, 60054 Frankfurt am Main, (0 69) 79822711 oder 286 11, Fax (0 69) 798225 62, E-Mail Osterloh@jur.uni-frankfurt.de

455. Pabel, Dr. Katharina, Professorin, Scherzergasse 10/8, A-1020 Wien (00 43) 1212530 2;

Johannes Kepler Universität Linz, Institut für Verwaltungsrecht und Verwaltungslehre, Altenberger Straße 69, A-4040 Linz, (00 43) (0) 732 / 2468-8490, Fax (00 43) (0) 732/2468-8489 E-Mail katharina.pabel@jku.at

456. Pabst, Dr. Heinz-Joachim, Privatdozent, Universität zu Köln, Prüfungsamt der Rechtswissenschaftlichen Fakultät, Albertus-Magnus-Platz, 50923 Köln, (02 21) 4 70-57 99, Fax (02 21) 4 70-67 22, E-Mail hpabst@uni-koeln.de 
457. Pache, Dr. Eckhard, Professor, Hauptstraße 82, 97218 Gerbrunn;

Julius-Maximilians-Universität Würzburg, Domerschulstraße 16, 97070 Würzburg, (09 31) 31-8 23 09, Fax (0931) 31-23 19, E-Mail pache@jura.uni-wuerzburg.de

458. Papier, Dr. Dr. h.c. Hans-Jürgen, em. o. Professor, Präsident des Bundesverfassungsgerichts a.D., Institut für Politik und Öffentliches Recht, Universität München,

Professor-Huber-Platz 2, 80539 München, (0 89) 21 80-62 94 oder -62 95, Fax (0 89) 218031 99, E-Mail LS.Papier@jura.uni-muenchen.de

459. Paulus, Dr. Andreas, Professor, Hermann-Föge-Weg 17, 37073 Göttingen; Institut für Völkerrecht und Europarecht, Platz der Göttinger Sieben 5, 37073 Göttingen, (05 51) 3947 51, Fax (05 51) 3947 67, E-Mail apaulus@jura.uni-goettingen.de

460. Pauly, Dr. Walter, o. Professor, Lehrstuhl für Öffentliches Recht, Rechts- und Verfassungsgeschichte, Rechtsphilosophie, Universität Jena, Carl-Zeiss-Str. 3, 07743 Jena, (0 36 41) 942230 oder -31, Fax (0 36 41) 9422 32, E-Mail W.Pauly@recht.uni-jena.de

461. Pechstein, Dr. Matthias, Universitätsprofessor, Lindenallee 40, 14050 Berlin, (0 30) 30194 17, Fax (0 30) 30194 17; Jean-Monnet-Institut für Öffentliches Recht und Europarecht, Europa-Universität Viadrina Frankfurt (Oder), Große Scharrnstr. 59, 15230 Frankfurt (Oder), (03 35) 55 34-27 61, Fax (03 35) 55 34-27 69, E-Mail Pechstein@euv-frankfurt-o.de 
462. Peine, Dr. jur. Dr. h.c. Franz-Joseph, Professor, Kurpromenade 56, 14089 Berlin-Kladow, (0 30) 36561 93, Fax (0 30) 36561 93;

Lehrstuhl für Öffentliches Recht, insbesondere Verwaltungsrecht, Europa-Universität Viadrina Frankfurt (Oder), Große Scharrnstr. 59, 15230 Frankfurt (Oder), (03 35) 55 34-25 28, Fax (03 35) 55 34-25 69, E-Mail fjpeineberlin@t-online.de

463. Pernice, Dr. Ingolf, Universitätsprofessor, Laehrstraße 17a, 14165 Berlin, (0 30) 847236 15, Fax (0 30) 845091 62;

Lehrstuhl für Öffentliches Recht, Völker- und Europarecht, Humboldt-Universität zu Berlin, Unter den Linden 6, 10099 Berlin, (0 30) 20 93-34 40, Fax (0 30) 20 93-34 49, E-Mail ingolf.pernice@rz.hu-berlin.de

464. Pestalozza, Dr. Christian Graf von, Universitätsprofessor (em.), Freie Universität Berlin, Institut für Staatslehre, Staats-und Verwaltungsrecht, Dienstanschrift: Van't-Hoff-Str. 8, 14195 Berlin (Dahlem), Postanschrift: Bayernallee 12, 14052 Berlin (Westend), (0 30) 3046329 oder 838530 14, Fax (0 30) 308131 04, E-Mail c.pestalozza@fu-berlin.de

465. Peters, Dr. Anne, LL.M., Professorin, Bollwerkstr. 134, CH-4102 Binningen; Lehrstuhl für Völker- und Staatsrecht, Universität Basel, Maiengasse 51, CH-4056 Basel, (00 41) 6126725 65, Fax (00 41) 6126725 71, E-Mail Anne.Peters@unibas.ch

466. Pielow, Dr. Johann-Christian, Professor, Stiepeler Str. 96, 44801 Bochum, (02 34) 746 33;

Ruhr-Universität Bochum, Fakultät für Wirtschaftswissenschaft

- Recht der Wirtschaft -, Universitätsstr. 150, 44780 Bochum, (02 34) 3225 72 34, Fax (02 34) 32140 74, E-Mail christian.pielow@ruhr-uni-bochum.de 
467. Pieper, Dr. Stefan Ulrich, apl. Professor,

Bundespräsidialamt,

Spreeweg 1, 10557 Berlin,

(0 18 88) 50021 20, Fax (0 30) 20 00-19 99,

E-Mail stefan.pieper@bpra.bund.de

468. Pieroth, Dr. Bodo, Professor,

Gluckweg 19, 48147 Münster,

(02 51) 2332 91, Fax (02 51) 2332 94;

Institut für Öffentliches Recht und Politik, Universität Münster,

Wilmergasse 28, 48143 Münster,

(02 51) 5104 90, Fax (02 51) 5 10 49-19,

E-Mail pieroth@uni-muenster.de

469. Pietzcker, Dr. Jost, Professor,

Hausdorffstr. 95, 53129 Bonn,

(02 28) 2339 54;

Universität Bonn, 53113 Bonn,

(02 28) 739177 ,

E-Mail Pietzcker@jura.uni-bonn.de

470. Pirson, Dr. Dr. Dietrich, o. Professor,

Brunnenanger 15, 82418 Seehausen,

(0 88 41) 478 68;

Universität München,

Professor-Huber-Platz 2, 80539 München,

(0 89) 21 80-27 15,

E-Mail d.pirson@gmx.de

471. Pitschas, Dr. Rainer, o. Universitätsprofessor,

Hermann-Jürgens-Str. 8, 76829 Landau-Godramstein,

(0 63 41) 9693 81, Fax (0 63 41) 9693 82;

Deutsche Hochschule für Verwaltungswissenschaften Speyer,

Postfach 14 09, 67324 Speyer,

(0 62 32) 6 54-3 45, Fax (0 62 32) 6 54-3 05,

E-Mail rpitschas@dhv-speyer.de

472. Pöcker, Dr. Markus, Privatdozent, Zollhofstr. 4, 67061 Ludwigshafen, Johann-Wolfgang-Goethe-Universität,

Lehrstuhl Prof. Dr. G. Hermes,

Senckenberganlage 31, 60325 Frankfurt am Main, (069) 79823864 ,

E-Mail poecker@jur.uni-frankfurt.de 
473. Poscher, Dr. Ralf, Universitätsprofessor, Zasiusstr. 6, 79102 Freiburg, (0761) 6124191 ;

Albert-Ludwigs-Universität Freiburg, Institut für Staatswissenschaft und Rechtsphilosophie, Abt. 2: Rechtsphilosophie, Platz der Alten Synagoge 1, 79085 Freiburg, (0761) 20397 570; Fax (0761) 20397571

E-Mail rechtsphilosophie@jura.uni-freiburg.de

474. Pöschl, Dr., Magdalena, Univ.-Prof., Klosterwiesgasse 31, A-8010 Graz; Universität Graz, Institut für Österreichisches, Europäisches und Vergleichendes Öffentliches Recht, Politikwissenschaft und Verwaltungslehre, Universitätsstr. 15/D3, A-8010 Graz, (00 43) 3163 80-67 07, Fax (00 43) 3163 80-94 50, E-Mail magdalena.poeschl@uni-graz.at

475. Potacs, Dr. Michael, Professor, Hammerschmidtgasse 5/3/2, A-1190 Wien, (00 43-1) 32466 23;

Institut für Österreichisches und Europäisches Öffentliches Recht, Wirtschaftsuniversität Wien, Althanstraße 39-45, A-1090 Wien, (00 43) 13133646 69, E-Mail michael.potacs@wu.ac.at

476. Preu $\beta$, Dr. Ulrich K., Professor, Friedbergstraße 47, 14057 Berlin, (0 30) 308194 33;

Hertie School of Governance, Schlossplatz 1, 10178 Berlin, (0 30) 2123123 10, Fax (0 30) 2123129 99, E-Mail ukpreuss@hertie-school.org

477. Proel $\beta$, Dr. Alexander, Professor, Heikendorfer Weg 35, 24149 Kiel (0431) 5846501 Walther-Schücking-Institut für Internationales Recht Christian-Albrechts-Universität zu Kiel Westring 400, 24098 Kiel (0431) 880-2042, Fax (0431) 880-1619 E-Mail aproelss@internat-recht.uni-kiel.de 
478. Puhl, Dr. Thomas, o. Professor, In der Aue 26a, 69118 Heidelberg, (0 62 21) 8036 64, Fax (0 62 21) 8036 69;

Universität Mannheim, Fakultät für Rechtswissenschaft, Schloss - Westflügel (W 226), 68131 Mannheim, (06 21) 1 81-13 45/-13 55, Fax (06 21) 18113 61, E-Mail puhl@staffmail.uni-mannheim.de

479. Pünder, Dr. Hermann, LL.M (Iowa), Universitätsprofessor, Hagedornstraße 25, 20149 Hamburg, (0 40) 414669 34;

Bucerius Law School, Lehrstuhl für Öffentliches Recht (einschließlich Europarecht), Verwaltungswissenschaft und Rechtsvergleichung, Postfach 3010 30, 20304 Hamburg, (0 40) 3070 6-2 60, Fax (0 40) 307 06-2 35, E-Mail hermann.puender@law-school.de

480. Puttler, Dr. Adelheid, LL.M. (University of Chicago), diplomée de l'E.N.A., Universitätsprofessorin, Lehrstuhl für Öffentliches Recht, insbesondere Europarecht, Völkerrecht und Internationales Wirtschaftsrecht, Ruhr-Universität Bochum, 44780 Bochum, (02 34) 32228 20, Fax (02 34) 32141 39, E-Mail LS-Puttler@Ruhr-Uni-Bochum.de

481. Püttner, Dr. Dr. h.c. Günter, o. Professor, Schwerdstraße 3, 67346 Speyer, (0 62 32) 71997

482. Ramsauer, Dr. Ulrich, Professor, VRiOVG, Wiesenstraße 5, 20255 Hamburg, (0 40) 431812 53;

Universität Hamburg, Fakultät für Rechtswissenschaft, Seminar für Verwaltungslehre, Rothenbaumchaussee 33, 20148 Hamburg, (0 40) 428 38-49 65, Fax (0 40) 428 38-56 70, E-Mail ulrich.ramsauer@uni-hamburg.de

483. Randelzhofer, Dr. Albrecht, o. Professor, Wulffstr. 12, 12165 Berlin, (0 30) 7926085 
484. Raschauer, Dr. Bernhard, o. Universitätsprofessor, Pfeilgasse $7 / 2 / 6$, A-1080 Wien, (00 43-1) 40833 53;

Universität Wien, Schottenbastei 10-16, A-1010 Wien, (00 43-1) 42 77-3 53 52, Fax (00 43-1) 42 77-3 54 59, E-Mail sekretariat.raschauer.staatsrecht@univie.ac.at

485. Raschauer, Dr. Nicolas, Universitätsprofessor, Kaltnergasse 7, A-5020 Salzburg, (00 43) 699817697 52;

Institut für Staatsrecht und politische Wissenschaften, Abteilung für Öffentliches Unternehmensrecht, Johannes Kepler Universität Linz, Altenbergerstr. 69, A-4040 Linz, (00 43) 73224 68-84 57, Fax (00 43) 73224 68-89 01, E-Mail Nicolas.Raschauer@jku.at

486. Rasenack, Dr. Christian A.L., LL.M., Professor, Taunusstr. 8, 12309 Berlin, (0 30) 74525 43;

TU Berlin, Fakultät VIII, Institut für Volkswirtschaftslehre und Wirtschaftsrecht, Straße des 17. Juni 135, 10623 Berlin, (0 30) 31 42-58 74, Fax (0 30) 74525 43, E-Mail c.rasenack@ww.tu-berlin.de

487. Rauschning, Dr. Dr. h.c. Dietrich, o. Professor, Rodetal 1, 37120 Bovenden, (0 55 94) 931 74, Fax (0 55 94) 931 75; Institut für Völkerrecht, Universität Göttingen, Platz der Göttinger Sieben 5, 37073 Göttingen, (05 51) 3947 51, E-Mail drausch@gwdg.de

488. Reimer, Dr. Ekkehart, Professor, Institut für Finanz- und Steuerrecht, Lehrstuhl für Öffentliches Recht und Prinzipien des Europäischen und Internationalen Steuerrechts, Friedrich-Ebert-Anlage 6-10, 69117 Heidelberg, (0 62 21) 5474 67, Fax (0 62 21) 5477 91, E-Mail Reimer@uni-heidelberg.de 
489. Reimer, Dr. Franz, Professor, Ebelstr. 37, 35392 Gießen;

Justus-Liebig-Universität Gießen, Fachbereich 1 (Rechtswissenschaft), Hein-Heckroth-Str. 5, 35390 Gießen, E-Mail franz.reimer@recht.uni-giessen.de

490. Reinhardt, Dr. Michael, LL.M. (Cantab.), Professor, Auf dem Stumpelrott 9, 50999 Köln, (02 21) 3517 30;

Universität Trier, Fachbereich V, 54286 Trier, (06 51) 2 01-25 78, Fax (06 51) 20125 80, E-Mail reinharm@uni-trier.de

491. Remmert, Dr. Barbara, Universitätsprofessorin, Bei der Fruchtschranne 4, 72070 Tübingen; Eberhard Karls Universität Tübingen, Lehrstuhl für Öffentliches Recht, Geschwister-Scholl-Platz, 72074 Tübingen, E-Mail remmert@jura.uni-tuebingen.de

492. Rengeling, Dr. Hans-Werner, Universitätsprofessor, Langeworth 143, 48159 Münster, (02 51) 2120 38, Fax (02 51) 2120 44;

European Legal Studies Institute, Universität Osnabrück, Martinistr. 10, 49069 Osnabrück, (05 41) 9 69-45 05 oder -45 04, Fax (05 41) 9 69-62 82, E-Mail H.-W.Rengeling@t-online.de

493. Rensmann, Dr. Thilo, LL:M. (University of Virginia), Universitätsprofessor, Sedanstr. 12, 53173 Bonn, (0228) 2144 12;

Technische Universität Dresden, Juristische Fakultät, Lehrstuhl für Völkerrecht, Europarecht und Öffentliches Recht, 01062 Dresden, (0351) 463373 66, Fax (0351) 463374 65, E-Mail rensmann@jura.tu-dresden.de 
494. Ress, Dr. iur. Dr. rer. pol. Dr. iur. h.c. mult., Georg, em. Universitätsprofessor an der Universität der Saarlandes, Professor an der International University Bremen, Richter am EGMR a.D., Am Botanischen Garten 26/6, 66123 Saarbrücken, (06 81) 3023055 oder 3725 45, Fax (06 81) 3725 45, E-Mail ress@mx.uni-saarland.de

495. Rhinow, Dr. René, o. Professor, em. Ordinarius für öffentliches Recht an der Universität Basel, Leisenbergstr. 26, CH-4410 Liestal, (00 41) 6191199 35, E-Mail rene.rhinow@gmail.com

496. Richter, Dr. Dagmar, Privatdozentin, Birkenweg 90, 24211 Preetz;

Walther-Schücking-Institut für Internationales Recht an der CAU Kiel, Westring 400, 24098 Kiel, E-Mail drichter@internat-recht.uni-kiel.de

497. Riedel, Dr. Eibe H., Universitätsprofessor, Haagwiesenweg 19, 67434 Neustadt, (0 63 21) 848 19;

Lehrstuhl für Deutsches und Ausländisches Öffentliches Recht, Völkerrecht und Europarecht, Universität Mannheim, Schloß/Westflügel, 68131 Mannheim, (06 21) 1 81-14 17 oder 1418 oder 14 20-22, Fax (06 21) 1 81-14 19, E-Mail riedel@uni-mannheim.de

498. Rill, Dr. Heinz Peter, em. o. Univ.-Prof., Peter-Jordan-Str. 145, A-1180 Wien, (00 43-1) 4 79-86 74;

Forschungsinstitut für Europafragen, Wirtschaftsuniversität Wien, Althanstraße 39-45, A-1090 Wien, (00 43-1) 31336 46-65 oder -66

499. Rinken, Dr. Alfred, Universitätsprofessor, Präsident des Staatsgerichtshofs der Freien Hansestadt Bremen, Treseburger Str. 37, 28205 Bremen, (04 21) 4407 62, E-Mail rinken@uni-bremen.de 
500. Rixen, Dr. Stephan, Universitätsprofessor, Universität Bayreuth,

Rechts- und Wirtschaftswissenschaftliche Fakultät, Lehrstuhl für Öffentliches Recht, Sozialwirtschaftsund Gesundheitsrecht, Universitätsstraße 30, 95447 Bayreuth, (09 21) 55-6010, Fax (09 21) 55-6012, E-Mail stephan.rixen@uni-bayreuth.de

501. Robbers, Dr. Gerhard, Universitätsprofessor, Dagobertstr. 17, 54292 Trier, (06 51) 537 10;

Universität Trier, Postfach 38 25, 54286 Trier, (06 51) 2 01-25 42, Fax (06 51) 2 01-39 05, E-Mail Robbers@uni-trier.de

502. Röben, Dr. Volker, LL.M., Professor, School of Law Swansea University, Singleton Park, Swansea SA2 8PP, Wales, UK, (00 44) 17926027 23, Fax (00 44) 17922958 55, E-Mail V.Roeben@swansea.ac.uk

503. Rodi, Dr. Michael, M.A., Universitätsprofessor, Richardstr. 82, 12043 Berlin;

Ernst-Moritz-Arndt-Universität Greifswald, Lehrstuhl für Öffentliches Recht, Finanz- und Steuerrecht, 17487 Greifswald, (0 38 34) 8621 00, E-Mail mrodi@uni-greifswald.de

504. Röger, Dr. Ralf, Professor, Fachhochschule des Bundes für öffentliche Verwaltung, Fachbereich Bundespolizei, Ratzeburger Landstraße 4, 23562 Lübeck, (04 51) 2 03-17 36, Fax (04 51) 2 03-17 09, E-Mail roeger@roeger.info

505. Röhl, Dr. Hans Christian, Professor, Mainaustraße 207a, 78464 Konstanz, (0 75 31) 80714 46;

Universität Konstanz, Lehrstuhl für Staats- und Verwaltungsrecht, Europarecht und Rechtsvergleichung, Fach D 115, Universitätsstr. 10, 78457 Konstanz, (0 75 31) 88-23 13, Fax (0 75 31) 88-25 63, E-Mail hans.christian.roehl@uni-konstanz.de 
506. Ronellenfitsch, Dr. Michael, o. Professor, Augusta-Anlage 15, 68165 Mannheim, (06 21) 4123 34;

Universität Tübingen, Juristische Fakultät, Geschwister-Scholl-Platz, 72074 Tübingen, (0 70 71) 29721 09, Fax (0 70 71) 29749 05, E-Mail ronellenfitsch@jura.uni-tuebingen.de

507. Rossen-Stadtfeld, Dr. Helge, Professor, Marklandstraße 17, 81549 München, (0 89) 744279 29;

Universität der Bundeswehr München, Fakultät für Wirtschafts- und Organisationswissenschaften, Werner-Heisenberg-Weg 39, 85577 Neubiberg, (0 89) 60 04-46 04, Fax (0 89) 60 04-37 00, E-Mail helge.rossen-stadtfeld@unibw.de

508. Rossi, Dr. Matthias, Professor, Universität Augsburg, Juristische Fakultät, Lehrstuhl für Staats- und Verwaltungsrecht, Europarecht sowie Gesetzgebungslehre, Universitätsstr. 2, 86135 Augsburg, (08 21) 598-45 45, Sekr. -45 46, Fax (08 21) 598-45 47, E-Mail matthias.rossi@jura.uni-augsburg.de

509. Roth, Dr. Wolfgang, LL.M. (Michigan), apl. Prof., An der Elisabethkirche 48, 53113 Bonn, (02 28) 91252 73;

RAe Redeker Sellner Dahs \& Widmaier, Mozartstraße 4-10, 53115 Bonn, (02 28) 726 25-5 42, E-Mail roth@redeker.de

510. Rozek, Dr. Jochen, Universitätsprofessor, Pfaffendorfer Str. 1, 04105 Leipzig, (03 41) 22559 32;

Lehrstuhl für Staats- und Verwaltungsrecht, Verfassungsgeschichte und Staatskirchenrecht, Universität Leipzig, Burgstr. 27, 04109 Leipzig, (03 41) 97 35-1 71, Sekr. -1 70, Fax (03 41) 97 35-1 79, E-Mail rozek@uni-leipzig.de 
511. Ruch, Dr. Alexander, o. Professor em., ETH Zürich

Gartenstr. 85, CH-4052 Basel, (00 41) 6127236 22,

E-Mail ruch@recht.gess.ethz.ch

512. Rudolf, Dr. Walter, o. Professor, Rubensallee 55a, 55127 Mainz, (0 61 31) 719 42, E-Mail inge.rudolf@t-online.de;

FB Rechts- und Wirtschaftswissenschaften, Universität Mainz, 55099 Mainz, (0 61 31) 39-22 073, Sekretariat (0 61 31) 39-23 041

513. Ruffert, Dr. Matthias, Professor, Naumannstraße 12, 07743 Jena, (0 36 41) 2072 63;

Rechtswissenschaftliche Fakultät, Universität Jena,

Carl-Zeiss-Straße 3, 07743 Jena, (0 36 41) 9422 01, Fax (0 36 41) 9422 02, E-Mail m.ruffert@recht.uni-jena.de

514. Rüfner, Dr. Wolfgang, Professor, Hagebuttenstr. 26, 53340 Meckenheim, (0 22 25) 71 07, E-Mail Ruefner@t-online.de; zugehörig Universität zu Köln

515. Rühl, Dr. Ulli F. H., Professor, Hermann-Allmers-Str. 34, 28209 Bremen, (04 21) 34674 84;

Universität Bremen, Fachbereich 6 Rechtswissenschaft, Universitätsallee, GW 1, Postfach 3304 40, 28334 Bremen, (04 21) 2 18-46 06, Sekretariat: (04 21) 2 18-21 27, E-Mail uruehl@uni-bremen.de

516. Ruland, Dr. Franz, Professor, Geschäftsführer des Verbandes Deutscher Rentenversicherungsträger a.D., Honorarprofessor an der Johann-Wolfgang-Goethe Universität Frankfurt, Strasslacher Straße 1B, 81479 München, (0 89) 727797 92, Fax (0 89) 749094 82, E-Mail Ruland.Franz@t-online.de 
517. Rupp, Dr. Hans Heinrich, o. Professor, Am Marienpfad 29, 55128 Mainz, (0 61 31) 34588

518. Ruthig, Dr. Josef, Universitätsprofessor, Dreiweidenstr. 6, 65195 Wiesbaden; Johannes-Gutenberg-Universität Mainz, Fachbereich Rechts- und Wirtschaftswissenschaften, Lehrstuhl für Öffentliches Recht, Europarecht und Rechtsvergleichung, 55099 Mainz, (06131) 3920 964, Fax (06131) 3924 059, E-Mail Ruthig@uni-mainz.de

519. Rütsche, Dr. Bernhard, Professor, Jubiläumsstr. 87, CH-3005 Bern, (00 41) 313111584 , E-Mail bernhard.ruetsche@bluewin.ch; Universität Zürich, Rechtswissenschaftliches Institut, Treichlerstr. 10, CH-8032 Zürich, (00 41) 4463461 03, Fax (00 41) 446341589 , E-Mail bernhard.ruetsche@unilu.ch

520. Rux, Dr. Johannes, Privatdozent, Sophienstr. 32, 76133 Karlsruhe, (07 21) 38312 47, Fax (07 21) 38312 48, E-Mail rux@staatsrecht.info

521. Sachs, Dr. Michael, Universitätsprofessor, Dattenfelder Str. 7, 51109 Köln, (02 21) 8446 57, Fax (02 21) 8406 70;

Universität zu Köln, Lehrstuhl für Staats- und Verwaltungsrecht, Albertus-Magnus-Platz,Bauteil V, 2. OG, 50923 Köln, (02 21) 4 70-58 03, Fax (02 21) 4 70-51 35, E-Mail Sachs@uni-koeln.de

522. Sacksofsky, Dr. Ute, Professorin,

Bundenweg 16, 60320 Frankfurt am Main, (0 69) 956220 51, Fax (0 69) 956220 52;

Goethe-Universität, Fachbereich Rechtswissenschaft, Institut für öffentliches Recht, Grüneburgplatz 1, 60323 Frankfurt am Main, (0 69) 798342 85, Fax (0 69) 79834513 E-Mail Sacksofsky@jur.uni-frankfurt.de 
523. Salzwedel, Dr. Jürgen, o. Professor, Siebengebirgsstr. 86, 53229 Bonn, (02 28) 481710

524. Sarcevic, Dr. Edin, apl. Professor, Thomasiusstr. 15, 04009 Leipzig, (03 41) 60173 93;

Juristenfakultät Leipzig, Postfach 100920 , (03 41) 97352 10, Fax (03 41) 97352 18, E-Mail edin@rz.uni-leipzig.de

525. Saurer, Dr. Johannes, LL.M. (Yale), Privatdozent, Albtalstr. 9, 76137 Karlsruhe, (0721) 15640 557,

Universität Bayreuth, Rechtswissenschaftliche Fakultät, Lehrstuhl Öffentliches Recht IV, 95447 Bayreuth, (0921) 55 2945, E-Mail johannes.saurer@uni-bayreuth.de

526. Saxer, Dr. Urs, Titularprofessor, LL.M., Kantstrasse 15, CH-8044 Zürich, (00 41-44) 42240 42;

Steinbrüchel Hüssy Rechtsanwälte, Grossmünsterplatz 8, CH-8001 Zürich, (00 41-44) 26940 00, Fax (00 41-44) 26940 01, E-Mail Saxer@steinlex.ch

527. Schachtschneider, Dr. Karl Albrecht, o. Professor, Kaschachtschneider@web.de

528. Schambeck, Dr. Dr. h.c. mult. Herbert, o. Universitätsprofessor, Hofzeile 21, A-1190 Wien, (00 43-1) 3634 94;

Universität Linz, (00 43) 7322468424

529. Schefer, Dr. Markus, Professor,

Gartenstadt 18, CH-4142 Münchenstein/BL, (00 41) 6141136 28;

Universität Basel Juristische Fakultät, Lehrstuhl für Staatsund Verwaltungsrecht, Maiengasse 51, CH-4056 Basel, (00 41) 6126725 13, E-Mail markus.schefer@unibas.ch 
530. Schefold, Dr. Dian, Universitätsprofessor, Mathildenstraße 93, 28203 Bremen, (04 21) 725 76;

FB Rechtswissenschaft der Universität Bremen, Universitätsallee, GW 1, Postfach 3304 40, 28334 Bremen, (04 21) 2 18-6 60 07, Fax (04 21) 2 18-6 60 30, E-Mail schefold@uni-bremen.de

531. Schenke, Dr. Ralf P., o. Professor, Hofstattstr. 3, 97209 Veitshöchheim, (09 31) 30171131 ;

Julius-Maximilians-Universität Würzburg, Lehrstuhl für Öffentliches Recht, Deutsches, Europäisches und Internationales Steuerrecht, Domerschulstr. 16, 97070 Würzburg, (09 31) 31-8 23 60, Fax (09 31) 31-8 60 70, E-Mail schenke@jura.uni-wuerzburg.de

532. Schenke, Dr. Wolf-Rüdiger, o. Professor, Beim Hochwald 30, 68305 Mannheim, (06 21) 7442 00;

Universität Mannheim, 68131 Mannheim, (06 21) 18114 10, E-Mail Schenke@jura.uni-mannheim.de

533. Scherer, Dr. Joachim, LL.M., apl. Professor, Privatweg 9, 64342 Seeheim-Jugenheim, (0 62 57) 9037 39;

RAe Baker \& McKenzie, Bethmannstr. 50-54, 60311 Frankfurt am Main, (0 69) 299081 89, Fax (0 69) 299081 08, E-Mail Joachim.Scherer@Bakernet.com

534. Scherzberg, Dr. Arno, Professor, Wartburgstr. 34, 99094 Erfurt, (0361) 73747 61;

Universität Erfurt, Staatswissenschaftliche Fakultät, Postfach 900 221, 99105 Erfurt; (03 61) 7 37-47 61, (03 61) 7 37-47 60 (Sekr.), Fax (03 61) 7 37-47 09, E-Mail Arno.Scherzberg@uni-erfurt.de 
535. Scheuing, Dr. Dieter H., o. Professor, Finkenstr. 17, 97204 Höchberg, (09 31) 483 31, Fax (09 31) 4081 98;

Universität Würzburg, 97070 Würzburg, E-Mail Scheuing@jura.uni-wuerzburg.de

536. Schiedermair, Dr. Hartmut, o. Professor, Wittelsbacher Str. 7, 53173 Bonn-Bad Godesberg;

Institut für Völkerrecht und ausländisches öffentliches Recht, Universität Köln,

Gottfried-Keller-Str. 2, 50931 Köln, (02 21) 4702364

537. Schilling, Dr. Theodor, apl. Professor,

Le Mas des Roses, Fontcaudette,

F-84220 Gordes;

Humboldt-Universität zu Berlin, 10117 Berlin;

Gerichtshof der EG, L-2925 Luxemburg,

(0 03 52) 43 03-34 13,

E-Mail thsch@web.de

538. Schindler, Dr. Benjamin, MJur (Oxford), o. Professor,

Ober Bendlehn 32, CH_9042 Speicher;

Universität St. Gallen, Law School,

Tigerbergstraße 21, CH-9000 St. Gallen,

+41 (0)7122421 63, Fax +41 (0)712242162,

E-Mail benjamin.schindler@unisg.ch

539. Schindler, Dr. Dr. h.c. Dietrich, Professor,

Lenzenwiesstr. 8, CH-8702 Zollikon;

Universität Zürich,

(00 41-1) 3 91-71 18 oder -41 40,

Fax (00 41-1) 3 91-71 18

540. Schlacke, Dr. Sabine, Professorin,

Querstr. 9, 18107 Elmenhorst, (03 81) 51060 82;

Universität Bremen, Professur für Öffentliches Recht mit dem Schwerpunkt deutsches, europäisches und internationales Umweltrecht, Verwaltungsrecht Universitätsallee, 28353 Bremen, (04 21) 2 18-72 49, Fax (04 21) 2 18-74 90, E-Mail schlacke@uni-bremen.de 
541. Schlette, Dr. Volker, Professor, Hirberg 4, 37170 Uslar, (0 55 73) 9998 68;

Universität Göttingen, Juristisches Seminar, Platz der Göttinger Sieben 6, 37073 Göttingen, (05 51) 3944 13, Fax (05 51) 397414

542. Schlieffen, Dr. Katharina Gräfin von, Universitätsprofessorin, FernUniversität Hagen, Fachbereich Rechtswissenschaft, Universitätsstr. 21, 58084 Hagen, (0 23 31) 9 87-28 78, Fax (0 23 31) 9 87-3 95, E-Mail LG.vonSchlieffen@fernuni-hagen.de

543. Schliesky, Dr. Utz, apl. Professor, Direktor des Schleswig-Holsteinischen Landtages, Goosdiek 22, 24229 Dänischenhagen;

Schleswig-Holsteinischer Landtag, Düsternbrooker Weg 70, 24105 Kiel (04 31) 9 88-10 10;

Lorenz-von-Stein-Institut für Verwaltungswissenschaften an der Christian-Albrechts-Universität zu Kiel, Olshausenstr. 75, 24098 Kiel, E-Mail Utz.Schliesky@landtag.ltsh.de

544. Schlink, Dr. Bernhard, Professor, Viktoria-Luise-Platz 4, 10777 Berlin; Institut für Öffentliches Recht und Völkerrecht, Humboldt-Universität zu Berlin, Unter den Linden 6, 10099 Berlin, (0 30) 20 93-34 54 oder -34 72, Fax (0 30) 20 93-34 52, E-Mail Schlink@rewi.hu-berlin.de

545. Schmahl, Dr. Stefanie, LL.M., Professorin, Wittelsbacherstraße 10 A, 10707 Berlin; Lehrstuhl für deutsches und ausländisches öffentliches Recht, Völkerrecht und Europarecht, Universität Würzburg, Domerschulstr. 16, 97070 Würzburg, (09 31) 31-8 23 24, Fax (09 31) 31-27 92, E-Mail schmahl@jura.uni-wuerzburg.de 
546. Schmalenbach, Dr. Kirsten, Professorin, Richard Wagner Gasse 13, A-8010 Graz;

Institut für Völkerrecht,

Paris Lodron Universität Salzburg,

Churfürststraße 1, A-5020 Salzburg,

(00 43) 66280 44-36 51, Fax (00 43) 66280441 35,

E-Mail kirsten.schmalenbach@sbg.ac.at

547. Schmehl, Dr. Arndt, Universitätsprofessor,

Professur für Öffentliches Recht, Finanz- und Steuerrecht,

Universität Hamburg,

Schlüterstr 28 (Rechtshaus), 20146 Hamburg,

(0 40) 42838 -30 26 (Sekr.) und -30 25 (direkt),

Fax (0 40) 42838 -30 28,

E-Mail arndt.schmehl@jura.uni-hamburg.de

548. Schmid, Dr. Gerhard, Professor,

Hochwaldstr. 24, CH-4059 Basel,

(00 41) 6133184 25;

c/o Wenger Plattner, Aeschenvorstadt 55,

CH-4010 Basel,

(00 41) 612 79-70 00, Fax (00 41) 612 79-70 01,

E-Mail Gerhard.Schmid@wenger-plattner.ch

549. Schmid, Dr. Viola, LL.M., Universitätsprofessorin,

Kirchenweg 3, 91126 Schwabach,

(0 91 22) 773 82, Fax (0 91 22) 623 45;

Institut für Öffentliches Recht, Technische Universität Darmstadt,

Hochschulstr. 1, 64289 Darmstadt,

(0 61 51) 1664 64, Fax (0 61 51) 1639 84,

E-Mailschmid@jus.tu-darmstadt.de

550. Schmidt, Dr. Reiner, o. Professor,

Bachwiesenstr. 5, 86459 Gessertshausen,

(0 82 38) 41 11, Fax (0 82 38) 609 01,

E-Mail Rein.Schmidt@t-online.de;

Lehrstuhl für Öffentliches Recht, Wirtschaftsverwaltungsrecht und Umweltrecht, Universität Augsburg, Universitätsstr. 24, 86159 Augsburg, (08 21) 5 98-45 26 
551. Schmidt, Dr. Thorsten Ingo, Universitätsprofessor, Köhlerstr. 31, 12205 Berlin, (01 63) 135 54 87;

Professur für Öffentliches Recht, insbesondere Staatsrecht, Verwaltungs- und Kommunalrecht, Universität Potsdam,

August-Bebel-Str. 89, 14482 Potsdam

(03 31) 9 77-32 84,

E-Mail thorsten.ingo.schmidt@uni-potsdam.de

552. Schmidt, Dr. Walter, Universitätsprofessor,

Brüder-Knauß-Str. 86, 64285 Darmstadt, (0 61 51) 647 10;

Universität Frankfurt, 60054 Frankfurt am Main, (0 69) 79822189

553. Schmidt am Busch, Dr. Birgit, LL.M. (Iowa), Privatdozentin, Schmellerstr. 28, 80337 München, (0 89) 7257420 , E-Mail BSaB@aol.com;

Ludwig-Maximilians-Universität München, Ludwigstr. 28, Rgb., 80539 München, (0 89) 21 80-20 82,

E-Mail Schmidt-am-Busch@jura.uni-muenchen.de

554. Schmidt-Aßmann, Dr. Dres. h.c. Eberhard, o. Professor, Höhenstr. 30, 69118 Heidelberg, (0 62 21) 8008 03;

Universität Heidelberg, 69117 Heidelberg, (0 62 21) 5478 07, E-Mail schmidt-assmann@uni-hd.de

555. Schmidt-De Caluwe, Reimund, Universitätsprofessor, Unterer Hardthof 17 B, 35398 Gießen, (06 41) 345 66, Fax (06 41) 96099 66; Juristische Fakultät der Martin-Luther-Universität Halle-Wittenberg, Universitätsplatz 3-5, 06099 Halle (Saale), (03 45) 55-2 3138 oder -39, E-Mail Schmidtdc@jura.uni-halle.de 
556. Schmidt-Jortzig, Dr. Edzard, o. Professor, Graf-Spee-Straße 18a, 24105 Kiel, (04 31) 89501 95, Fax (04 31) 8034 71, Christian-Albrechts-Universität zu Kiel, Lehrstuhl für Öffentliches Recht, Leibnizstr.6, 24118 Kiel, (04 31) 8 80-35 45, E-Mail office.sj@law.uni-kiel.de

557. Schmidt-Preuß, Dr. Matthias, o. Professor, E.-T.-A.-Hoffmann-Straße 12, 53113 Bonn, (02 28) 6780 91;

Universität Bonn, Rechts- und Staatswissenschaftliche Fakultät, Adenauerallee 24-42, 53113 Bonn, (02 28) 7365 02, Fax (02 28) 7365 07, E-Mail schmidt-preuss@jura.uni-bonn.de

558. Schmidt-Radefeldt, Dr. Roman, Privatdozent, Schleiermacherstr.5, 68165 Mannheim, (06 21) 82075 02, E-Mail romansr69@yahoo.de;

Fachhochschule des Bundes für öffentliche Verwaltung, Fachbereich Bundeswehrverwaltung, Seckenheimer Landstr.30, 68163 Mannheim, (0621) 4295460 , E-Mail romanschmidtradefeldt@bundeswehr.org

559. Schmitt Glaeser, Dr. Alexander, LL.M. (Yale), Privatdozent, Kaulbachstraße 64, 80539 München, (0 89) 385479 31, E-Mail a.schmitt-glaeser@aya.yale.edu

560. Schmitt Glaeser, Dr. Dr. h. c. Walter, o. Professor, Rübezahlweg 9 A, 95447 Bayreuth, (09 21) 320 70, Fax (09 21) 7563866

561. Schmitt-Kammler, Dr. Arnulf, Universitätsprofessor, Katzenberg 6, 96049 Bamberg; Universität zu Köln, Rechtswissenschaftliche Fakultät, Albertus-Magnus-Platz, 50923 Köln, (02 21) 4 70-40 66 oder -40 67, E-Mail schmitt-kammler@gmx.de 
562. Schmitz, Dr. Thomas, Professor, (vormals Universität Lettlands, Riga),

Mittelstr. 1, 31535 Neustadt am Rübenberge, (01525) 48795 26,

E-Mail tschmit1@gwdg.de

563. Schnapp, Dr. Friedrich E., o. Professor, Efeuweg 22, 44869 Bochum, (0 23 27) 742 13; Universität Bochum, 44780 Bochum, (02 34) 32-2 22 39, Fax (02 34) 32-1 42 71, E-Mail friedrich.e.schnapp@rub.de

564. Schneider, Dr. Dr. h.c. Hans-Peter, em. Professor, Deutsches Institut für Föderalismusforschung,. Drosselweg 4, 30559 Hannover, (0511) 5110 50, Fax (0511) 5445 492; Juristische Fakultät, Leibniz-Universität Hannover, (0511) 7 62-8185

E-Mail hps@dif.uni-hannover.de

565. Schneider, Dr. Jens-Peter, Professor, Lorettostr. 36, 79100 Freiburg, (0761) 29086300 ;

Albert-Ludwigs-Universität Freiburg, Rechtswissenschaftliche Fakultät, 79085 Freiburg, (0761) 203-97731; Fax (0761) 203-97542

E-Mail jp.schneider@jura.uni-freiburg.de

566. Schöbener, Dr. Burkhard, Professor, Am Glösberg 27, 97342 Obernbreit, (0 93 32) 5000 04;

Professur für Öffentliches Recht, Völkerrecht und Europarecht, Universität zu Köln, Gottfried-Keller-Straße 2, 50931 Köln, (02 21) 4 70-38 34 oder -38 75, E-Mail burkhard.schoebener@uni-koeln.de 
567. Schönberger, Dr. Christoph, Professor, Wintererstr. 7, 79104 Freiburg i. Br., (07 61) 31968 72;

Universität Konstanz, Fachbereich Rechtswissenschaft, Postfach D 110, Universitätsstr. 10, 78457 Konstanz, (0 75 31) 8830 04, Fax (0 75 31) 8840 08, E-Mail Christoph.Schoenberger@uni-konstanz.de

568. Schoch, Dr. Friedrich, o. Professor, Kastelbergstr. 19, 79189 Bad Krozingen, (0 76 33) 9481 04, Fax (0 76 33) 9481 05;

Institut für Öffentliches Recht IV, Universität Freiburg, Postfach, 79085 Freiburg, (07 61) 2 03-22 57 oder -22 58, Fax (07 61) 2 03-22 97, E-Mail oerecht4@jura.uni-freiburg.de

569. Scholler, Dr. Dr. h. c. Heinrich, Professor, Zwengauerweg 5, 81479 München, (0 89) 796424 (privat), (0 89) 332014 (Büro), Fax (0 89) 7900216

570. Scholz, Dr. Rupert, o. Professor, Königsallee 71 a, 14193 Berlin;

Universität München, Institut für Politik und Öffentliches Recht, Ludwigstr. 28/RG, 80539 München, (0 89) 21 80-21 13, E-Mail rupert.scholz@jura.uni-muenchen.de

571. Schorkopf, Dr. Frank, Professor, Schillerstr. 49, 37083 Göttingen, E-Mail schorkopf@web.de;

Georg-August-Universität Göttingen, Juristische Fakultät, Institut für Völkerrecht und Europarecht, Platz der Göttinger Sieben 5, 37073 Göttingen, (05 51) 39-46 10, Fax (05 51) 39-2 21 96, E-Mail Frank.Schorkopf@jura.uni-goettingen.de

572. Schott, Dr. Markus, Privatdozent

Rütistr. 38, CH-8032 Zürich

(0041) 443631444

Bär \& Karrer AG, Brandschenkestr. 90, CH-8027 Zürich, (0041) 5826150 00, Fax (0041) 582635477

E-Mail markus.schott@baerkarrer.ch 
573. Schröder, Dr. Meinhard, o. Professor, Zum Wingert 2, 54318 Mertesdorf, (06 51) 578 87;

Universität Trier, 54286 Trier, (06 51) 2012586 ,

E-Mailschroedm@uni-trier.de

574. Schröder, Dr. Rainer Johannes, Privatdozent, Wormser Str. 65, 01309 Dresden, (03 51) 65697 00;

Technische Universität Dresden, Juristische Fakultät, Bergstr. 53, 01069 Dresden, (03 51) 46337365 , E-Mail rschroed@jura.tu-dresden.de

575. Schroeder, Dr. Werner, LL.M., Professor, Universität Innsbruck, Institut für Völkerrecht, Europarecht und Internationale Beziehungen, Innrain 52, A-6020 Innsbruck, (00 43) 5 12-5 07-83 20, Fax (00 43) 5 12-5 07-26 51, E-Mail Werner.Schroeder@uibk.ac.at

576. Schuler-Harms, Dr. Margarete, Professorin, Heidkoppel 19, 22145 Hamburg, (0 40) 67860 61, Fax (0 40) 67883 73;

Helmut-Schmidt-Universität, Universität der Bundeswehr Institut für Öffentliches Recht, Holstenhofweg 85, 22043 Hamburg, (0 40) 65 41-27 82, Fax (0 40) 65 41-20 87, E-Mail Schuler-Harms@hsu-hh.de

577. Schulev-Steindl, Dr. MMag. Eva, LL.M. (London), a.o. Univ. Prof., Auhofstraße 158/20, A-1130 Wien; Institut für Staats- und Verwaltungsrecht, Universität Wien, Schottenbastei 10-16, A-1010 Wien, (00 43-1) 42 77-3 5453 oder -51, Fax (00 43-1) 42 77-3 54 59, E-Mail eva.schulev-steind1@univie.ac.at 
578. Schulte, Dr. Martin, Professor, Neuostra 15, 01219 Dresden, (03 51) 47225 50;

Lehrstuhl für Öffentliches Recht, Umwelt- und Technikrecht, Juristische Fakultät, TU Dresden, von-Gerber-Bau, Bergstr. 53, 01069 Dresden, (03 51) 46 33-73 62, Fax (03 51) 46 33-72 20, E-Mail schulte@jura.tu-dresden.de

579. Schulz, Dr. Wolfgang, Privatdozent, Bismarckstr. 4, 20259 Hamburg, (0 40) 4040 75; Hans-Bredow-Institut für Medienforschung, Heimhuder Str. 21, 20148 Hamburg, (0 40) 45021711 (Sekr.), -34 (Durchwahl), Fax (0 40) 450217 77, E-Mailw.schulz@hans-bredow-institut.de

580. Schulze-Fielitz, Dr. Helmuth, Professor, Klara-Löwe-Str. 5, 97082 Würzburg, (09 31) 78410 25, Fax (09 31) 78410 34, E-Mail L-Schulze-Fielitz@jura.uni-wuerzburg.de

581. Schuppert, Dr. Gunnar Folke, Professor, Kaiserdamm 28, 14057 Berlin, (0 30) 30612168 ;

Wissenschaftszentrum Berlin für Sozialforschung, Forschungsprofessur Neue Formen von Governance, Reichpietschufer 50, 10785 Berlin, (0 30) 254 91-5 46 oder -246, Fax (0 30) 254 91-542, E-Mail schuppert@wzb.eu

582. Schwabe, Dr. Jürgen, Professor, Erlenweg 1, 21614 Buxtehude, (0 41 61) 871 41, Fax (0 41 61) 7226 00; Universität Hamburg, Juristische Fakultät, Schlüterstraße 28, 20146 Hamburg, E-Mail Juergen_Schwabe@gmx.de

583. Schwartmann, Dr. Rolf, Professor, Brucknerstraße 18, 50931 Köln, (02 21) 40090 94;

Fachhochschule Köln, Fakultät für Wirtschaftswissenschaften, Claudiusstraße 1, 50678 Köln, (02 21) 82 75-34 46, Fax (02 21) 82 75-7 34 46, E-Mail rolf.schwartmann@fh-koeln.de 
584. Schwarz, Dr. Kyrill-A., Professor, Dönersberg 13, 91550 Dinkelsbühl, (01 77) 83107 68;

Universität Würzburg, Juristische Fakultät, Professor für Öffentliches Recht, Domerschulstr. 16, 97070 Würzburg, (0931) 318-2335

E-Mail kyrill-alexander.schwarz@uni-wuerzburg.de

585. Schwarze, Dr. Jürgen, Professor,

Universität Freiburg, Institut für Öffentliches Recht Abt. I, Platz der Alten Synagoge 1, 79098 Freiburg,

(07 61) 2 03-22 38, oder -22 51, Fax (07 61) 2 03-22 34,

E-Mail juergen.schwarze@jura.uni-freiburg.de

586. Schwarzer, Mag., Dr. Stephan, Universitätsdozent, Rodlergasse 7/10, A-1190 Wien, (00 43-1) 36917 46;

Bundeswirtschaftskammer, Wiedner Hauptstr. 63, A-1045 Wien, (00 43-1) $50105-4195$

587. Schweitzer, Dr. Michael, Professor, Göttweiger Str. 135, 94032 Passau, (08 51) 345 33;

Universität Passau, 94032 Passau, (08 51) 5 09-23 30, Fax (08 51) 5 09-23 32,

E-Mail Schweitzer@uni-passau.de

588. Schweizer, Dr. Rainer J., o. Professor, Kirchgasse 9, CH-9220 Bischofszell, (00 41) 71-2 2356 24; Universität St. Gallen, Tigerbergstr. 21, CH-9000 St. Gallen, Forschungsgemeinschaft für Rechtswissenschaften, (00 41) 71-2 2421 61, Fax (00 41) 71-2 2421 62, E-Mail Rainer.Schweizer@unisg.ch

589. Schwerdtfeger, Dr. Gunther, Universitätsprofessor, Hülsebrinkstr. 23, 30974 Wennigsen/Deister, (0 5103 ) 13 11;

Juristische Fakultät, Universität Hannover, Königsworther Platz 1, 30167 Hannover, (05 11) 7 62-81 69 
590. Seer, Dr. Roman, Universitätsprofessor, In den Birken $156 \mathrm{~d}, 42113$ Wuppertal, (02 02) 27215 34, Fax (02 02) 27215 35;

Ruhr-Universität Bochum, Lehrstuhl für Steuerrecht, Gebäude GC 8/137, Universitätsstr. 150, 44780 Bochum, (02 34) 32282 69, Fax (02 34) 32146 14, E-Mail LS.seer@jura.ruhr-uni-bochum.de

591. Seewald, Dr. Otfried, o. Professor, Schärdingerstraße 21 A, 94032 Passau, (08 51) 351 45, Fax (08 51) 351 45, E-Mail otfried_seewald@gmx.de; Universität Passau, Innstr. 40, Postfach 25 40, 94030 Passau, (08 51) 50 9-23 40 oder -41, Fax (08 51) 5 09-23 42, E-Mail otfried.seewald@uni-passau.de

592. Seidel, Dr. Gerd, Professor, Donizettistraße 102, 12623 Berlin, (0 30) 565975 56;

Humboldt Universität zu Berlin, Lehrstuhl für Öffentliches Recht, Völker- und Europarecht, Unter den Linden 6, 10099 Berlin, (0 30) 20 93-35 17/-12, Fax (0 30) 20 93-33 84, E-Mail gerd.seidel@rewi.hu-berlin.de

593. Seiler, Dr. Christian, Professor, Stauffenbergstr. 70/1, 72074 Tübingen, (0 70 71) 54977 80; Universität Tübingen, Lehrstuhl für Staatsund Verwaltungsrecht, Finanz- und Steuerrecht, Geschwister-Scholl-Platz, 72074 Tübingen, (0 70 71) 29729 43, E-Mail christian.seiler@jura.uni-tuebingen.de

594. Selmer, Dr. Peter, Professor, Akazienweg 9, 22587 Hamburg, (0 40) 8647 43; Universität Hamburg, 20146 Hamburg, (0 40) 428 38-45 74 oder -3026, Fax (0 40) 428 38-30 28, E-Mail peter.selmer@jura.uni-hamburg.de 
595. Shirvani, Dr. Foroud, Privatdozent, Grafinger Str. 91, 81671 München, (0 89) 490036 63;

Ludwig-Maximilians-Universität München, Ludwigstr. 28 (Rgb.), 80539 München, (0 89) 218028 83, Fax (0 89) 218031 99, E-Mail foroud.shirvani@jura.uni-muenchen.de

596. Sieckmann, Dr. Jan-Reinhard, Professor,

Sozial- und Wirtschaftswissenschaftliche Fakultät, Otto-Friedrich-Universität Bamberg,

Feldkirchenstr. 21, 96051 Bamberg, (09 51) 8 63-27 40, Fax (09 51) 8 63-57 40, E-Mail Jan.Sieckmann@sowi.uni-bamberg.de

597. Siedentopf, Dr. Dr. h.c. Heinrich, o. Professor, Langstr. 1, 76829 Landau, (0 63 41) 607 57;

Deutsche Hochschule für Verwaltungswissenschaften Speyer, Freiherr-vom-Stein-Str. 2-6, 67324 Speyer, E-Mail heinrich@siedentopf.net

598. Siegel, Dr. Thorsten, Privatdozent, Dr. Semmelweis-Str. 25, 67433 Neustadt an der Weinstraße, (0 63 21) 4828 51;

Deutsches Forschungsinstitut für öffentliche Verwaltung Speyer, Freiherr-vom-Stein-Str. 2, 67346 Speyer, (0 62 32) 6 54-3 79, Fax (0 62 32) 6 54-2 90, E-Mail siegel@foev-speyer.de

599. Siehr, Dr. Angelika, LL.M. (Yale), Privatdozentin, Zickzackredder 9, 24850 Schuby, (04621) 9488 88;

Universität Bielefeld, Rechtswissenschaftliche Fakultät, Postfach 100131, 33501 Bielefeld, (0521) 106-4430, (0521) 106-6893 (Sekretariat), E-Mail angelika.siehr@rewi.hu-berlin.de

600. Siekmann, Dr. Helmut, Professor, Johann Wolfgang Goethe-Universität, Professur für Geld-, Währungs- und Notenbankrecht, IMFS im House of Finance, Grüneburgplatz 1, 60323 Frankfurt am Main, (0 69) 7 98-3 4014 
601. Silagi, Dr. Dr. Michael, Privatdozent, Institut für Völkerrecht, Universität Göttingen, Platz der Göttinger Sieben 5, 37073 Göttingen, (05 51) 39-47 34

602. Skouris, Dr. Wassilios, Professor, Nikolaou Manou 18, GR-54643 Thessaloniki, (00 30-31) 8314 44;

Gerichtshof der Europäischen Gemeinschaften, Palais de la Cour de Justice, L-2925 Luxembourg, (l $\left.0 \begin{array}{lll}0 & 52\end{array}\right) 430322$ 09, Fax (0 03 52) 43032736

603. Smeddinck, Dr. Ulrich, Privatdozent, Sommerhuder Str. 35, 22769 Hamburg, (01 74) 24899 90;

Umweltbundesamt

Wörlitzer Platz 1, FB III, 06844 Dessau, (03 40) 21 03-20 77, E-Mail ulrich.smeddinck@uba.de Martin-Luther-Universität Halle-Wittenberg, Juristische und Wirtschaftswissenschaftliche Fakultät, Universitätsplatz 10a, 06108 Halle/Saale, E-Mail Ulrich.Smeddinck@jura.uni-halle.de

604. Sodan, Dr. Helge, Universitätsprofessor,

Fachbereich Rechtswissenschaft, Lehrstuhl für Staats- und Verwaltungsrecht, Öffentliches Wirtschaftsrecht, Sozialrecht, Freie Universität Berlin, Van't-Hoff-Str. 8, 14195 Berlin, (0 30) 8 38-5 3972 oder -7 39 73, Fax (0 30) 8 38-5 44 44; Präsident des Verfassungsgerichtshofes des Landes Berlin, Elßholzstr. 30-33, 10781 Berlin, (0 30) 90 15-26 50, Fax (0 30) 90 15-26 66, E-Mailsodan@zedat.fu-berlin.de

605. Söhn, Dr. Hartmut, o. Professor, Eppanerstr. 9, 94036 Passau, (08 51) 58520 , E-Mail hsoehndr@web.de; Universität Passau, Lehrstuhl für Staats- u. Verwaltungsrecht insbesondere Finanz- und Steuerrecht, 94032 Passau, (08 51) 5 09-23 50, Fax (08 51) 5 09-23 52 
606. Somek, Dr. Alexander, Professor, Görgengasse 23a/8, A-1190 Wien; University of Iowa, College of Law, Melrose and Byington Iowa City, Iowa USA 52242, (3 19) 33590 34, Fax (31 91) 335901 98, E-Mail alexander-somek@uiowa.edu

607. Sommermann, Dr. Karl-Peter, Universitätsprofessor, Lehrstuhl für Öffentliches Recht, Staatslehre und Rechtsvergleichung, Deutsche Hochschule für Verwaltungswissenschaften Speyer, Postfach 14 09, 67346 Speyer, (0 62 32) 6 54-3 44, Fax (0 62 32) 6 54-4 14, E-Mail Sommermann@dhv-speyer.de

608. Spannowsky, Dr. Willy, Universitätsprofessor, Auf dem Kleehügel 17, 67706 Krickenbach, (0 63 07) 9939 63, Fax (0 63 07) 9939 49;

Lehrstuhl für Öffentliches Recht, Postfach 30 49, 67653 Kaiserslautern, (06 31) 2 05-39 75, Fax (06 31) 2 05-39 77, E-Mail oerecht@rhrk.uni-kl.de

609. Nur TextSpiecker genannt Döhmann, Dr. Indra, LL.M.

(Georgetown Univ.), Professorin, Institut für Informations- und Wirtschaftsrecht Nur TextZentrum für Angewandte Rechtswissenschaft (ZAR) Nur TextKarlsruher Institut für Technologie (KIT)

Vincenz-Prießnitz-Str. 3, Geb. 07.08, 76131 Karlsruhe,

(07 21) 6084 -77 59, Fax (07 21) 6084 - 80 23, E-Mail spiecker@kit.edu

Nur Text www.zar.kit.edu

610. Spranger, Dr. Dr. Tade Matthias, Privatdozent, Eichhörnchenweg 10, 53125 Bonn;

c/o Institut für Wissenschaft und Ethik, Bonner Talweg 57, 53113 Bonn, (02 28) 336419 53, Fax (02 28) 336419 50, E-Mail spranger@iwe.uni-bonn.de

611. Staff, Dr. Ilse, Universitätsprofessorin, Am Forum 4, 65779 Kelkheim, (0 61 95) 33 08; Universität Frankfurt, 60054 Frankfurt am Main 
612. Starck, Dr. Christian, o. Professor, Schlegelweg 10, 37075 Göttingen, (05 51) 554 54, E-Mail C.Starck@jura.uni-goettingen.de

613. Steiger, Dr. Heinhard, Universitätsprofessor, Oberhof 16, 35440 Linden, (06 41) 232 52; Universität Gießen, 35394 Gießen, (06 41) 992 11-50 oder -51, Fax (06 41) 992 11-59

614. Stein, Dr. Katrin, Privatdozentin, Reinhold-Tiling-Weg 61, 49088 Osnabrück, (05 41) 91184 51; Universität Osnabrück, Institut für Kommunalrecht, Martinistr. 12, 49078 Osnabrück, (05 41) 9 69-61 69, E-Mail kstein@uos.de

615. Stein, Dr. Torsten, Universitätsprofessor, Ludolf-Krehl-Str. 1 b, 69120 Heidelberg, (0 62 21) 4804 38, Fax (0 62 21) 4804 38; Universität des Saarlandes, Europa-Institut, Am Stadtwald, 66041 Saarbrücken, (06 81) 3 02-45 67 oder -36 95, Fax (06 81) 3 02-48 79, E-Mail t.stein@mx.uni-saarland.de

616. Steinberg, Dr. Rudolf, Universitätsprofessor, Universitätspräsident a.D., Wingertstr. 2 A, 65719 Hofheim; Goethe-Universität Frankfurt am Main, Grüneburgplatz 1, 60323 Frankfurt, E-Mail Rudolf-Steinberg@t-online.de

617. Steinberger, Dr. Helmut, o. Professor, Saphirweg 13, 69181 Leimen, (062) 26990630 ;

MPI für ausländisches öffentliches Recht und Völkerrecht, Im Neuenheimer Feld 535, 69120 Heidelberg, (0 62 21) 4 82-2 61, Fax (0 62 21) 4 82-2 88; Juristisches Seminar, Universität Heidelberg, Friedrich-Ebert-Anlage 6-10, 69117 Heidelberg, (0 62 21) 54-74 54 oder -74 55, Fax (0 62 21) 54-77 44 E-Mail hsteinber@mpil.de 
618. Steiner, Dr. Udo, o. Professor, Richter des Bundesverfassungsgerichts a.D., Am Katzenbühl 5, 93055 Regensburg, (09 41) 7009 13, Fax (09 41) 7606 19, E-Mail udo.steiner@web.de; Universität Regensburg, 93040 Regensburg, (09 41) 9 43-42 84,

E-Mail udo.steiner@jura.uni-regensburg.de

619. Stelkens, Dr. Ulrich, Universitätsprofessor, Webergasse 3a, 67346 Speyer;

Deutsche Hochschule für Verwaltungswissenschaften Speyer, Freiherr-vom-Stein-Str. 2, 67346 Speyer, (0 62 32) 6 54-3 65, Fax (0 62 32) 6 54-2 45, E-Mail stelkens@dhv-speyer.de

620. Stelzer, Dr. Manfred, Universitätsprofessor, Anton-Wildgansgasse 12/4, A-2380 Perchtoldsdorf, (00 43) 66421256 18;

Universität Wien, Schottenbastei 10-16, A-1010 Wien, (00 43-1) 42 77-3 5431 oder -32, E-Mail Manfred.Stelzer@univie.ac.at

621. Stender-Vorwachs, Dr. Jutta, LL. M. (USA,UVA), apl. Professorin, Am Ortfelde 99A, 30916 Isernhagen N.B., (05 11) 72408 07, Fax (05 11) 72408 54, E-Mail jutta.stender-vorwachs@gmx.de; Leibniz Universität Hannover, Juristische Fakultät, Königsworter Platz 1, 30167 Hannover, (05 11) 7 62-82 50 oder -82 49, Fax (05 11) 7 62-82 52, E-Mail jutta.stender-vorwachs@jura.uni-hannover.de

622. Stern, Dr. Dr. h.c. mult. Klaus, o. Professor, Am Stockberger Busch 10, 51515 Kürten, (0 22 68) 61 67; Institut für Rundfunkrecht an der Universität zu Köln, Aachener Straße 197-199, 50931 Köln, (02 21) 94154 65, E-Mail klaus.stern@uni-koeln.de 
623. Stettner, Dr. Rupert, Professor,

Alpenstr. 11 a, 85221 Dachau,

(0 81 31) 2789 96, Fax (0 81 31) 2789 98;

Institut für Staatswissenschaften,

Universität der Bundeswehr München,

Werner-Heisenberg-Weg 39, 85579 Neubiberg,

(0 89) 60 04-38 64 oder -37 02 oder -20 43, Fax (0 89) 60 04-28 41,

E-Mail Rupert.Stettner@unibw-muenchen.de

624. Stober, Dr. Dr. h.c. mult. Rolf, Universitätsprofessor,

Prins-Claus-Str. 50, 48159 Münster,

(02 51) 16241 62, Fax (02 51) 1624163 ,

E-Mail rolf-stober@gmx.de;

Universität Hamburg,

E-Mail rolf.stober@mba.hamburg.de;

Deutsche Universität für Weiterbildung,

Pacelliallee 55, 14195 Berlin, (030) 2000306 253, Fax (030) 2000306 292,

E-Mail rolf.stober@duw-berlin.de

625. Stock, Dr. Martin, Professor,

Lina-Oetker-Str. 22, 33615 Bielefeld,

(05 21) 1219 95;

Fakultät für Rechtswissenschaft, Universität Bielefeld,

Postfach 1001 31, 33501 Bielefeld,

(05 21) 10643 90, Fax (05 21) 1061543 90,

E-Mail martin.stock@uni-bielefeld.de

626. Stöger, Dr. Karl, MJur, Universitätprofessor,

Höhenstr. 24-26, A-3400 Klosterneuburg,

(00 43) 0699103677 45;

Karl-Franzens-Universität Graz,

Institut für Österreichisches, Europäisches und Vergleichendes

Öffentliches Recht, Politikwissenschaft und Verwaltungslehre,

Universitätsstr. 15/C3, A-8010 Graz,

(0043) 0316380 3384,

E-Mail karl.stoeger@uni-graz.at 
627. Stoll, Dr. Peter-Tobias, Professor,

E-Mail ptstoll@web.de;

Institut für Völkerrecht, Abteilung für Internationales

Wirtschaftsrecht,

Universität Göttingen, Platz der Göttinger Sieben 5,

37073 Göttingen,

(05 51) 3946 61,

E-Mail pt.stoll@jur.uni-goettingen.de

628. Stolleis, Dr. Dr. h.c. mult. Michael, Universitätsprofessor,

Waldstr. 15, 61476 Kronberg,

(0 61 73) 65651 ;

Universität Frankfurt, MPI für europäische Rechtsgeschichte, Hausener Weg 120, 60489 Frankfurt am Main,

(0 69) 7 89 78-2 22, Fax (0 69) 789 78-1 69,

E-Mail Stolleis@mpier.uni-frankfurt.de

629. Stolzlechner, Dr. Harald, o. Universitätsprofessor,

Gneiser Straße 57, A-5020 Salzburg,

(00 43) 6 62-82 39 35;

Universität Salzburg,

(00 43) 6 62-80 4436 01,

E-Mail Harald.Stolzlechner@sbg.ac.at

630. Storr, Dr. Stefan, Universitätsprofessor,

Klosterwiesgasse 72, A-8010 Graz;

Institut für Österreichisches, Europäisches und Vergleichendes

Öffentliches Recht, Politikwissenschaft und Verwaltungslehre,

Karl-Franzens-Universität Graz,

Universitätsstraße 15 C 3, A-8010 Graz,

(00 43) 3163 80-66 95, Fax (00 43) 3163 80-94 50,

E-Mail stefan.storr@uni-graz.at

631. Streinz, Dr. Rudolf, o. Professor,

Waldsteinring 26, 95448 Bayreuth, (09 21) 947 30;

E-Mail rudolf.streinz@gmx.de

Ludwig-Maximilians-Universität München,

Lehrstuhl für Öffentliches Recht und Europarecht,

Professor-Huber-Platz 2, 80539 München,

(0 89) 21 80-33 35, Fax (0 89) 21 80-24 40,

E-Mail streinz.pers@jura.uni-muenchen.de 
632. Standard (Web)Stumpf, Dr. Dr. Christoph, Privatdozent, Raupach \& Wollert-Elmendorff Rechtsanwaltsgesellschaft $\mathrm{mbH}$ Hanse-Forum, Axel-Springer-Platz 3, 20355 Hamburg, (0 40) 3785 38-0, (0 40) 3785 38-11, E-Mail cstumpf@raupach.de

633. Suerbaum, Dr. Joachim, o. Professor, In der Uhlenflucht 3, 44795 Bochum, (02 34) 472626 ,

E-Mail Joachim.Suerbaum@t-online.de;

Universität Würzburg, Domerschulstraße 16, 97070 Würzburg, (09 31) 31-8 2897 oder 31-8 28 99, E-Mail Suerbaum@jura.uni-wuerzburg.de

634. Sydow, Dr. Gernot, M.A., apl. Prof., Schornstr. 13, 65553 Limburg, (0 64 31) 57095 20; Justitiar des Bistums Limburg, Bischöfliches Ordinariat, Roßmarkt 4, 65549 Limburg, (0 64 31) 2952 08, Fax (0 64 31) 2955 21, E-Mail G.Sydow@bistumlimburg.de, Gernot.Sydow@jura.uni-freiburg.de

635. Talmon, D. Phil. (Oxon.) Stefan, LL.M. (Cantab.), Privatdozent, Universität Tübingen, Geschwister-Scholl-Platz, 72074 Tübingen; St Anne's College, Woodstock Road, Oxford OX2 6HS, (00 44) 18652845 30, Fax (00 44) 18652748 99, E-Mail Stefan.Talmon@law.ox.ac.uk

636. Thieme, Dr. Werner, Professor, Berggartenstraße 14, 29223 Celle, (0 51 41) 373 69, Fax (0 51 41) 9313 73; Universität Hamburg, 20146 Hamburg, (0 40) $42838-4569$

637. Thiel, Dr. iur. Dr. rer. publ. Markus, Privatdozent, Gleueler Str. 114-116, 50935 Köln, (02 21) 408783 ;

Heinrich-Heine-Universität Düsseldorf, Juristische Fakultät, Universitätsstr. 1, 40225 Düsseldorf, (02 11) 81114 24, Fax (02 11) 8111455 , E-Mail markus.thiel@uni-duesseldorf.de 
638. Thienel, Dr. Rudolf, Universitätsprofessor, Vizepräsident des Verwaltungsgerichtshofes, Judenplatz 11, A-1010 Wien, (00 43-1) 531 11-2 45, Fax (00 43-1) 531 11-1 40, E-Mail rudolf.thienel@vwgh.gv.at

639. Thürer, Dr. Dr. h.c. Daniel, LL.M., o. Professor, Abeggweg 20, CH-8057 Zürich, (00 41) 13626547 oder -46, Fax (00 41)13 6265 46, E-Mail thuerer@swissonline.ch; Universität Zürich, Institut für Völkerrecht und Ausländisches Verfassungsrecht, Hirschgraben 40, CH-8001 Zürich, (00 41) 16 34-20 31 oder -2059 oder -2064, Fax (00 41) 16 34-49 92, E-Mail thuerer@ivr.unizh.ch

640. Thym, Dr. Daniel, LL.M. (London), Professor, Pallasstr. 22, 10781 Berlin, (01 51) 566069 37;

Universität Konstanz, Lehrstuhl für Öffentliches Recht, Europa- und Völkerrecht, Fach 116, Universitätsstr. 10, 78457 Konstanz, E-Mail daniel.thym@uni-konstanz.de

641. Tietje, Dr. Christian, Professor, Heinrich-Heine-Str. 8, 06114 Halle (Saale), (03 45) 5483912 oder $(03$ 45) 5248312 , Mobil (0175) 3736 134, Fax (03 45) 51740 48, Martin-Luther-Universität Halle-Wittenberg, Juristische Fakultät, Juridicum, Universitätsplatz 5, 06108 Halle (Saale), (03 45) 5 52-31 80, Fax (03 45) 5 52-72 01, E-Mail tietje@jura.uni-halle.de

642. Tomuschat, Dr. Christian, Professor, Odilostraße 25a, 13467 Berlin, (0 30) 405414 86, Fax (0 30) 405414 88, Humboldt-Universität zu Berlin, Institut für Völker- und Europarecht, Unter den Linden 6, 10099 Berlin, (0 30) 20 93-33 35 oder -3305 oder -3322 , Fax (0 30) 20 93-33 65, E-Mail christian.tomuschat@rewi.hu-berlin.de 
643. Trute, Dr. Hans-Heinrich, Universitätsprofessor, Wettinplatz 3, 01896 Pulsnitz, (03 5955 ) 453 01;

Universität Hamburg, Fakultät für Rechtswissenschaft, Schlüterstraße 28, 20146 Hamburg, (0 40) 428 38-57 21 oder -56 25, Fax (0 40) 428 38-27 00, E-Mail Hans-Heinrich.Trute@jura.uni-hamburg.de

644. Tschentscher, Dr. Axel, LL.M., Professor,

Lehrstuhl für Staatsrecht, Rechtsphilosophie und Verfassungsgeschichte, Universität Bern, Institut für öffentliches Recht, Schanzeneckstraße 1, CH-3001 Bern, (00 41) 31-6 318899 (direkt), (00 41) 31-6 313236 (Sekretariat), Fax (00 41) 31-6 3138 83, E-Mail axel.tschentscher@oefre.unibe.ch

645. Uebersax, Dr. Peter, Privatdozent, Chemin des Grands-Champs 19, $\mathrm{CH}-1033$ Cheseaux, (00 41) 2173129 41; Schweizerisches Bundesgericht, Av. du Tribunal-fédéderal 29, CH-1000 Lausanne 14, (00 41) 2131891 11, E-Mail peter.uebersax@bger.admin.ch

646. Uerpmann-Wittzack, Dr. Robert, Professor, Universität Regensburg, Juristische Fakultät, 93040 Regensburg, (09 41) 9 43-26 60 oder 26 59, Fax (09 41) 9 43-19 73, E-Mail Robert.Uerpmann@jura.uni-regensburg.de

647. Uhle, Dr. Arnd, Professor, Stiftungslehrstuhl für Öffentliches Recht, insbesondere für Staatsrecht und Staatswissenschaften, Technischen Universität Dresden, 01062 Dresden, (03 51) 463373 14, Fax (03 51) 463372 07, E-Mail uhle@jura.tu-dresden.de

648. Uhlmann, Dr. Felix, LL.M., Professor, Bruderholzallee, CH 4059 Basel;

Rämistrasse 74 / 33, CH 8001 Zürich, (00 41) 4463442 24, Fax (00 41) 4463443 68, E-Mail felix.uhlmann@rwi.unizh.ch 
649. Unruh, Dr. Peter, apl. Professor, Hakensoll 8a, 24226 Heikendorf; Nordelbisches Kirchenamt, Dänische Str. 21-35, 24103 Kiel, E-Mail punruh.nka@nordelbien.de

650. Vallender, Dr. Klaus A., Professor, Unterbach 4, CH-9043 Trogen, (00 41 71) 9427 69; Universität St. Gallen, Bodanstr. 4, CH-9000 St. Gallen, (00 41 71) 2242519

651. Vedder, Dr. Christoph, Professor, Sollner Str. 33, 81479 München, (0 89) 791003 83, Fax (0 89) 791003 84; Juristische Fakultät, Universität Augsburg, Postfach, 86135 Augsburg, (08 21) 5 98-45 70, Fax (08 21) 5 98-45 72, E-Mail christoph.vedder@jura.uni-augsburg.de

652. Vesting, Dr. Thomas, Universitätsprofessor, Konradstraße 2, 80801 München, (089) 388795 45, Fax (089) 38879547 Lehrstuhl für Öffentliches Recht, Recht und Theorie der Medien, Johann Wolfgang Goethe-Universität, Grüneburgplatz 1, RuW 04, 60629 Frankfurt am Main, (0 69) 79834 274, Fax (0 69) 798763 34273, E-Mail T.Vesting@jur.uni-frankfurt.de

653. Vitzthum, Dr. Dr. h.c. Wolfgang Graf, o. Professor, Im Rotbad 19, 72076 Tübingen, (0 70 71) 638 44, Fax (0 70 71) 9684 89; Universität Tübingen, Juristische Fakultät, Geschwister-Scholl-Platz, 72074 Tübingen, (0 70 71) 29752 66, Fax (0 70 71) 29750 39, E-Mail wolfgang-graf.vitzthum@uni-tuebingen.de 
654. Vöneky, Dr. Silja, Professorin, Am Schmelzofen 20, 79183 Waldkirch, (07681) 4925 239;

Albert-Ludwigs-Universität Freiburg, Institut für Öffentliches Recht, Abt. II Völkerrecht und Rechtsvergleichung, 79085 Freiburg im Breisgau, (0761) 203 2207, Fax (0761) 203 9193, E-Mail voelkerrecht@jura.uni-freiburg.de

655. Vogel, Dr. Stefan, Privatdozent, Dübendorfstr. 11c, CH-8117 Fällanden, (00 41) 433555229 , E-Mail stefan_vogel@bluewin.ch

656. Volkmann, Dr. Uwe, Professor, Am Bonifatiusbrunnen 231, 60438 Frankfurt am Main, (0 69) 518673 ;

Johannes-Gutenberg-Universität Mainz, FB Rechts- und Wirtschaftswissenschaften, Lehrstuhl für Rechtsphilosophie und Öffentliches Recht, 55099 Mainz,

(0 61 31) 39-2 34 53, Fax (0 61 31) 39-2 30 90, E-Mail Volkmann@uni-mainz.de

657. Voßkuhle, Dr. Andreas, Professor, Präsident des Bundesverfassungsgerichts, Schloßbezirk 3, 76131 Karlsruhe, (07 21) 91 01-3 13;

Albert-Ludwigs-Universität Freiburg, Institut für Staatswissenschaft und Rechtsphilosophie, Postfach, 79085 Freiburg i. Br., (07 61) 2 03-22 09, Fax (07 61) 2 03-91 93, E-Mail staatswissenschaft@jura.uni-freiburg.de

658. Waechter, Dr. Kay, Professor,

Ceciliengärten 12, 12159 Berlin;

FB Rechtswissenschaft, Universität Hannover, Königsworther Platz 1, 30167 Hannover, (05 11) 7 62-82 27, E-Mailwaechter@jura.uni-hannover.de 
659. Wahl, Dr. Rainer, o. Professor, Hagenmattenstr. 6, 79117 Freiburg, (07 61) 659 60;

Universität Freiburg, Institut für Öffentliches Recht V, Postfach, 79085 Freiburg, (07 61) 20389 61, Fax (07 61) 20322 93, E-Mail rainer.wahl@jura.uni-freiburg.de

660. Waldhoff, Dr. Christian, Professor, Lennéstraße 47, 53113 Bonn, (02 28) 28910 64;

Universität Bonn, Lehrstuhl für Öffentliches Recht, Kirchenrechtliches Institut, Adenauerallee 24-42, 53113 Bonn, (02 28) 73-91 25, Fax (02 28) 73-40 49, E-Mail waldhoff@uni-bonn.de

661. Waldmann, Dr. Bernhard, Professor, RA Alfons-Aebystrasse 29, CH-3186 Düdingen, (00 41) 2649357 05;

Universität Freiburg i. Üe.

Route d' Englisberg 7, CH-1763 Granges-Paccot, (00 41) 2630081 56, E-Mail bernhard.waldmann@unifr.ch

662. Wallerath, Dr. Maximilian, Universitätsprofessor, Gudenauer Weg 86, 53127 Bonn, (02 28) 2832 02, E-Mail max.wallerath@web.de; Universität Greifswald, Rechts- und Staatswissenschaftliche Fakultät, 17487 Greifswald, (0 38 34) 8621 44, Fax (0 38 34) 8680077

663. Wallrabenstein, Dr. Astrid, Professorin, Prälat-Diehl-Str. 17, 64285 Darmstadt, (0 61 51) 651 09;

Goethe-Universität Frankfurt am Main, Fachbereich Rechtswissenschaften, Professur für Öffentliches Recht mit einem Schwerpunkt im Sozialrecht, Postfach 1119 32, 60054 Frankfurt am Main, (069) 798-34 288, Fax (069) 798-34 514

E-Mail professur-wallrabenstein@jura.uni-frankfurt.de 
664. Walter, Dr. Christian, Professor, Friedrich-List-Str. 98, 81377 München, (089) 787077 04;

Ludwig-Maximilians-Universität München, Institut für Internationales Recht, Lehrstuhl für Öffentliches Recht und Völkerrecht, Prof.-Huber-Platz 2, 80539 München, (089) 2180-2798, Fax (089) 2180-3841, E-Mail cwalter@jura.uni-muenchen.de

665. Weber, Dr. Albrecht, Professor, Weidenweg 20, 49143 Bissendorf, (0 54 02) 39 07;

Universität Osnabrück, 49069 Osnabrück, (05 41) 9 69-61 38,

E-Mail aweber@uos.de

666. Weber, Dr. Karl, o. Universitätsprofessor, Noldinstr. 14, A-6020 Innsbruck, (00 43) 06 64-1 6257 39; Universität Innsbruck, Institut für Öffentliches Recht, Finanzrecht und Politikwissenschaft, Innrain 80, A-6020 Innsbruck, (00 43) 5 12-5 07-82 30, E-Mail karl.weber@uibk.ac.at

667. Weber-Dürler, Dr. Beatrice, o. Professorin, Ackermannstr. 24, CH-8044 Zürich, (00 41) 4426204 20;

E-Mail beatrice.weber-duerler@rwi.uzh.ch

668. Wegener, Dr. Bernhard W., Professor, Friedrich-Alexander-Universität, Lehrstuhl für Öffentliches Recht, Schillerstraße 1, 91054 Erlangen, (0 91 31) 85292 85, Fax (0 91 31) 85264 39, E-Mail sekretariat.wegener@jura.uni-erlangen.de

669. Wehr, Dr. Matthias, Professor, Alter Kirchweg 24, 28717 Bremen, (04 21) 690800 25;

Hochschule für Öffentliche Verwaltung Bremen (HfÖV), Doventorscontrescarpe 172 C, 28195 Bremen, (04 21) 36119 617, E-Mail matthias.wehr@hfoev.bremen.de 
670. Weiß, Dr. Norman, Privatdozent, Martin-Luther-Str. 56, 10779 Berlin; MenschenRechtsZentrum der Universität Potsdam, August-Bebel-Str. 89, 14482 Potsdam, (03 31) 97734 50, Fax (03 31) 97734 51, E-Mail weiss@uni-potsdam.de

671. Wei $\beta$, Dr. Wolfgang, Universitätsprofessor, Deutsche Hochschule für Verwaltungswissenschaften Speyer, Lehrstuhl für Öffentliches Recht, Völker- und Europarecht, Freiherr-vom-Stein-Str. 2, 67346 Speyer, (0 62 32) 6 54-3 31, Fax (0 62 32) 6 54-1 23, E-Mailwweiss@brookes.ac.uk

672. Welti, Dr. Felix, Professor, Marquardplatz 3, 23554 Lübeck, (04 51) 81327 42, Fax (04 51) 81327 43;

Universität Kassel, FB 01 Humanwissenschaften, Institut für Sozialwesen, Abteilung Sozialpolitik, Recht und Soziologie, Arnold-Bode-Str. 10, 34109 Kassel, (0561) 8042 970, E-Mail mail@felix-welti.de

673. Wendt, Dr. Rudolf, o. Professor, Schulstr. 45, 66386 St. Ingbert-Hassel, (0 68 94) 532 87, Fax (0 68 94) 532 50;

Lehrstuhl für Staats- und Verwaltungsrecht, Wirtschafts-, Finanz- und Steuerrecht, Rechts- und Wirtschaftswissenschaftliche Fakultät, Universität des Saarlandes, Postfach 1511 50, 66041 Saarbrücken, (06 81) 3 02-21 04 oder -31 04, Fax (06 81) 302-47 79, E-Mail r.wendt@mx.uni-saarland.de

674. Wernsmann, Dr. Rainer, Professor, Johann-Bergler-Straße 8, 94032 Passau; Universität Passau, Lehrstuhl für Staats- und Verwaltungsrecht, insb. Finanz- und Steuerrecht, Innstr. 40, 94032 Passau, (08 51) 5 09-23 51, Fax (08 51) 5 09-23 52, E-Mail wernsmann@uni-passau.de 
675. Wessely, Dr. Wolfgang, Privatdozent, Universität Wien, Institut für Staats- und Verwaltungsrecht, Schottenbastei 10-16, A-1010 Wien, +43 (0)1 9005 11216, Fax +43 (0)1 9005 11210, E-Mail wolfgang.wessely@univie.ac.at

676. Wiederin, Dr. Ewald, Universitätsprofessor, Universität Wien, Institut für Staats- und Verwaltungsrecht, Schottenbastei 10-16, A-1010 Wien, (00 43) 14277354 82, Fax (00 43) 14277354 89, E-Mail ewald.wiederin@univie.ac.at

677. Wieland, Dr. Joachim, LL.M., Universitätsprofessor, Gregor-Mendel-Straße 13, 53115 Bonn, (02 28) 923993 34, Fax (02 28) 32948 98;

Lehrstuhl für öffentliches Recht, Finanz- und Steuerrecht, Deutsche Hochschule für Verwaltungswissenschaften Speyer, Postfach 1409, 67324 Speyer, (0 62 32) 6543 55, Fax (0 62 32) 6543 06, E-Mail wieland@dhv-speyer.de

678. Wielinger, Dr. Gerhard, Universitätsdozent, Bergmanngasse 22, A-8010 Graz, (00 43) 3 16-31 87 14, dienstl. (00 43) 3 16-70 312428

679. Wieser, DDr. Bernd, a.o. Universitätsprofessor, Wittenbauerstr. 76, A-8010 Graz; Institut für Öffentliches Recht, Politikwissenschaft und Verwaltungslehre, Karl-Franzens-Universität Graz, Universitätsstr. 15/C3, A-8010 Graz, (00 43) 3 16-3 80-33 81 oder -33 83, Fax (00 43) 3 16-3 80-94 50, E-Mail bernd.wieser@uni-graz.at

680. Wildhaber, Dr. Luzius, LL.M., J.S.D., Dres. h.c., LL.D.h.c., o. Professor, Auf der Wacht 21, CH-4104 Oberwil, (0 041) 6140125 21, E-Mail luzius.wildhaber@unibas.ch

681. Wilke, Dr. Dieter, Präsident des OVG Berlin a.D., Universitätsprofessor a.D., apl. Professor an der Freien Universität Berlin, Schweinfurthstr.10, 14195 Berlin 
682. Will, Dr. iur. Dr. phil. Martin, M.A., LL.M. (Cambr.), Professor, Hartmann-Ibach-Straße 105, 60389 Frankfurt, EBS Universität für Wirtschaft und Recht, Lehrstuhl für Staatsrecht, Verwaltungsrecht, Europarecht, Recht der neuen Technologien und Rechtsgeschichte, Gustav-Stresemann-Ring 3, 65189 Wiesbaden, (0611) 7102 2232, Fax (0611) 7102102232 , E-Mail martin.will@ebs.edu

683. Will, Dr. Rosemarie, Professorin, Humboldt-Universität zu Berlin, Juristische Fakultät, Unter den Linden 6, 10099 Berlin, (0 30) 209333 00-36 82, Fax (0 30) 209334 53, E-Mail Rosemarie.Will@rewi.hu-berlin.de

684. Wimmer, Dr. Norbert, o. Universitätsprofessor, Heiliggeiststr. 16, A-6020 Innsbruck, (00 43) 5 12-58 61 44;

Universität Innsbruck, Institut für Öffentliches Recht und Politikwissenschaften, Innrain 80/82, A-6020 Innsbruck, (00 43) 5 12-82 00 oder -82 01, E-Mail Veronika.Obojes@uibk.ac.at

685. Windoffer, Dr. Alexander, Privatdozent, Deutsches Forschungsinstitut für öffentliche Verwaltung Speyer, Freiherr-vom-Stein-Str. 2, 67346 Speyer, (06232) 654-399, Fax (06232) 654-290, E-Mail windoffer@foev-speyer.de

686. Windthorst, Dr. Kay, Professor, Prinzregentenstr. 75, 81675 München, (01 62) 90200 76;

Professur für Öffentliches Recht, Universität Bayreuth, Rechts- und Wirtschaftswissenschaftliche Fakultät, Universitätsstr. 30, Gebäude B 9, 95447 Bayreuth, (09 21) 55-35 19, Fax (09 21) 55-43 31, E-Mail kwindt@t-online.de 
687. Winkler, Dr. Dr. h.c. Günther, a.o. Universitätsprofessor, Reisnerstr. 22/5/11, A-1030 Wien, (00 43) 171344 15;

Universität Wien, Schottenbastei 10-16, A-1010 Wien, (00 43) $140103-3131$

688. Winkler, Dr. Markus, Privatdozent, Kaffeegasse 2 a, 65510 Idstein, E-Mail winkler.jura@web.de; Johannes-Gutenberg-Universität Mainz, Fachbereich Rechts- und Wirtschaftswissenschaften, 55099 Mainz, E-Mail mwinkl@uni-mainz.de; Hessisches Kultusministerium, Luisenplatz 10, 65185 Wiesbaden, (0611) 368 2517,

E-Mail markus.winkler@hkm.hessen.de

689. Winkler, Dr. Roland, a.o. Univ.-Prof., Borromäumstraße 10/2, A-5020 Salzburg, (00 43) 662641260 oder (00 43) 67690701 71; Fachbereich Öffentliches Recht, Universität Salzburg, Kapitelgasse 5-7, A-5020 Salzburg, (00 43) 662804436 24, Fax (00 43) 662804436 29, E-Mail roland.winkler@sbg.ac.at

690. Winter, Dr. Gerd, Professor,

FB 6: Rechtswissenschaft, Universität Bremen, Postfach 3304 40, 28334 Bremen, (04 21) 2 18-28 40, Fax (04 21) 2 18-34 94, E-Mail gwinter@uni-bremen.de

691. Winterhoff, Dr. Christian, apl. Prof., Theodor-Storm-Straße 1, 22869 Schenefeld, (0 40) 854003 76, Fax (0 40) 854148 80, E-Mail post@christianwinterhoff.de; Graf von Westphalen, Große Bleichen 21, 20354 Hamburg, (0 40) 35922 -2 64, Fax (0 40) 35922 -2 93, E-Mail christian.winterhoff@grafvonwestphalen.com 
692. Winzeler, Dr. Christoph, LL. M. (Harv.) Privatdozent, St.-Jakobs-Strasse 96, CH-4052 Basel, (00 41) 612959393 (Büro), Fax (00 41) 612725382 (Büro), E-Mail christoph.winzeler@span.ch;

Universität Fribourg, Institut für Religionsrecht, Miséricorde, Büro 4119, CH-1700 Fribourg, (00 41) 2630080 23, Fax (00 41) 2630096 66, E-Mail christoph.winzeler@sba.ch

693. Wißmann, Dr. Hinnerk, Professor, Universität Bayreuth, Lehrstuhl für Öffentliches Recht und Wirtschaftsrecht, 95440 Bayreuth, (09 21) 55-2876 oder -2875 (Sekr.), Fax (09 21) 55-58 23, E-Mail hinnerk.wissmann@uni-bayreuth.de

694. Wittinger, Dr. Michaela, Professorin, Schauinslandstraße 1, 76199 Karlsruhe, (07 21) 5916 81, E-Mail MichaelaWittinger@web.de; FH des Bundes für öffentliche Verwaltung, FB Bundeswehrverwaltung, Professur für Öffentliches Recht (insb. Staats- und Europarecht) Seckenheimer Landstraße 10, 68163 Mannheim, (06 21) 4295-4479, Fax (06 21) 4295-42222

695. Wittmann, Dr. Heinz, a.o. Universitätsprofessor, Steinböckengasse 4/14, A-1140 Wien, (00 43) 191431 75; Verlag Medien und Recht $\mathrm{GmbH}$, Danhausergasse 6, A-1040 Wien, (00 43) 150527 66, Fax (00 43)15 0527 66-15

696. Wittreck, Dr. Fabian, Professor, Cheruskerring 51, 48147 Münster, (02 51) 2006288 , Westfälische Wilhelms-Universität Münster, Professur für Öffentliches Recht, Bispinghof 25/25, 48143 Münster, (02 51) 83211 99, Fax (02 51) 83224 03, E-Mail fwitt_01@uni-muenster.de 
697. Wolf, Dr. Joachim, Professor, Von-Velsen-Straße 17, 44625 Herne, (0 23 23) 4596 25;

Juristische Fakultät, Ruhr-Universität Bochum, Umweltrecht, Verwaltungsrecht und Verwaltungslehre, Gebäude GC, Universitätsstr. 150, 44789 Bochum, (02 34) 3 22-52 52, Fax (02 34) 32144 21, E-Mail LS.Wolf@jura.ruhr-uni-bochum.de

698. Wolff, Dr. Heinrich Amadeus, Professor, Rudolf-Ditzen-Weg 12, 13156 Berlin, (0 30) 480979 48;

Lehrstuhl für Öffentliches Recht, insbesondere Staatsrecht und Verfassungsgeschichte, Europa-Universität Viadrina, Große Scharrnstr. 59, 15230 Frankfurt (Oder), (03 35) 553422 95, Fax (03 35) 553424 18, Mobil (01 63) 90124 45, E-Mail Heinrich.Wolff@euv-frankfurt-o.de

699. Wolfrum, Dr. Dr. h.c. Rüdiger, o. Professor, Mühltalstr. 129 b, 69121 Heidelberg, (0 62 21) 4752 36;

MPI für ausländisches öffentliches Recht und Völkerrecht, Im Neuenheimer Feld 535, 69120 Heidelberg, (0 62 21) 48 22-55 oder -56, Fax (0 62 21) 4822 88, E-Mail wolfrum@mpil.de

700. Wollenschläger, Dr. Ferdinand, Professor, Max-Planck-Str. 8, 81675 München, (089) 470279 73;

Universität Augsburg, Juristische Fakultät, Lehrstuhl für Öffentliches Recht, Europarecht und Öffentliches Wirtschaftsrecht, Universitätsstr. 24, 86135 Augsburg, (0821) 598-4551, Fax (0821) 598-4552, E-Mail ferdinand.wollenschlaeger@jura.uni-augsburg.de 
701. Wolter, Dr. Henner, Privatdozent, RA, Rechtsanwälte Hensche \& Wolter, Knesebeckstr. 76, 10623 Berlin

(0 30) 93933 30, Fax (0 30) 939333 33, E-Mail info@rawolter-berlin.de; Humboldt-Universität zu Berlin, Institut für Öffentliches Recht und Rechtsphilosophie, Unter den Linden 6, 10099 Berlin, (0 30) 20 93-34 72, Fax (0 30) 20 93-34 52

702. Würtenberger, Dr. Thomas, o. Professor, Beethovenstr. 9, 79100 Freiburg, (07 61) 786 23;

Universität Freiburg, Postfach, 79085 Freiburg (07 61) 2 03-22 46 oder -22 49, E-Mail Thomas.Wuertenberger@jura.uni-freiburg.de

703. Wyduckel, Dr. Dieter, Professor, Juristische Fakultät, TU Dresden, 01062 Dresden, (03 51) 46 33-73 22, Fax (03 51) 46 33-72 09, E-Mail wyduckel@jura.tu-dresden.de

704. Wyss, Dr. iur. Martin, Professor, Höheweg 62, CH-3097 Liebefeld, (00 41) 3197299 93, Fax (00 41) 3197299 91, E-Mail mpwyss@bluewin.ch;

Stellvertretender Chef Fachbereich II für Rechtsetzung, Bundesamt für Justiz, Bundeshaus West, CH-3003 Bern, (00 41) 3132275 75, Fax (00 41) 3132278 37, E-Mail martin.wyss@bj.admin.ch

705. Zacher, Dr. Dr. h.c. mult. Hans F., o. Professor, Starnberger Straße 47, 82343 Pöcking, (0 81 57) 13 84;

MPI für ausländisches und internationales Sozialrecht Amalienstr. 33, 80799 München, Postfach 3401 21, 80098 München, (0 89) 386 02-5 02, Fax (0 89) 386 02-5 90

706. Zeh, Dr. Wolfgang, Professor, Ministerialdirektor a.D., Marktstr. 10, 72359 Dotternhausen, E-Mail cowo.zeh@gmx.de 
707. Zezschwitz, Dr. Friedrich von, em. Universitätsprofessor, Petersweiher 47, 35394 Gießen, (06 41) 451 52;

Universität Gießen, 35390 Gießen, (06 41) 7025020

708. Ziegler, Dr. Andreas R., LL.M., Professor, Gründenstraße 66, CH-8247 Flurlingen; Universität Lausanne, Juristische Fakultät, BFSH 1, CH-1015 Lausanne, E-Mail andreas.ziegler@unil.ch

709. Ziekow, Dr. Jan, Universitätsprofessor, Gartenstraße 3, 67361 Freisbach, (0 63 44) 59 02, Fax (0 63 44) 59 02;

Deutsche Hochschule für Verwaltungswissenschaften Speyer, Postfach 14 09, 67324 Speyer, (0 62 32) 65 40,

E-Mail ziekow@dhv-speyer.de

710. Ziller, Dr. Jacques, Professor, Università degli Studi di Pavia, Dipartimento di Economia, Statistica e Diritto, Via Strada Nuova 65, I-27100 Pavia, (0039) (0) 382-98 44 37, Fax (0039) (0) 382-98 44 35,

E-Mail jacques.ziller@unipv.it

711. Zimmer, Dr. Gerhard, Professor, Waldschützpfad 9, 12589 Berlin, (0 30) 64895 90; Universität der Bundeswehr, 22043 Hamburg, (0 40) 65412771

712. Zimmermann, Dr. Andreas, LL.M (Harvard), Professor, Heimat 29, 14165 Berlin, E-Mail azimmerm@freenet.de; Universität Potsdam, Lehrstuhl für Öffentliches Recht, insbesondere Staatsrecht, Europa- und Völkerrecht sowie Europäisches Wirtschaftsrecht und Wirtschaftsvölkerrecht, August-Bebel-Str. 89, 14482 Potsdam, (03 31) 9 77-35 16, Fax (03 31) 9 77-32 24, E-Mail andreas.zimmermann@uni-potsdam.de 
713. Zippelius, Dr. Dr. h.c. Reinhold, o. Professor, Niendorfstr. 5, 91054 Erlangen, (0 91 31) 557 26;

Universität Erlangen-Nürnberg, 91054 Erlangen, (0 91 31) 852820

714. Zuleeg, Dr. Dr. h.c. Manfred, Professor, Kaiser-Sigmund-Str. 32, 60320 Frankfurt am Main, (0 69) 5643 93;

Institut für Öffentliches Recht, Völker- und Europarecht, Universität Frankfurt, Senckenberganlage 31, 60325 Frankfurt am Main, (0 69) 798223 82, Fax (0 69) 798287 50, E-Mail M.Zuleeg@jur.uni-frankfurt.de 